\title{
Exploring CH-Activation Pathways in Bifunctional \\ Zirconocene/Borane Systems
}

\author{
Michael Hill, Gerhard Erker, * Gerald Kehr, Roland Fröhlich, ${ }^{\#}$ Olga Kataeva
}

Organisch-Chemisches Institut Universität Münster, Corrensstrasse 40, D-48149 Münster, Germany, erker@uni-muenster.de

\section{Supporting Material}

Reaction of complex 2 with $\mathrm{N}$-methylbenzimidazole, preparation of the adduct 4a. m.p. $194^{\circ} \mathrm{C}$

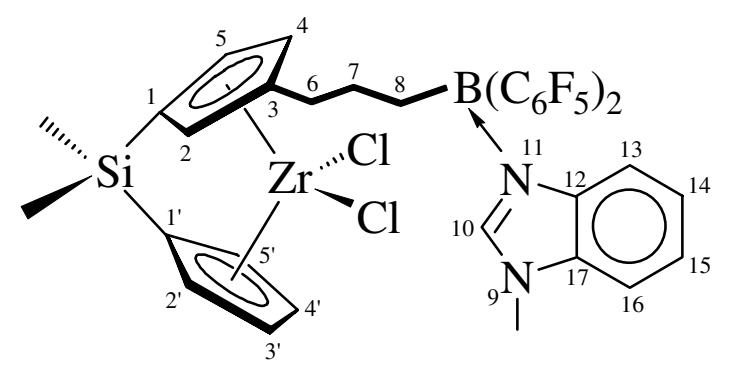
(DSC), $316^{\circ} \mathrm{C}$ (decomp.). IR (KBr): $\tilde{v}=2930(\mathrm{~m}), 1629$ (m), 1539 (m), 1503 (s), 1448 (s), 1313 (m), 1245 (s), $1189(\mathrm{w}), 1161(\mathrm{w}), 1084(\mathrm{~s}), 951(\mathrm{~m}), 791(\mathrm{~m}), 734(\mathrm{~m})$, $664(\mathrm{~m}), 448$ (w) $\mathrm{cm}^{-1}$. Anal. Calcd. for $\mathrm{C}_{35} \mathrm{H}_{27} \mathrm{~N}_{2} \mathrm{BF}_{10} \mathrm{Cl}_{2} \mathrm{SiZr}(866.6) \mathrm{C} 48.51, \mathrm{H} 3.14, \mathrm{~N} 3.23$;

found C 49.18, H 3.25, N 3.20\%. ${ }^{1} \mathrm{H}$ NMR (d -toluene, $\left.600 \mathrm{MHz}\right): \delta=8.27(\mathrm{~s}, 1 \mathrm{H}, 10-\mathrm{H}), 7.66(\mathrm{~m}, 1 \mathrm{H}$, 13-H), 6.81 (m, 2H, 14-H, 15-H), 6.72 (m, 1H, 3-H'), 6.67 (m, 1H, 4-H'), 6.47 (m, 1H, 16-H), 6.42 (m,

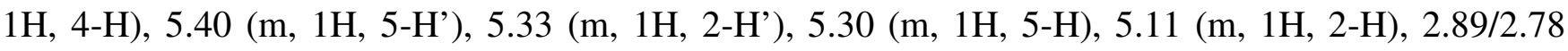
(each m, each 1H, 6-H, 6-H'), 2.76 (s, 3H, 9-H), 1.83/1.76 (each m, each 1H, 8-H, 8-H'), 1.55 (m, 2H, 
7-H), 0.13/0.07 (each s, each 3H, $\left.\mathrm{Si}\left(\mathrm{CH}_{3}\right)_{2}\right) .{ }^{13} \mathrm{C}\left\{{ }^{1} \mathrm{H}\right\}-\mathrm{NMR}\left(\mathrm{d}_{8}\right.$-toluene, $\left.150 \mathrm{MHz}\right): \delta=143.1(\mathrm{C} 3)$, 141.8 (C10), 136.6 (C12), 132.9 (C17), 128.7 (C4'), 128.3 (C4), 125.7 (C3'), 125.3 (C14), 125.2 (C15), 116.5 (C13), 114.4 (C2'), 114.3 (C2), 114.1 (C5), 112.8 (C5'), 111.0 (C16), 107.9 (C1'), 107.6 (C1), 33.0 (C6), 31.9 (C9), $26.2(\mathrm{C} 7), 23.8(\mathrm{C} 8),-5.7 /-6.5\left(\mathrm{Si}\left(\mathrm{CH}_{3}\right)_{2}\right) ; 148.8\left({ }^{1} \mathrm{~J}_{\mathrm{CF}}=238.1 \mathrm{~Hz}, \mathrm{o}-\mathrm{C}\right.$ of $\left.\mathrm{C}_{6} \mathrm{~F}_{5}\right), 139.5\left({ }^{1} \mathrm{~J}_{\mathrm{CF}}=250.9 \mathrm{~Hz}, \mathrm{p}-\mathrm{C}\right.$ of $\left.\mathrm{C}_{6} \mathrm{~F}_{5}\right), 137.5\left({ }^{1} \mathrm{~J}_{\mathrm{CF}}=249.8 \mathrm{~Hz}, \mathrm{~m}-\mathrm{C}\right.$ of $\left.\mathrm{C}_{6} \mathrm{~F}_{5}\right), 121.8$ (ipso-C of $\left.\mathrm{C}_{6} \mathrm{~F}_{5}\right)$. ${ }^{11} \mathrm{~B}\left\{{ }^{1} \mathrm{H}\right\}$ NMR $\left(\mathrm{d}_{8}\right.$-toluene, $\left.64 \mathrm{MHz}\right): \delta=-5.56\left(\mathrm{v}_{1 / 2}=324 \mathrm{~Hz}\right) .{ }^{19} \mathrm{~F}$ NMR $\left(\mathrm{d}_{8}\right.$-toluene, $\left.564 \mathrm{MHz}\right): \delta=-$ $132.2\left(\mathrm{~m}, 2 \mathrm{~F}, \mathrm{o}-\mathrm{F}\right.$ of $\left.\mathrm{C}_{6} \mathrm{~F}_{5}\right),-133.1\left(\mathrm{~m}, 2 \mathrm{~F}, \mathrm{o}-\mathrm{F}\right.$ ' of $\left.\mathrm{C}_{6} \mathrm{~F}_{5}\right),-158.8\left(\mathrm{t},{ }^{3} \mathrm{~J}_{\mathrm{FF}}=20.7 \mathrm{~Hz}, 1 \mathrm{~F}, \mathrm{p}-\mathrm{F}\right.$ of $\left.\mathrm{C}_{6} \mathrm{~F}_{5}\right),-$ $159.1\left(\mathrm{t},{ }^{3} \mathrm{~J}_{\mathrm{FF}}=20.8 \mathrm{~Hz}, 1 \mathrm{~F}, \mathrm{p}-\mathrm{F}^{\prime}\right.$ of $\left.\mathrm{C}_{6} \mathrm{~F}_{5}\right),-164.0\left(\mathrm{~m}, 2 \mathrm{~F}, \mathrm{~m}-\mathrm{F}\right.$ of $\left.\mathrm{C}_{6} \mathrm{~F}_{5}\right),-164.1\left(\mathrm{~m}, 2 \mathrm{~F}, \mathrm{~m}-\mathrm{F}^{\prime}\right.$ of $\left.\mathrm{C}_{6} \mathrm{~F}_{5}\right)$. 1D-TOCSY (d $\mathrm{d}_{8}$-toluene, $\left.600 / 600 \mathrm{MHz}\right): \delta\left({ }^{1} \mathrm{H}\right)_{\text {irr. }} / \delta\left({ }^{1} \mathrm{H}\right)_{\text {resp. }}=7.66 / 6.81,6.47(13-\mathrm{H} / 14-\mathrm{H}, 15-\mathrm{H}$, 16-H), 5.33 / 6.72, 6.67, 5.40 (2-H' / 3-H', 4-H', 5-H'), 5.11 / 6.42, 5.30 (2-H / 4-H, 5-H), 2.89 / 2.78 , 1.83, 1.76, $1.55\left(6-\mathrm{H} /\right.$ 6-H', 8-H, 8-H', 7-H). NOE-Diff (d $\mathrm{d}_{8}$-toluene, $\left.600 / 600 \mathrm{MHz}\right): \delta\left({ }^{1} \mathrm{H}\right)_{\text {irr. }} / \delta$ $\left({ }^{1} \mathrm{H}\right)_{\text {resp. }}=5.40$ / $6.67\left(5-\mathrm{H}^{\prime} / 4-\mathrm{H}^{\prime}\right), 0.13$ / 5.40, $5.30\left(\mathrm{Si}\left(\mathrm{CH}_{3}\right)_{2} / 5-\mathrm{H}^{\prime}, 5-\mathrm{H}\right), 0.07$ / 5.33, $5.11\left(\mathrm{Si}\left(\mathrm{CH}_{3}\right)_{2} /\right.$ 2-H', 2-H). GCOSY (d -toluene, $600 / 600 \mathrm{MHz}): \delta\left({ }^{1} \mathrm{H}\right) / \delta\left({ }^{1} \mathrm{H}\right)=8.27 / 2.76(10-\mathrm{H} / 9-\mathrm{H}), 7.66 /$ 6.81, 6.47 (13-H / 15-H, 14-H, 16-H), 6.72 / 6.67, 5.40, 5.33 (3-H' / 4-H', 5-H', 2-H'), 6.42 / 5.30, 5.11 (4-H / 5-H, 2-H), 2.89 / 2.78, 1.55 (6-H / 6-H', 7-H, 7-H'), 1.83 / 1.76, 1.55 (8-H / 8-H', 7-H). GHSQC $\left(\mathrm{d}_{8}\right.$-toluene, $\left.150 / 600 \mathrm{MHz}\right): \delta\left({ }^{13} \mathrm{C}\right) / \delta\left({ }^{1} \mathrm{H}\right)=141.8 / 8.27(\mathrm{C} 10 / 10-\mathrm{H}), 128.7 / 6.67(\mathrm{C} 4$ ' / 4-H'), 128.3 / 6.42 (C4 / 4-H), 125.7 / 6.72 (C3' / 3-H'), 125.2, 125.3 / 6.81 (C14, C15 / 14-H, 15-H), 116.5 / 7.66 (C13 / 13-H), 114.4 / 5.33 (C2' / 2-H'), 114.3 /5.11 (C2 / 2-H), 114.1 / 5.30 (C5 / 5-H), 112.8 / 5.40 (C5' / 5-H'), 111.0 / 6.47 (C16 / 16-H), 33.0 / 2.89, 2.78 (C6 / 6-H, 6-H'), 31.9 / 2.76 (C9 /9-H), 26.2 / $1.55(\mathrm{C} 7 / 7-\mathrm{H}), 23.3$ / 1.83, $1.76\left(\mathrm{C} 8\right.$ / 8-H, 8-H'), -5.7 / $0.13\left(\mathrm{Si}\left(\mathrm{CH}_{3}\right)_{2} / \mathrm{Si}\left(\mathrm{CH}_{3}\right)_{2}\right),-6.5$ / $0.07\left(\mathrm{Si}\left(\mathrm{CH}_{3}\right)_{2} / \mathrm{Si}\left(\mathrm{CH}_{3}\right)_{2}\right)$. GHMBC $\left(\mathrm{d}_{8}\right.$-toluene, $\left.150 / 600 \mathrm{MHz}\right): \delta\left({ }^{13} \mathrm{C}\right) / \delta\left({ }^{1} \mathrm{H}\right)=143.1 / 6.42,5.30$, 5.11, 2.89, 2.78 (C3 / 4-H, 5-H, 2-H, 6-H, 6-H'), 141.8 / 2.76 (C10 / 9-H), 136.6 / 8.27, 6.81, 6.47 (C12 / 10-H, 14-H, 15-H, 16-H), 132.9 / 8.27, 7.66, 6.81, 2.76 (C12 / 10-H, 13-H, 14-H, 15-H, 9-H), 128.3 / 5.11, 2.89, 2.78 (C4 / 2-H, 6-H, 6-H'), 125.3 / 6.47 (C14 / 16-H), 125.2 / 7.66 (C15 / 13-H), 116.5 / 6.81 (C13 / 15-H, 14-H), 114.3 / 6.42, 2.89 (C2 / 4-H, 6-H), 114.1 / 5.11 (C5 / 2-H), 112.8 / 
5.33 (C5' / 2-H'), 111.0 / 6.81 (C16 / 14-H, 15-H), 107.9 / 5.40, 5.33, 0.13, 0.07 (C1' / 5-H', 2-H', $\left.\mathrm{Si}\left(\mathrm{CH}_{3}\right)_{2}, \mathrm{Si}\left(\mathrm{CH}_{3}\right)_{2}\right), 107.6$ / 6.42, 5.30, 5.11, 0.13, 0.07 (C1 / 4-H, 5-H, 2-H, $\left.\mathrm{Si}\left(\mathrm{CH}_{3}\right)_{2}, \mathrm{Si}\left(\mathrm{CH}_{3}\right)_{2}\right), 33.0$ / 1.83, 1.76, 1.55 (C6 / 8-H, 7-H), 31.9 / 8.27 ( C9 / 10-H), 26.2 / 2.89, 2.78, 1.83, 1.76 (C7 / 6-H, 6-H', 8-H, 8-H'), -5.7 / $0.07\left(\mathrm{Si}\left(\mathrm{CH}_{3}\right)_{2} / \mathrm{Si}\left(\mathrm{CH}_{3}\right)_{2}\right),-6.5 / 0.13\left(\mathrm{Si}\left(\mathrm{CH}_{3}\right)_{2} / \mathrm{Si}\left(\mathrm{CH}_{3}\right)_{2}\right) .{ }^{19} \mathrm{~F} /{ }^{19} \mathrm{~F} \mathrm{GCOSY}\left(\mathrm{d}_{8}-\right.$ toluene, $564 / 564 \mathrm{MHz}): \delta\left({ }^{19} \mathrm{~F}\right) / \delta\left({ }^{19} \mathrm{~F}\right)=-132.2 /-164.0(\mathrm{o}-\mathrm{F} / \mathrm{m}-\mathrm{F}),-133.1 /-164.1\left(\mathrm{o}-\mathrm{F} ' / \mathrm{m}-\mathrm{F}^{\prime}\right),-$ 158.8 / -164.0 (p-F / m-F), -159.1 / -164.1 (p-F' / m-F'), -164.0 / -132.2, -158.8 (m-F / o-F, p-F), 164.1 / -133.1, -159.1 (m-F' / o-F', p-F').

Reaction of complex 2 with 1,2-dimethylbenzimidazole; preparation of $4 \mathrm{~b}$. m.p. $207^{\circ} \mathrm{C}$ (DSC).

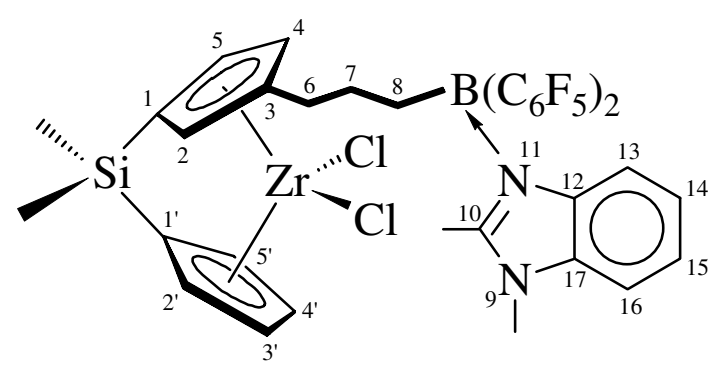
Anal. Calcd. for $\mathrm{C}_{36} \mathrm{H}_{29} \mathrm{~N}_{2} \mathrm{BF}_{10} \mathrm{Cl}_{2} \mathrm{SiZr}$ (880.6): C 49.10, H 3.32, N 3.18; found C 49.25, H 3.09, N 3.05\%. IR $(\mathrm{KBr}): \widetilde{v}=2909(\mathrm{w}), 1630(\mathrm{~m}), 1504(\mathrm{~s}), 1454(\mathrm{~s})$, 1161 (w), 1077 (s), 958 (s), 811 (s), 734 (s), 664 (m), $530(\mathrm{w}), 436(\mathrm{w}) \mathrm{cm}^{-1} .{ }^{1} \mathrm{H}$ NMR $\left(\mathrm{d}_{8}\right.$-toluene, $\left.600 \mathrm{MHz}\right)$ :

$\delta=7.43(\mathrm{~m}, 1 \mathrm{H}, 13-\mathrm{H}), 6.88(\mathrm{~m}, 1 \mathrm{H}, 15-\mathrm{H}), 6.85(\mathrm{~m}, 1 \mathrm{H}, 14-\mathrm{H}), 6.65\left(\mathrm{~m}, 1 \mathrm{H}, 3-\mathrm{H}^{\prime}\right), 6.62(\mathrm{~m}, 1 \mathrm{H}, 4-$ $\left.\mathrm{H}^{\prime}\right), 6.60(\mathrm{~m}, 2 \mathrm{H}, 16-\mathrm{H}), 6.20(\mathrm{~m}, 1 \mathrm{H}, 4-\mathrm{H}), 5.36\left(\mathrm{~m}, 1 \mathrm{H}, 5-\mathrm{H}^{\prime}\right), 5.25\left(\mathrm{~m}, 2 \mathrm{H}, 5-\mathrm{H}, 2-\mathrm{H}^{\prime}\right), 5.01(\mathrm{~m}, 1 \mathrm{H}$, 2-H), $2.73(\mathrm{~m}, 2 \mathrm{H}, 6-\mathrm{H}), 2.52(\mathrm{~s}, 3 \mathrm{H}, 9-\mathrm{H}), 2.09$ (s, 3H, $\left.\mathrm{Me}_{10}-\mathrm{H}\right), 1.95 / 1.62$ (each m, each 1H, 8-H, 8$\left.\mathrm{H}^{\prime}\right), 1.48 / 1.12$ (each m, each 1H, 7-H, 7-H'), 0.09/0.04 (each s, each 3H, $\left.\mathrm{Si}\left(\mathrm{CH}_{3}\right)_{2}\right) .{ }^{13} \mathrm{C}\left\{{ }^{1} \mathrm{H}\right\} \mathrm{NMR}\left(\mathrm{d}_{8}-\right.$ toluene, $150 \mathrm{MHz}): \delta=152.4(\mathrm{C} 10), 136.6(\mathrm{C} 12), 143.6(\mathrm{C} 3), 132.6$ (C17), 127.9 (C4'), 127.7 (C4), 126.3 (C3'), 124.1 (C14), 124.0 (C15), 116.9 (C13), 114.3 (C5), 114.2 (C2), 114.1 (C2'), 112.6 (C5'), 110.4 (C16), 107.8 (C1'), 107.5 (C1), 33.1 (C6), 29.2 (C9), 26.9 (C7), 23.4 (C8), $12.7\left(\mathrm{CMe}_{10}\right),-6.0 /-$ $6.9\left(\mathrm{Si}\left(\mathrm{CH}_{3}\right)_{2}\right) ; 148.8\left({ }^{1} \mathrm{~J}_{\mathrm{CF}}=241.3 \mathrm{~Hz}, \mathrm{o}-\mathrm{C}\right.$ of $\left.\mathrm{C}_{6} \mathrm{~F}_{5}\right), 148.5\left({ }^{1} \mathrm{~J}_{\mathrm{CF}}=240.0 \mathrm{~Hz}, \mathrm{o}-\mathrm{C}\right.$ of $\left.\mathrm{C}_{6} \mathrm{~F}_{5}\right), 139.9$ $\left({ }^{1} \mathrm{~J}_{\mathrm{CF}}=241.5 \mathrm{~Hz}, \mathrm{p}-\mathrm{C}\right.$ of $\left.\mathrm{C}_{6} \mathrm{~F}_{5}\right), 139.6\left({ }^{1} \mathrm{~J}_{\mathrm{CF}}=242.9 \mathrm{~Hz}, \mathrm{p}-\mathrm{C}\right.$ of $\left.\mathrm{C}_{6} \mathrm{~F}_{5}\right), 137.7\left({ }^{1} \mathrm{~J}_{\mathrm{CF}}=246.9 \mathrm{~Hz}, \mathrm{~m}-\mathrm{C}\right.$ of $\left.\mathrm{C}_{6} \mathrm{~F}_{5}\right) .{ }^{11} \mathrm{~B}\left\{{ }^{1} \mathrm{H}\right\}$ NMR (toluene- $\left.\mathrm{d}_{8}, 64 \mathrm{MHz}\right): \delta=-3.35\left(\mathrm{v}_{1 / 2}=491 \mathrm{~Hz}\right) .{ }^{19} \mathrm{~F}$ NMR (toluene- $\left.\mathrm{d}_{8}, 564 \mathrm{MHz}\right)$ : $\delta=-131.6\left(\mathrm{~m}, 2 \mathrm{~F}, \mathrm{o}-\mathrm{F}\right.$ of $\left.\mathrm{C}_{6} \mathrm{~F}_{5}\right),-131.7\left(\mathrm{~m}, 2 \mathrm{~F}, \mathrm{o}-\mathrm{F}^{\prime}\right.$ of $\left.\mathrm{C}_{6} \mathrm{~F}_{5}\right),-158.3\left(\mathrm{t},{ }^{3} \mathrm{~J}_{\mathrm{FF}}=20.6 \mathrm{~Hz}, 1 \mathrm{~F}, \mathrm{p}-\mathrm{F}^{\prime}\right.$ of 
$\left.\mathrm{C}_{6} \mathrm{~F}_{5}\right),-159.0\left(\mathrm{t},{ }^{3} \mathrm{~J}_{\mathrm{FF}}=20.8 \mathrm{~Hz}, 1 \mathrm{~F}, \mathrm{p}-\mathrm{F}\right.$ of $\left.\mathrm{C}_{6} \mathrm{~F}_{5}\right),-163.5\left(\mathrm{~m}, 2 \mathrm{~F}, \mathrm{~m}-\mathrm{F}\right.$ ' of $\left.\mathrm{C}_{6} \mathrm{~F}_{5}\right),-163.8(\mathrm{~m}, 2 \mathrm{~F}, \mathrm{~m}-\mathrm{F}$ of $\left.\mathrm{C}_{6} \mathrm{~F}_{5}\right) .1 \mathrm{D}-\mathrm{TOCSY}($ toluene-d $8,600 / 600 \mathrm{MHz}): \delta\left({ }^{1} \mathrm{H}\right)_{\text {irr. }} / \delta\left({ }^{1} \mathrm{H}\right)_{\text {resp. }}=7.43 / 6.88,6.85,6.60(13-\mathrm{H} / 15-$ H, 14-H, 16-H), 6.65 / 6.62, 5.36, 5.25 (3-H’ / 4-H', 5-H', 2-H'), 6.20 / 5.25, 5.01 (4-H / 5-H, 2-H), 2.73 / 1.95, 1.62, 1.48, 1.12 (6-H / 8-H, 8-H', 7-H, 7-H'). NOE-Diff (toluene-d $\left.{ }_{8}, 600 / 600 \mathrm{MHz}\right): \delta$ $\left({ }^{1} \mathrm{H}\right)_{\text {irr. }} / \delta\left({ }^{1} \mathrm{H}\right)_{\text {resp. }}=7.43$ / $6.85(13-\mathrm{H} / 14-\mathrm{H}), 6.60$ / 6.88, $2.52(16-\mathrm{H} / 15-\mathrm{H}, 9-\mathrm{H}), 6.65 / 5.25$ (3- $\mathrm{H}^{\prime} / 2-$ H'), 6.62 / 5.36 (4-H' / 5-H'), 6.20 / 5.25, 2.73, 1.48, 1.12 (4-H / 5-H, 6-H, 7-H, 7-H'), 5.36 / 6.62 , 0.09 (5-H' / 4-H', Si $\left.\left(\mathrm{CH}_{3}\right)_{2}\right), 5.01$ / 5.25, 2.73, 0.04 ( 2-H / 2-H', 6-H, 6-H', $\left.\mathrm{Si}\left(\mathrm{CH}_{3}\right)_{2}\right), 2.52$ / 6.60, 2.09

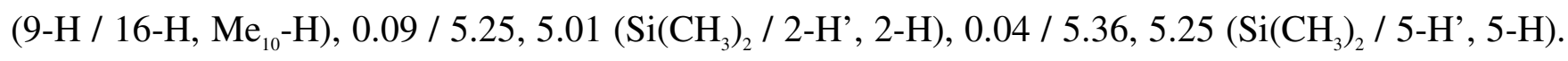
GCOSY (toluene-d $\left.{ }_{8}, 600 / 600 \mathrm{MHz}\right): \delta\left({ }^{1} \mathrm{H}\right) / \delta\left({ }^{1} \mathrm{H}\right)=7.43 / 6.85(13-\mathrm{H} / 14-\mathrm{H}), 6.88 / 6.60(15-\mathrm{H} /$ 16-H), 6.85 / 7.43 (14-H / 13-H), 6.60 / 6.88 (16-H / 15-H), 6.65 / 5.25 (3-H' / 2-H'), 6.62 / 5.36 (4-H' / 5-H'), 6.20 / 5.25, 5.01 (4-H / 5-H, 2-H), 5.36 / 6.65, 6.62, 5.25 (5-H’ / 3-H', 4-H', 2-H'), 5.25 / 6.65, 6.62, 5.36 (2-H' / 3-H', 4-H', 5-H'), 5.25 / 6.60, 6.62, 5.01 (5-H / 4-H, 2-H), 2.73 / 1.48, 1.12 (6-H / 7 H, 7-H'), 1.95 / 1.62, 1.48, 1.12 (8-H / 8-H', 7-H, 7-H'), 1.62 / 1.95, 1.48, 1.12 (8-H' / 8-H, 7-H, 7H'), 1.48 / 2.73, 1.621 .12 (7-H, 7-H' / 6-H, 8-H', 7-H'), 1.12 / 2.73, 1.95, 1.621 .48 (7-H' / 6-H, 8-H, 8-H', 7-H). GHSQC (toluene-d ${ }_{8}, 150$ / $\left.600 \mathrm{MHz}\right): \delta\left({ }^{13} \mathrm{C}\right) / \delta\left({ }^{1} \mathrm{H}\right)=127.9$ / 6.62 (C4' / 4-H'), 127.7 / 6.20 (C4 / 4-H), 126.3 / 6.65 (C3' / 3-H'), 124.1 / 6.85 (C14 / 14-H), 124.0 / 6.88 (C15 / 15-H), 116.9 / 7.43 (C13 / 13-H), 114.3 / 5.25 (C5 / 5-H), 114.2 / 5.01 (C2 / 2-H), 114.1 / 5.25 (C2' / 2-H'), 112.6 / 5.36 (C5' / 5-H'), 110.4 / 6.60 (C16 / 16-H), 33.1 / 2.73 (C6 / 6-H), 29.2 / 2.52 (C9 /9-H), 26.9 / 1.48, 1.12 (C7 / 7-H, 7-H'), 23.4 / 1.95, 1.62 (C8 / 8-H, 8-H'), 12.7 / $2.09\left(\mathrm{CMe}_{10} / \mathrm{Me}_{10}-\mathrm{H}\right),-6.0 / 0.09$ $\left(\mathrm{Si}\left(\mathrm{CH}_{3}\right)_{2} / \mathrm{Si}\left(\mathrm{CH}_{3}\right)_{2}\right),-6.9 / 0.04\left(\mathrm{Si}\left(\mathrm{CH}_{3}\right)_{2} / \mathrm{Si}\left(\mathrm{CH}_{3}\right)_{2}\right)$. GHMBC (toluene-d $\left.\mathrm{d}_{8}, 150 / 600 \mathrm{MHz}\right): \delta\left({ }^{13} \mathrm{C}\right) /$ $\delta\left({ }^{1} \mathrm{H}\right)=152.4 / 2.52,2.09\left(\mathrm{C} 10 / 9-\mathrm{H}, \mathrm{Me}_{10}-\mathrm{H}\right), 143.6 / 6.20,5.25,5.01,2.73(\mathrm{C} 3 / 4-\mathrm{H}, 5-\mathrm{H}, 2-\mathrm{H}, 6-$ H), 136.6 / 6.85, 6.60 (C12 / 14-H, 16-H), 132.6 / 7.43, 6.88, 2.52 (C17 / 13-H, 15-H, 9-H), 127.7 / 5.01, 2.73 (C4 / 2-H, 6-H, 6-H'), 126.3 / 5.36 (C3' / 5-H'), 124.1 / 6.60 (C14 / 16-H), 124.0 / 7.43 (C15 / 14-H), 116.9 / 6.88 (C13 / 15-H), 114.3 / 5.01, 6.20 (C5 / 2-H, 4-H), 114.2 / 6.20, 5.25, 2.73 (C2 / 4-H, 5-H, 6-H), 114.1 / 5.36 (C2' / 5-H'), 112.6 / 6.65, 5.25 (C5' / 3-H', 2-H'), 110.4 / 6.85 (C15 
/ 14-H), 107.5 / 6.20, 5.25, 5.01, 0.09, 0.04 (C1 / 4-H, 5-H, 2-H, $\left.\mathrm{Si}\left(\mathrm{CH}_{3}\right)_{2}, \mathrm{Si}\left(\mathrm{CH}_{3}\right)_{2}\right), 107.8$ / 6.65, 5.36, 5.25, 0.09, 0.04 (C1' / 3-H', 5-H', 2-H', $\left.\mathrm{Si}\left(\mathrm{CH}_{3}\right)_{2}, \mathrm{Si}\left(\mathrm{CH}_{3}\right)_{2}\right), 26.9$ / 2.73, 1.95, 1.62 (C7 / 6-H, 8-H, 8$\left.\mathrm{H}^{\prime}\right), 23.4$ / $2.73(\mathrm{C} 8 / 6-\mathrm{H}),-6.0 / 0.04\left(\mathrm{Si}\left(\mathrm{CH}_{3}\right)_{2} / \mathrm{Si}\left(\mathrm{CH}_{3}\right)_{2}\right),-6.9 / 0.09\left(\mathrm{Si}\left(\mathrm{CH}_{3}\right)_{2} / \mathrm{Si}\left(\mathrm{CH}_{3}\right)_{2}\right) .{ }^{19} \mathrm{~F} /{ }^{19} \mathrm{~F}$ GCOSY (toluene-d $\left.\mathrm{d}_{8}, 564 / 564 \mathrm{MHz}\right): \delta\left({ }^{19} \mathrm{~F}\right) / \delta\left({ }^{19} \mathrm{~F}\right)=-131.6 /-163.8(\mathrm{o}-\mathrm{F} / \mathrm{m}-\mathrm{F}),-131.7 /-163.5(\mathrm{o}-$ F' / m-F'), -158.3 / -163.5 (p-F' / m-F'), -159.0 / -163.8 (p-F / m-F), -163.5 / -131.7, -158.3 (m-F' / o-F', p-F'), -163.8 / - -131.6, -159.0 (m-F / o-F, p-F).

\section{Treatment of the adduct $4 \mathrm{a}$ with (trimethylsilyl)methyl lithium: synthesis of complex 5. mp}

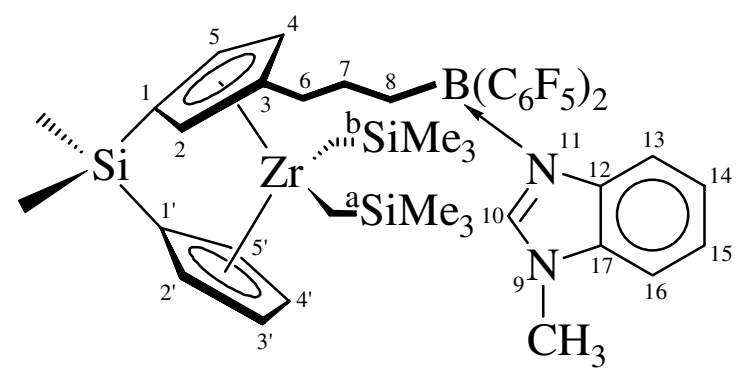
$119^{\circ} \mathrm{C}$. Anal. Calcd. for $\mathrm{C}_{43} \mathrm{H}_{49} \mathrm{~N}_{2} \mathrm{BF}_{10} \mathrm{Si}_{3} \mathrm{Zr}$ (970.1): C 53.24, H 5.09, N 2.89; found: C 54.06, H 4.79, N 2.73\%. IR (KBr): $\tilde{v}=3126(\mathrm{w}), 2916(\mathrm{w}), 1636(\mathrm{~m})$, $1546(\mathrm{~m}), 1504(\mathrm{~m}), 1440(\mathrm{~m}), 1252(\mathrm{~s}), 1189(\mathrm{~m}), 1091$ (s), 958 (s), $804(\mathrm{~s}), 742(\mathrm{~s}), 671(\mathrm{~s}), 553(\mathrm{w}), 420(\mathrm{w})$ $\mathrm{cm}^{-1} . \quad{ }^{1} \mathrm{H}$ NMR (dichloromethane-d $\left.\mathrm{d}_{2}, 600 \mathrm{MHz}\right): \delta=8.00(\mathrm{~s}, 1 \mathrm{H}, 10-\mathrm{H}), 7.62\left(\mathrm{~d},{ }^{3} \mathrm{~J}_{\mathrm{HH}}=8.4 \mathrm{~Hz}, 1 \mathrm{H}\right.$, 13-H), $7.55\left(\mathrm{~d},{ }^{3} \mathrm{~J}_{\mathrm{HH}}=8.2 \mathrm{~Hz}, 1 \mathrm{H}, 16-\mathrm{H}\right), 7.48(\mathrm{~m}, 1 \mathrm{H}, 15-\mathrm{H}), 7.36(\mathrm{~m}, 1 \mathrm{H}, 14-\mathrm{H}), 6.92\left(\mathrm{~m}, 1 \mathrm{H}, 3-\mathrm{H}^{\prime}\right)$, 6.91 (m, 1H, 4-H'), 6.69 (m, 1H, 4-H), 5.80 (m, 1H, 2-H'), 5.79 (m, 1H, 5-H'), 5.68 (m, 1H, 5-H), 5.60 (m, 1H, 2-H), 3.95 (s, 3H, 9-H), 2.69/2.66 (each m, each 1H, 6-H, 6-H'), 1.54 (m, 2H, 8-H), 1.37/1.31 (each m, each 1H, 7-H, 7-H'), 0.53/0.47 (each s, each 3H, $\left.\mathrm{Si}\left(\mathrm{CH}_{3}\right)_{2}\right), 0.01\left(\mathrm{~s}, 9 \mathrm{H},{ }^{\mathrm{a}} \mathrm{Si}\left(\mathrm{CH}_{3}\right)_{3}\right),-0.02(\mathrm{~s}$, $\left.9 \mathrm{H},{ }^{\mathrm{b}} \mathrm{Si}\left(\mathrm{CH}_{3}\right)_{3}\right), 0.05 /-0.10\left(\mathrm{AB},{ }^{2} \mathrm{~J}_{\mathrm{HH}}=5.8 \mathrm{~Hz}\right.$, each $\left.1 \mathrm{H},{ }^{\mathrm{b}} \mathrm{Si}\left(\mathrm{CH}_{2}\right)\right),-0.04 /-0.29\left(\mathrm{AB},{ }^{2} \mathrm{~J}_{\mathrm{HH}}=5.8 \mathrm{~Hz}\right.$, each $\left.1 \mathrm{H},{ }^{\mathrm{a}} \mathrm{Si}\left(\mathrm{CH}_{2}\right)\right) .{ }^{13} \mathrm{C}\left\{{ }^{1} \mathrm{H}\right\} \mathrm{NMR}$ (dichloromethane-d $\left.\mathrm{d}_{2}, 150 \mathrm{MHz}\right): \delta=131.4(\mathrm{C} 10), 126.3(\mathrm{C} 12), 125.3$ (C3), 123.5 (C17), 115.5 (C14, C15), 111.4 (C4), 110.0 (C3’), 106.6 (C13), 107.1 (C4'), 102.9 (C2), 102.6 (C2'), 101.5 (C16), 100.8 (C5'), 100.7 (C5), 91.9 (C1'), $91.4(\mathrm{C} 1), 38.4\left({ }^{\mathrm{a}} \mathrm{Si}\left(\mathrm{CH}_{2}\right)\right), 35.0$ $\left({ }^{\mathrm{b}} \mathrm{Si}\left(\mathrm{CH}_{2}\right)\right), 23.7(\mathrm{C} 9), 22.4(\mathrm{C} 6), 18.5(\mathrm{C} 7), 11.3(\mathrm{C} 8),-4.6 /-5.8\left(\mathrm{Si}\left(\mathrm{CH}_{3}\right)_{2}\right),-6.9\left({ }^{\mathrm{a}} \mathrm{Si}\left(\mathrm{CH}_{3}\right)_{3}\right),-7.1$ $\left({ }^{\mathrm{b}} \mathrm{Si}\left(\mathrm{CH}_{3}\right)_{3}\right) ; 138.2\left({ }^{1} \mathrm{~J}_{\mathrm{CF}}=238.8 \mathrm{~Hz}, \mathrm{o}-\mathrm{C}\right.$ of $\left.\mathrm{C}_{6} \mathrm{~F}_{5}\right), 129.1\left({ }^{1} \mathrm{~J}_{\mathrm{CF}}=251.5 \mathrm{~Hz}, \mathrm{p}-\mathrm{C}\right.$ of $\left.\mathrm{C}_{6} \mathrm{~F}_{5}\right), 127.1\left({ }^{1} \mathrm{~J}_{\mathrm{CF}}=\right.$ 249.7 Hz, m-C of $\left.\mathrm{C}_{6} \mathrm{~F}_{5}\right) .{ }^{11} \mathrm{~B}\left\{{ }^{1} \mathrm{H}\right\}$ NMR (dichloromethane-d $\left.\mathrm{d}_{2}, 64 \mathrm{MHz}\right): \delta=-5.16\left(\mathrm{v}_{1 / 2}=346 \mathrm{~Hz}\right) .{ }^{19} \mathrm{~F}-$ 
NMR (dichloromethane- $\left.\mathrm{d}_{2}, 564 \mathrm{MHz}\right): \delta=-133.3\left(\mathrm{~m}, 4 \mathrm{~F}, \mathrm{o}-\mathrm{F}\right.$ of $\left.\mathrm{C}_{6} \mathrm{~F}_{5}\right),-160.4\left(\mathrm{t},{ }^{3} \mathrm{~J}_{\mathrm{FF}}=20.2 \mathrm{~Hz}, 2 \mathrm{~F}, \mathrm{p}-\right.$

F of $\left.\mathrm{C}_{6} \mathrm{~F}_{5}\right),-165.1\left(\mathrm{~m}, 4 \mathrm{~F}, \mathrm{~m}-\mathrm{F}\right.$ of $\left.\mathrm{C}_{6} \mathrm{~F}_{5}\right) .1 \mathrm{D}-\mathrm{TOCSY}$ (dichloromethane- $\left.\mathrm{d}_{2}, 600 / 600 \mathrm{MHz}\right): \delta\left({ }^{1} \mathrm{H}\right)_{\text {irr. }} / \delta$ $\left({ }^{1} \mathrm{H}\right)_{\text {resp. }}=7.36$ / 7.62, 7.55, $7.48(14-\mathrm{H} / 13-\mathrm{H}, 16-\mathrm{H}, 15-\mathrm{H}), 6.92$ / 6.91, 5.80, $5.79\left(3-\mathrm{H}^{\prime} / 4-\mathrm{H}^{\prime}, 2-\mathrm{H}^{\prime}\right.$, 5-H'), 6.69/ 5.60, 5.68 (4-H / 2-H, 5-H), 2.69, 2.66 / 6.69, 5.68, 5.60, 1.54, 1.37, 1.31, (6-H, 6-H' / 4H, 5-H, 2-H, 8-H, 7-H, 7-H'). NOE-Diff (dichloromethane-d $2,600 / 600 \mathrm{MHz}): \delta\left({ }^{1} \mathrm{H}\right)_{\text {irr. }} / \delta\left({ }^{1} \mathrm{H}\right)_{\text {resp. }}=$

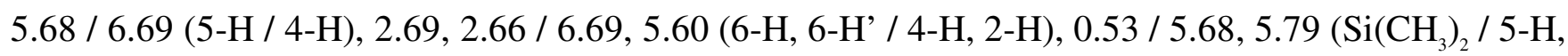

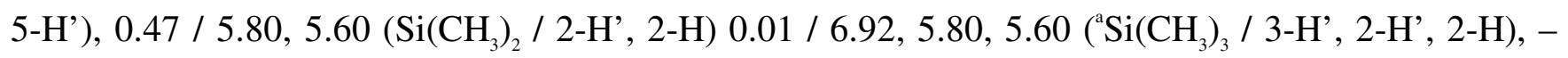
0.02 / 6.91, 6.69, 5.79, 5.68, ${ }^{\mathrm{b}} \mathrm{Si}\left(\mathrm{CH}_{3}\right)_{3} / 4-\mathrm{H}^{\prime}, 4-\mathrm{H}, 5-\mathrm{H}^{\prime}$, 5-H). GCOSY (dichloromethane- $\mathrm{d}_{2}, 600$ / 600 MHz): $\delta\left({ }^{1} \mathrm{H}\right) / \delta\left({ }^{1} \mathrm{H}\right)=8.00 / 3.95(10-\mathrm{H} / 9-\mathrm{H}), 7.62 / 7.36(13-\mathrm{H} / 14-\mathrm{H}), 7.55 / 7.48(16-\mathrm{H} / 15-\mathrm{H})$, 7.48 / 7.55, 7.36 (15-H / 16-H, 14-H), 7.36 / 7.62, 7.48 (14-H / 13-H, 15-H), 6.92, 6.91 / 5.80, 5.79 (3H', 4-H' / 2-H', 5-H'), 6.69 / 5.68, 5.60 (4-H / 5-H, 2-H), 2.69, 2.66 / 1.37, 1.31 (6-H, 6-H' / 7-H, 7H'), 1.54 / 1.37, 1.31 (8-H / 7-H, 7-H'), 1.37, 1.31 / 2.69, 2.66, 1.54 (7-H, 7-H' / 6-H, 6-H', 8-H). GHSQC (dichloromethane-d $\left.\mathrm{d}_{2}, 150 / 600 \mathrm{MHz}\right): \delta\left({ }^{13} \mathrm{C}\right) / \delta\left({ }^{1} \mathrm{H}\right)=131.4 / 8.00(\mathrm{C} 10 / 10-\mathrm{H}), 115.5 /$ 7.48, 7.36 (C14, C15 / 15-H, 14-H), 111.4 / 6.69 (C4 / 4-H), 110.0 / 6.92 (C3' / 3-H'), 107.1 / 6.91 (C4' / 4-H'), 106.6 / 7.62 (C13 / 13-H), 102.9 /5.60 (C2 / 2-H), 102.6 / 5.80 (C2' / 2-H'), 101.5 / 7.55, (C16 / 16-H), 100.8 / 5.79 (C5' / 5-H'), 100.7 / 5.68 (C5 / 5-H), 38.4 / -0.04, -0.29 ( ${ }^{\mathrm{a}} \mathrm{Si}\left(\mathrm{CH}_{2}\right)$ / $\left.{ }^{\mathrm{a}} \mathrm{Si}\left(\mathrm{CH}_{2}\right)\right), 35.0$ / 0.05, -0.10 ( $\left.{ }^{\mathrm{b}} \mathrm{Si}\left(\mathrm{CH}_{2}\right) /{ }^{\mathrm{b}} \mathrm{Si}\left(\mathrm{CH}_{2}\right)\right), 22.4$ / 2.69, 2.66 (C6 / 6-H, 6-H'), 23.7 / 3.95 (C9 19-H), 18.5 / 1.37, 1.31 (C7 / 7-H, 7-H'), 11.3 / 1.54 (C8 / 8-H), -4.6 / $0.53\left(\mathrm{Si}_{(}\left(\mathrm{CH}_{3}\right)_{2} / \mathrm{Si}\left(\mathrm{CH}_{3}\right)_{2}\right),-5.8$ / $0.47\left(\mathrm{Si}\left(\mathrm{CH}_{3}\right)_{2} / \mathrm{Si}\left(\mathrm{CH}_{3}\right)_{2}\right),-6.9 / 0.01\left({ }^{\mathrm{a}} \mathrm{Si}\left(\mathrm{CH}_{3}\right)_{3} /{ }^{\mathrm{a}} \mathrm{Si}\left(\mathrm{CH}_{3}\right)_{3}\right),-7.1 /-0.02\left({ }^{\mathrm{b}} \mathrm{Si}\left(\mathrm{CH}_{3}\right)_{3} /{ }^{\mathrm{b}} \mathrm{Si}\left(\mathrm{CH}_{3}\right)_{3}\right)$. GHMBC (dichloromethane- $\left.\mathrm{d}_{2}, 150 / 600 \mathrm{MHz}\right): \delta\left({ }^{13} \mathrm{C}\right) / \delta\left({ }^{1} \mathrm{H}\right)=131.4 / 3.95(\mathrm{C} 10 / 9-\mathrm{H}), 126.3 /$ 8.00, 7.55, 7.36 (C12 / 10-H, 16-H, 14-H), 125.3 / 6.69, 5.68, 5.60, 2.69, 2.66, 1.37, 1.31 (C3 / 4-H, 5H, 2-H, 6-H, 6-H', 7-H, 7-H'), 123.5 / 8.00, 7.62, 7.48, 3.95 (C12 / 10-H, 13-H, 15-H, 9-H), 111.4 / 5.68, 5.60, 2.69, 2.66 (C4 / 5-H, 2-H, 6-H, 6-H'), 110.0 / 6.91, 5.80 (C3' / 4-H', 2-H'), 107.1 / 6.92, 5.79 (C4' / 3-H', 5-H'), 106.6 / 7.48 (C13 / 15-H), 102.9 / 6.69, 5.68, 2.69, 2.66 (C2 / 4-H, 5-H, 6-H, 6-H'), 102.6 / 6.92, 6.91, 5.79 (C2' / 3-H', 4-H', 5-H'), 101.5 / 7.36 (C14 / 13-H), 100.8 / 6.92, 6.91, 
5.80 (C5' / 3-H', 4-H', 2-H'), 100.7 / 6.68, 5.60 (C5 / 4-H, 2-H), 91.9 / 6.92, 6.91, 5.80, 0.53, 0.47

(C1' / 3-H', 4-H', 2-H', $\left.\mathrm{Si}\left(\mathrm{CH}_{3}\right)_{2}, \mathrm{Si}\left(\mathrm{CH}_{3}\right)_{2}\right), 91.4$ / 6.69, 5.68, 5.60, 0.53, 0.47 (C1 / 4-H, 5-H, 2-H, $\left.\mathrm{Si}\left(\mathrm{CH}_{3}\right)_{2}, \mathrm{Si}\left(\mathrm{CH}_{3}\right)_{2}\right), 38.4 / 0.01\left({ }^{\mathrm{a}} \mathrm{Si}\left(\mathrm{CH}_{2}\right) /{ }^{\mathrm{a}} \mathrm{Si}\left(\mathrm{CH}_{3}\right)_{3}\right), 35.0 /-0.02\left({ }^{\mathrm{b}} \mathrm{Si}\left(\mathrm{CH}_{2}\right) /{ }^{\mathrm{b}} \mathrm{Si}\left(\mathrm{CH}_{3}\right)_{3}\right), 23.7 / 8.00$ (C9 / 10-H), 22.4 / 1.54, 1.37, 1.31 (C6 / 8-H, 7-H, 7-H'), 18.5 / 2.69, 2.66, 1.54 (C7 / 6-H, 6-H', 8-H), -4.6 / $0.47\left(\mathrm{Si}\left(\mathrm{CH}_{3}\right)_{2} / \mathrm{Si}\left(\mathrm{CH}_{3}\right)_{2}\right),-5.8 / 0.53\left(\mathrm{Si}\left(\mathrm{CH}_{3}\right)_{2} / \mathrm{Si}\left(\mathrm{CH}_{3}\right)_{2}\right),-6.9 /-0.29\left({ }^{\mathrm{a}} \mathrm{Si}\left(\mathrm{CH}_{3}\right)_{3} /{ }^{\mathrm{a}} \mathrm{Si}\left(\mathrm{CH}_{2}\right)\right),-$ $7.1 / 0.05,-0.11\left({ }^{\mathrm{b}} \mathrm{Si}\left(\mathrm{CH}_{3}\right)_{3} /{ }^{\mathrm{b}} \mathrm{Si}\left(\mathrm{CH}_{2}\right)\right)$.

Reaction of complex 4 a with LDA, formation of the products 7 and 7'. Diastereoisomer 7:

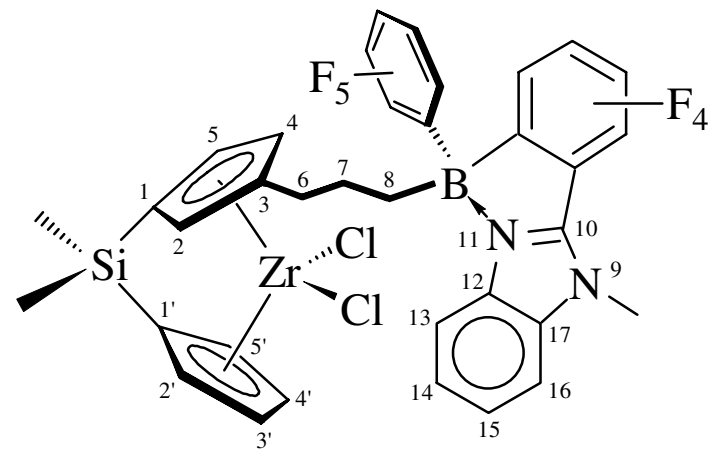

${ }^{1} \mathrm{H}$ NMR (tetrahydrofuran- $\left.\mathrm{d}_{8}, 600 \mathrm{MHz}\right): \delta=7.83(\mathrm{~m}, 1 \mathrm{H}$, 16-H), $7.48(\mathrm{~m}, 2 \mathrm{H}, 13-\mathrm{H}, 15-\mathrm{H}), 7.40(\mathrm{~m}, 1 \mathrm{H}, 14-\mathrm{H})$, $6.80\left(\mathrm{~m}, 1 \mathrm{H}, 3-\mathrm{H}^{\prime}\right), 6.79\left(\mathrm{~m}, 1 \mathrm{H}, 4-\mathrm{H}^{\prime}\right), 6.28(\mathrm{~m}, 1 \mathrm{H}, 4-$ H), $6.04\left(\mathrm{~m}, 1 \mathrm{H}, 5-\mathrm{H}^{\prime}\right), 5.89(\mathrm{~m}, 1 \mathrm{H}, 5-\mathrm{H}), 5.81(\mathrm{~m}, 1 \mathrm{H}$, 2-H'), 5.43 (m, 1H, 2-H), 4.34/4.32 (each d, each ${ }^{\mathrm{n}} \mathrm{J}_{\mathrm{HF}}=3.4 \mathrm{~Hz}$, each $\left.3 \mathrm{H}, 9-\mathrm{H}\right)^{*}, 2.42(\mathrm{~m}, 2 \mathrm{H}, 6-\mathrm{H})$,

1.56/1.37 (each m, each 1H, 8-H, 8-H'), 0.98/0.72 (each m, each 1H, 7-H, 7-H'), 0.69/0.60 (each s, each $\left.3 \mathrm{H}, \mathrm{Si}\left(\mathrm{CH}_{3}\right)_{2}\right) .{ }^{13} \mathrm{C}\left\{{ }^{1} \mathrm{H}\right\}$ NMR (tetrahydrofuran-d $\left.\mathrm{d}_{8}, 150 \mathrm{MHz}\right): \delta=152.5(\mathrm{C} 10), 143.3(\mathrm{C} 3), 138.1$ (C17), 133.6 (C12), 128.7 (C4’), 128.6 (C4), 126.8 (C3’), 126.1 (C14), 125.7 (C15), 115.2 (C2), 115.1 (C13), 115.0 (C5), 114.9 (C2'), 113.9 (C5'), 113.3 (C16), 108.7 (C1'), 108.1 (C1), 33.8 (C6), 33.0 (C9), $27.3(\mathrm{C} 7), 21.3(\mathrm{C} 8), \quad-5.5 /-5.7\left(\mathrm{Si}\left(\mathrm{CH}_{3}\right)_{2}\right)$. 1D-TOCSY (tetrahydrofuran-d $\left.\mathrm{d}_{8}, 600 / 600 \mathrm{MHz}\right): \delta$ $\left({ }^{1} \mathrm{H}\right)_{\text {irr. }} / \delta\left({ }^{1} \mathrm{H}\right)_{\text {resp. }}=7.48$ / 7.83, $7.40(13-\mathrm{H}, 15-\mathrm{H} / 16-\mathrm{H}, 14-\mathrm{H}), 5.81$ / 6.80, 6.79, 6.04 (2-H' / 3-H', 4H', 5-H'), 5.43 / 6.28, 5.89 (2-H / 4-H, 5-H), 2.42 / 1.56, 1.37, 0.98, 0.72 (6-H / 8-H, 8-H', 7-H, 7-H'). NOE-Diff (tetrahydrofuran-d $\left.{ }_{8}, 600 / 600 \mathrm{MHz}\right): \delta\left({ }^{1} \mathrm{H}\right)_{\text {irr. }} / \delta\left({ }^{1} \mathrm{H}\right)_{\text {resp. }}=6.80,6.79 / 6.04,5.81\left(3-\mathrm{H}^{\prime}, 4-\mathrm{H}^{\prime}\right.$ / 5-H', 2-H'), 6.28 / 5.89 (4-H / 5-H), 6.04 / 6.79, 5.89, 0.69 (5-H' / 4-H', 5-H, Si(CH$\left.)_{2}\right), 5.89$ / 6.28 , 6.04, 0.69 (5-H / 4-H, 5-H', $\left.\mathrm{Si}\left(\mathrm{CH}_{3}\right)_{2}\right), 5.81$ / 6.80, 5.43, 0.60 (2-H' / 3-H', 2-H, Si( $\left.\left.\mathrm{CH}_{3}\right)_{2}\right), 5.43$ / 5.81 , 2.42, 0.72, 0.60 (2-H / 2-H', 6-H, 7-H', $\left.\mathrm{Si}\left(\mathrm{CH}_{3}\right)_{2}\right), 0.69$ / 6.04, $5.89\left(\mathrm{Si}\left(\mathrm{CH}_{3}\right)_{2} /\right.$ 5-H', 5-H), 0.60 / 5.81, 
$5.43\left(\mathrm{Si}\left(\mathrm{CH}_{3}\right)_{2} / 2-\mathrm{H}^{\prime}, 2-\mathrm{H}\right)$. GCOSY (tetrahydrofuran-d $\left.{ }_{8}, 600 / 600 \mathrm{MHz}\right): \delta\left({ }^{1} \mathrm{H}\right) / \delta\left({ }^{1} \mathrm{H}\right)=7.83 / 7.48$

(16-H / 15-H), 7.48 / 7.83, 7.40 (13-H, 15-H / 16-H, 14-H), 7.40 / 7.48 (14-H / 13-H, 15-H), 6.80 / 6.04, 5.81 (3-H' / 5-H', 2-H’), 6.28 / 5.89, 5.43 (4-H / 5-H, 2-H), 6.04 / 6.80, 6.79, 5.81 (5-H' / 3-H', 4-H’, 2-H’), 5.89 / 6.28, 5.43 (5-H / 4-H, 2-H), 5.81 / 6.80, 6.79, 6.04 (2-H’ / 3-H', 4-H’, 5-H'), 5.43 / 6.28, 5.89 (2-H / 4-H, 5-H), 2.42 / 0.98, 0.72 (6-H / 7-H, 7-H'), 1.56 / 1.37, 0.98, 0.72 (8-H / 8-H', 7H, 7-H'), 1.37 / 1.56, 0.98, 0.72 (8-H' / 8-H, 7-H, 7-H'), 0.98 / 2.42, 1.56, 1.37, 0.72 (7-H / 6-H, 8-H, 8-H', 7-H'), 0.72 / 1.56, 0.98 (7-H' / 8-H, 7-H). GHSQC (tetrahydrofuran-d $\mathrm{d}_{8}, 150$ / $\left.600 \mathrm{MHz}\right): \delta\left({ }^{13} \mathrm{C}\right) /$ $\delta\left({ }^{1} \mathrm{H}\right)=128.7$ / $6.79\left(\mathrm{C}^{\prime} '\right.$ / 4-H'), 128.6 / 6.28 (C4 / 4-H), 126.8 / $6.80\left(\mathrm{C}^{\prime}\right.$ / 3-H'), 125.7/ 7.48 (C15 / 15-H), 126.1 / 7.40 (C14 / 14-H), 115.2 /5.43 (C2 / 2-H), 115.1 / 7.48 (C13 / 13-H), 115.0 / 5.89 (C5 / 5-H), 114.9 / 5.81 (C2' / 2-H'), 113.9 / 6.04 (C5' / 5-H'), 113.3 / 7.83 (C16 / 16-H), 33.8 / 2.42 (C6 / 6-H), 33.0 / 4.33 (C9 /9-H), 27.3 / 0.98, 0.72 (C7 / 7-H, 7-H'), 21.3 / 1.56, 1.37 (C8 / 8-H, 8-H'), -5.5 / $0.69\left(\mathrm{Si}\left(\mathrm{CH}_{3}\right)_{2} / \mathrm{Si}\left(\mathrm{CH}_{3}\right)_{2}\right),-5.7 / 0.60\left(\mathrm{Si}\left(\mathrm{CH}_{3}\right)_{2} / \mathrm{Si}\left(\mathrm{CH}_{3}\right)_{2}\right)$. GHMBC (tetrahydrofuran-d $\mathrm{d}_{8}, 150 / 600$ $\mathrm{MHz}): \delta\left({ }^{13} \mathrm{C}\right) / \delta\left({ }^{1} \mathrm{H}\right)=152.5 / 4.33(\mathrm{C} 10 / 9-\mathrm{H}), 143.3 / 6.28,5.89,5.43,2.42(\mathrm{C} 3 / 4-\mathrm{H}, 5-\mathrm{H}, 2-\mathrm{H}, 6-$ H), 138.1 / 7.48, 4.33 (C12 / 13-H, 15-H, 9-H), 133.6 / 7.83, 7.40 (C12 / 16-H, 14-H), 128.7 / 6.04 (C4' / 5-H'), 128.6 / 5.43, 2.42 (C4 / 2-H, 6-H), 126.1 / 7.83 (C14 / 16-H), 125.7 / 7.48 (C15 / 14-H), 115.2 / 6.28, 5.89, 2.42 (C2 / 4-H, 5-H, 6-H), 115.1 / 7.48 (C13 / 15-H), 115.0 / 5.43 (C5 / 2-H), 114.9 / 6.04 (C2' / 5-H'), 113.3 / 7.40 (C16 / 14-H), 108.7 / 6.04, 5.81, 0.69, 0.60 (C1' / 5-H', 2-H', $\left.\mathrm{Si}\left(\mathrm{CH}_{3}\right)_{2}, \mathrm{Si}\left(\mathrm{CH}_{3}\right)_{2}\right), 108.1 / 6.28,5.89,5.43,0.69,0.60\left(\mathrm{C} 1 / 4-\mathrm{H}, 5-\mathrm{H}, 2-\mathrm{H}, \mathrm{Si}\left(\mathrm{CH}_{3}\right)_{2}, \mathrm{Si}\left(\mathrm{CH}_{3}\right)_{2}\right), 33.8$ / 1.56 (C6 / 8-H), 33.0 / 8.27 ( C9 / 10-H), 27.3 / 2.42, 1.56, 1.37 (C7 / 6-H, 8-H, 8-H'), -5.5 / 0.60 $\left(\mathrm{Si}\left(\mathrm{CH}_{3}\right)_{2} / \mathrm{Si}\left(\mathrm{CH}_{3}\right)_{2}\right),-5.7 / 0.69\left(\mathrm{Si}\left(\mathrm{CH}_{3}\right)_{2} / \mathrm{Si}\left(\mathrm{CH}_{3}\right)_{2}\right)$. Diastereoisomer 7': ${ }^{1} \mathrm{H}-\mathrm{NMR}$ (tetrahydrofuran$\left.\mathrm{d}_{8}, 600 \mathrm{MHz}\right): \delta=7.83(\mathrm{~m}, 1 \mathrm{H}, 16-\mathrm{H}), 7.48(\mathrm{~m}, 2 \mathrm{H}, 13-\mathrm{H}, 15-\mathrm{H}), 7.40(\mathrm{~m}, 1 \mathrm{H}, 14-\mathrm{H}), 6.85(\mathrm{~m}, 1 \mathrm{H}, 3-$ H'), 6.79 (m, 1H, 4-H'), 6.21 (m, 1H, 4-H), 6.04 (m, 1H, 5-H'), 5.86 (m, 1H, 5-H), 5.84 (m, 1H, 2-H'), $5.47(\mathrm{~m}, 1 \mathrm{H}, 2-\mathrm{H}), 4.34 / 4.32\left(\right.$ each d, each ${ }^{\mathrm{n}} \mathrm{J}_{\mathrm{HF}}=3.4 \mathrm{~Hz}$, each 3H, 9-H)*, 2.47/2.38 (m, 2H, 6-H, 6-H'), $1.45(\mathrm{~m}, 2 \mathrm{H}, 8-\mathrm{H}), 0.93 / 0.83$ (each m, each 1H, 7-H, 7-H'), 0.69/0.62 (each s, each 3H, $\left.\mathrm{Si}\left(\mathrm{CH}_{3}\right)_{2}\right)$. ${ }^{13} \mathrm{C}\left\{{ }^{1} \mathrm{H}\right\}$ NMR (tetrahydrofuran-d $\left.\mathrm{d}_{8}, 150 \mathrm{MHz}\right): \delta=152.5(\mathrm{C} 10), 143.3(\mathrm{C} 3), 138.1(\mathrm{C} 17), 133.6(\mathrm{C} 12)$, 
128.7 (C4'), 128.5 (C4), 127.0 (C3’), 126.1 (C14), 125.7 (C15), 115.2 (C5), 115.1 (C2), 115.1 (C13), 114.8 (C2'), 113.9 (C5'), 113.3 (C16), 108.7 (C1'), 108.0 (C1), 33.8 (C6), 33.0 (C9), 27.1 (C7), 21.1 (C8), -5.5/-5.7 $\left(\mathrm{Si}\left(\mathrm{CH}_{3}\right)_{2}\right)$. 1D-TOCSY (tetrahydrofuran-d $\left.{ }_{8}, 600 / 600 \mathrm{MHz}\right): \delta\left({ }^{1} \mathrm{H}\right)_{\text {irr. }} / \delta\left({ }^{1} \mathrm{H}\right)_{\text {resp. }}=7.48$ / 7.83, 7.40, (13-H, 15-H / 16-H, 14-H), 5.84 / 6.85, 6.79, 6.04 (2-H' / 3-H', 4-H', 5-H'), 5.47 / 6.21, 5.86 (2-H / 4-H, 5-H), 2.47, 2.38 / 1.45, 0.93, 0.83 (6-H / 8-H, 7-H, 7-H'). NOE-Diff (tetrahydrofuran$\mathrm{d}_{8}, 600$ / $\left.600 \mathrm{MHz}\right): \delta\left({ }^{1} \mathrm{H}\right)_{\text {irr. }} / \delta\left({ }^{1} \mathrm{H}\right)_{\text {resp. }}=6.79$ / $6.04\left(4-\mathrm{H}^{\prime} / 5-\mathrm{H}^{\prime}\right), 6.21 / 5.86(4-\mathrm{H} / 5-\mathrm{H}), 6.04$ / 6.79, 5.86, 0.69 (5-H' / 4-H', 5-H, $\left.\mathrm{Si}\left(\mathrm{CH}_{3}\right)_{2}\right), 5.86$ / 6.21, 0.69 (5-H / 4-H, $\left.\mathrm{Si}\left(\mathrm{CH}_{3}\right)_{2}\right), 5.84$ / 6.85, 5.47, 0.62 (2-H' / 3-H', 2-H, Si( $\left.\left(\mathrm{CH}_{3}\right)_{2}\right)$, 5.47 / 5.84, 2.47, 2.38, 0.83, 0.62 (2-H / 2-H', 6-H, 6-H', 7-H', Si $\left.\left(\mathrm{CH}_{3}\right)_{2}\right)$, 0.69 / 6.04, $5.86\left(\mathrm{Si}\left(\mathrm{CH}_{3}\right)_{2} /\right.$ 5-H', 5-H), 0.62 / 5.84, $5.47\left(\mathrm{Si}\left(\mathrm{CH}_{3}\right)_{2} /\right.$ 2-H', 2-H). GCOSY (tetrahydrofuran-d $\left.\mathrm{d}_{8}, 600 / 600 \mathrm{MHz}\right): \delta\left({ }^{1} \mathrm{H}\right) / \delta\left({ }^{1} \mathrm{H}\right)=7.83 / 7.48(16-\mathrm{H} / 15-\mathrm{H}), 7.48 / 7.83,7.40(13-$ H, 15-H / 16-H, 14-H), 7.40 / 7.48 (14-H / 13-H, 15-H), 6.85 / 5.84 (3-H’ / 2-H'), 6.79 / 6.85, 6.04 (4H' / 3-H', 5-H'), 6.21 / 5.86, 5.47 (4-H / 5-H, 2-H), 6.04 / 6.85, 6.79, 5.84 (5-H' / 3-H', 4-H', 2-H'), 5.86 / 6.21, 5.47 (5-H / 4-H, 2-H), 5.84 / 6.85, 6.04 (2-H’ / 3-H', 5-H’), 5.47 / 6.21, 5.86 (2-H / 4-H, 5H), 2.47 / 2.38, 0.93, 0.83 (6-H / 6-H', 7-H, 7-H'), 2.38 / 2.47, 0.93, 0.83 (6-H’/ 6-H, 7-H, 7-H'), 1.45 / 0.93, 0.83 (8-H / 7-H, 7-H'), 0.93 / 2.47, 2.38, 1.45, 0.83 (7-H / 6-H, 6-H', 8-H, 7-H'), 0.83 / 2.47, 2.38, 1.45, 0.93 (7-H' / 6-H, 6-H', 8-H, 7-H). GHSQC (tetrahydrofuran-d, $150 / 600 \mathrm{MHz}): \delta\left({ }^{13} \mathrm{C}\right) / \delta$ $\left({ }^{1} \mathrm{H}\right)=128.7$ / 6.79 (C4' / 4-H'), 128.5 / $6.21(\mathrm{C} 4$ / 4-H), 127.0 / 6.85 (C3' / 3-H'), $126.1 / 7.40$ (C14 / 14-H), $125.7 / 7.48$ (C15 / 15-H), 115.2 / 5.86 (C5 / 5-H), 115.1 /5.47 (C2 / 2-H), $115.1 / 7.48$ (C13 / 13-H), 114.8 / 5.84 (C2' / 2-H'), 113.9 / 6.04 (C5' / 5-H'), 113.3 / 7.83 (C16 / 16-H), 33.8 / 2.47, 2.38 (C6 / 6-H, 6-H'), 33.0 / 4.33 (C9 /9-H), 27.1 / 0.93, 0.83 (C7 / 7-H, 7-H'), 21.1 / 1.45 (C8 / 8-H), -5.5 / $0.69\left(\mathrm{Si}\left(\mathrm{CH}_{3}\right)_{2} / \mathrm{Si}\left(\mathrm{CH}_{3}\right)_{2}\right),-5.7 / 0.62\left(\mathrm{Si}\left(\mathrm{CH}_{3}\right)_{2} / \mathrm{Si}\left(\mathrm{CH}_{3}\right)_{2}\right)$. GHMBC (tetrahydrofuran-d $\mathrm{d}_{8}, 150 / 600$ $\mathrm{MHz}): \delta\left({ }^{13} \mathrm{C}\right) / \delta\left({ }^{1} \mathrm{H}\right)=152.5 / 4.33(\mathrm{C} 10 / 9-\mathrm{H}), 143.3 / 6.21,5.47,2.47,2.38(\mathrm{C} 3 / 4-\mathrm{H}, 2-\mathrm{H}, 6-\mathrm{H}, 6-$ H'), 138.1 / 7.48, 4.33 (C12 / 13-H, 15-H, 9-H), 133.6 / 7.83, 7.40 (C12 / 16-H, 14-H), 128.5 / 5.47, 2.47, 2.38 (C4 / 2-H, 6-H, 6-H'), 126.1 / 7.83 (C14 / 16-H), 125.7 / 7.48 (C15 / 13-H), 115.2 / 5.47 (C5 / 2-H), 115.1 / 6.21, 2.47, 2.38 (C2 / 4-H, 6-H ,6-H'), 115.1 / 7.48 (C13 / 15-H), 114.8 / 6.04 (C2' / 5- 
H'), 113.3 / 7.40 (C16 / 14-H), 108.7 / 6.04, 5.84, 0.69, 0.62 (C1' / 5-H', 2-H', $\left.\mathrm{Si}\left(\mathrm{CH}_{3}\right)_{2}, \mathrm{Si}\left(\mathrm{CH}_{3}\right)_{2}\right)$, 108.0 / 6.21, 5.86, 5.47, 0.69, $0.62\left(\mathrm{C} 1 / 4-\mathrm{H}, 5-\mathrm{H}, 2-\mathrm{H}, \mathrm{Si}\left(\mathrm{CH}_{3}\right)_{2}, \mathrm{Si}\left(\mathrm{CH}_{3}\right)_{2}\right), 33.8$ / 1.45, 0.930 .83 (C6 / 8-H, 7-H, 7-H'), 33.0 / 8.27 (C9 / 10-H), 27.1 / 2.47, 2.38, 1.45 (C7 / 6-H, 6-H', 8-H), -5.5 / 0.62 $\left(\mathrm{Si}\left(\mathrm{CH}_{3}\right)_{2} / \mathrm{Si}\left(\mathrm{CH}_{3}\right)_{2}\right),-5.7 / 0.69\left(\mathrm{Si}_{(}\left(\mathrm{CH}_{3}\right)_{2} / \mathrm{Si}\left(\mathrm{CH}_{3}\right)_{2}\right)$. The ${ }^{1} \mathrm{H}$ NMR of $9-\mathrm{H},{ }^{13} \mathrm{C}$ NMR of $\mathrm{B}\left(\mathrm{C}_{6} \mathrm{~F}_{5}\right)_{2},{ }^{11} \mathrm{~B}$ NMR and ${ }^{19} \mathrm{~F}$ NMR resonances could not be assigned specifically to the single isomers. ${ }^{1} \mathrm{H}$ NMR $\left(\mathrm{d}_{8}-\right.$ tetrahydrofuran, $600 \mathrm{MHz}, 300 \mathrm{~K}$ ): $\delta 4.34 / 4.32$ (each d, each ${ }^{\mathrm{n}} \mathrm{J}_{\mathrm{HF}}=3.4 \mathrm{~Hz}$, each $3 \mathrm{H}, 9-\mathrm{H}$ ). ${ }^{13} \mathrm{C}\left\{{ }^{1} \mathrm{H}\right\}$ NMR (d $\mathrm{d}_{8}$-tetrahydrofuran, $\left.150 \mathrm{MHz}, 300 \mathrm{~K}\right): \delta 149.9\left({ }^{1} \mathrm{~J}_{\mathrm{CF}}=243.7 \mathrm{~Hz}, \quad\right.$ o-C of $\left.\mathrm{C}_{6} \mathrm{~F}_{5}\right), 140.2$ $\left({ }^{1} \mathrm{~J}_{\mathrm{CF}}=246.8 \mathrm{~Hz}, \mathrm{p}-\mathrm{C}\right.$ of $\left.\mathrm{C}_{6} \mathrm{~F}_{5}\right), 137.8\left({ }^{1} \mathrm{~J}_{\mathrm{CF}}=243.7 \mathrm{~Hz}, \mathrm{~m}-\mathrm{C}\right.$ of $\left.\mathrm{C}_{6} \mathrm{~F}_{5}\right)$ (ipso-C of $\mathrm{C}_{6} \mathrm{~F}_{5}$ was not observed; no assignment of the annulated $\mathrm{C}_{6} \mathrm{~F}_{4}$-ring). ${ }^{11} \mathrm{~B}\left\{{ }^{1} \mathrm{H}\right\} \mathrm{NMR}$ (d $\mathrm{d}_{8}$-tetrahydrofuran, $64 \mathrm{MHz}, 298 \mathrm{~K}$ ): $\delta=-$ $4.40\left(v_{1 / 2}=274 \mathrm{~Hz}\right) .{ }^{19} \mathrm{~F}$ NMR $\left(\mathrm{d}_{8}\right.$-tetrahydrofuran, $\left.564 \mathrm{MHz}, 300 \mathrm{~K}\right): \delta-132.6,-135.8,-151.7,-159.3$ (each m, each 1F, 2-, 5-, 4-, 3-F of $\left.\mathrm{C}_{6} \mathrm{~F}_{4}\right),-133.5\left(\mathrm{~m}, 2 \mathrm{~F}\right.$, o-F of $\left.\mathrm{C}_{6} \mathrm{~F}_{5}\right),-134.2\left(\mathrm{~m}, 2 \mathrm{~F}, \mathrm{o}-\mathrm{F}\right.$ ' of $\left.\mathrm{C}_{6} \mathrm{~F}_{5}\right)$, $160.2\left(\mathrm{t},{ }^{3} \mathrm{~J}_{\mathrm{FF}}=20.1 \mathrm{~Hz}, 1 \mathrm{~F}, \mathrm{p}-\mathrm{F}\right.$ of $\left.\mathrm{C}_{6} \mathrm{~F}_{5}\right),-161.1\left(\mathrm{t},{ }^{3} \mathrm{~J}_{\mathrm{FF}}=20.0 \mathrm{~Hz}, 1 \mathrm{~F}, \mathrm{p}-\mathrm{F}{ }^{\prime}\right.$ of $\left.\mathrm{C}_{6} \mathrm{~F}_{5}\right),-165.5(\mathrm{~m}, 2 \mathrm{~F}, \mathrm{~m}-\mathrm{F}$ of $\left.\mathrm{C}_{6} \mathrm{~F}_{5}\right),-165.9\left(\mathrm{~m}, 2 \mathrm{~F}, \mathrm{~m}-\mathrm{F}\right.$ ' of $\left.\mathrm{C}_{6} \mathrm{~F}_{5}\right) \cdot{ }^{19} \mathrm{~F} /{ }^{19} \mathrm{~F}$ GCOSY (tetrahydrofuran- $\left.\mathrm{d}_{8}, 564 / 564 \mathrm{MHz}\right): \delta\left({ }^{19} \mathrm{~F}\right) / \delta$ $\left({ }^{19} \mathrm{~F}\right)=-132.6 /-135.8,-159.3(2-\mathrm{F} / 5-\mathrm{F}, 3-\mathrm{F}),-135.8 /-132.6,-151.7$ (5-F / 2-F, 4-F), -151.7 / 135.8, -159.3 (4-F / 5-F, 3-F), -159.3 / -132.6, -151.7 (3-F / 2-F, 4-F), -133.5 / -165.5 (o-F / m-F), 134.2 / -165.9 (o-F' / m-F'), -160.2 / -165.5 (p-F / m-F), -161.1 / -165.9 (p-F' / m-F'), -165.5 / 133.5, -160.2 (m-F/ o-F, p-F), -165.9 / -134.2, -161.1 (m-F' / o-F', p-F').

Reaction of complex 1 with phenyllithium, synthesis of 8. mp $114^{\circ} \mathrm{C}$. Anal. calcd. for $\mathrm{C}_{27} \mathrm{H}_{28} \mathrm{SiZr}$

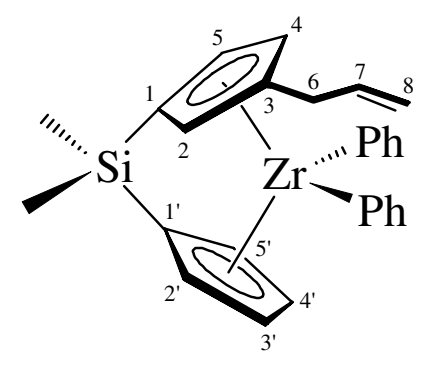

(471.8): C 68.73, H 5.98; found: C 67.42, H 5.67\%. IR (KBr): $\widetilde{v}=1623$ (w), $1413(w), 1308(w), 1246(m), 1162(m), 1085(m), 1029(s), 987(m)$, 931 (s), 854 (m), 805 (s), 721 (s), 700 (s), 665 (s), 450 (s) $\mathrm{cm}^{-1} .{ }^{1} \mathrm{H}-\mathrm{NMR}$ (d $\mathrm{d}_{8}$-toluene, $600 \mathrm{MHz}, 300 \mathrm{~K}$ ): $\delta=7.44,7.15,7.06$ (each m, 5H, o-, m-, p$\mathrm{H}$ of $\left.{ }^{\mathrm{b}} \mathrm{Ph}\right), 7.20,7.12,6.99$ (each m, 5H, o-, m-, p-H of $\left.{ }^{\mathrm{a}} \mathrm{Ph}\right), 6.48$ (m, 1H, 3H'), 6.37 (m, 1H, 4-H'), 6.34 (m, 1H, 4-H), 5.51 (m, 1H, 5-H'), 5.56 (m, 1H, 5-H), $5.54\left(\mathrm{~m}, 1 \mathrm{H}, 2-\mathrm{H}^{\prime}\right)$, 
$5.50(\mathrm{~m}, 1 \mathrm{H}, 7-\mathrm{H}), 5.32(\mathrm{~m}, 1 \mathrm{H}, 2-\mathrm{H}), 4.72\left(\mathrm{dm},{ }^{3} \mathrm{~J}_{\mathrm{HH}}=10.1 \mathrm{~Hz}, 1 \mathrm{H}, 8-\mathrm{H}\right), 4.64\left(\mathrm{dm},{ }^{3} \mathrm{~J}_{\mathrm{HH}}=17.0 \mathrm{~Hz}\right.$, 1H, 8-H'), 2.56/2.44 (each $\mathrm{ABX},{ }^{2} \mathrm{~J}_{\mathrm{HH}}=16.2 \mathrm{~Hz}$, each 1H, 6-H, 6-H'), $0.22\left(\mathrm{~s}, 6 \mathrm{H}, \mathrm{Si}\left(\mathrm{CH}_{3}\right)_{2}\right) .{ }^{13} \mathrm{C}\left\{{ }^{1} \mathrm{H}\right\}$ NMR ( $\mathrm{d}_{8}$-toluene, $150 \mathrm{MHz}$ ): $\delta=186.0,134.6,127.2,125.8$ (ipso-, o-, m-, p-C of ${ }^{\mathrm{a}} \mathrm{Ph}$ ), 181.7, 136.9, 126.5, 126.0 (ipso-, o-, m-, p-C of ' $\mathrm{Ph}$ ), 137.5 (C7), 135.7 (C3), 123.6 (C4'), 123.1 (C4), 121.4 (C3'), 115.2 (C8), 114.8 (C2), 114.1 (C2'), 113.7 (C5), 112.7 (C5'), 100.6 (C1), 100.2 (C1'), 34.1 (C6), -5.8 $\left(\mathrm{Si}\left(\mathrm{CH}_{3}\right)_{2}\right)$. 1D-TOCSY $\left(\mathrm{d}_{8}\right.$-toluene, $\left.600 / 600 \mathrm{MHz}\right): \delta\left({ }^{1} \mathrm{H}\right)_{\text {irr. }} / \delta\left({ }^{1} \mathrm{H}\right)_{\text {resp. }}=7.44 / 7.15,7.06\left(\mathrm{o}-\mathrm{H}\right.$ of ${ }^{\mathrm{b}} \mathrm{Ph} /$ m-, p-H of $\left.{ }^{b} \mathrm{Ph}\right), 7.29$ / 7.12, 6.99 (o-H of ${ }^{\mathrm{a}} \mathrm{Ph} / \mathrm{m}-, \mathrm{p}-\mathrm{H}$ of $\left.{ }^{\mathrm{a}} \mathrm{Ph}\right), 6.48$ / 6.37, 5.54, 5.51 (3-H' / 4-H', 2H', 5-H'), 5.32 / 6.34, 5.56 (2-H / 4-H, 5-H), 2.56, 2.44 / 5.50, 4.72, 4.64 (6-H, 6-H' / 7-H, 8-H, 8-H'). NOE-Diff $\left(\mathrm{d}_{8}\right.$-toluene, $\left.600 / 600 \mathrm{MHz}\right): \delta\left({ }^{1} \mathrm{H}\right)_{\text {irr. }} / \delta\left({ }^{1} \mathrm{H}\right)_{\text {resp. }}=7.44 / 7.20,7.15,6.37,6.34,5.56,5.51(\mathrm{o}-$ $\mathrm{H}$ of ${ }^{b} \mathrm{Ph} / \mathrm{o}-\mathrm{H}$ of ${ }^{\mathrm{a}} \mathrm{Ph}, \mathrm{m}-\mathrm{H}$ of $\left.{ }^{b} \mathrm{Ph}, 4-\mathrm{H}^{\prime}, 4-\mathrm{H}, 5-\mathrm{H}, 5-\mathrm{H}^{\prime}\right), 7.20$ / 7.44, 7.12, 6.48, 5.54, 5.32, 2.56, 2.44 (o-H of ${ }^{a} \mathrm{Ph} / \mathrm{o}-\mathrm{H}$ of ${ }^{b} \mathrm{Ph}, \mathrm{m}-\mathrm{H}$ of ${ }^{\mathrm{a}} \mathrm{Ph}, 3-\mathrm{H}^{\prime}$, 2-H', 2-H, 6-H, 6-H'), 6.48 / 7.20, 6.37, 5.54 (3-H' / o-H of ${ }^{\mathrm{a}} \mathrm{Ph}, 4-\mathrm{H}^{\prime}$, 2-H'), 6.37 / 7.44, 6.48, 5.51 (4-H' / o-H of ${ }^{\mathrm{b}} \mathrm{Ph}, 3-\mathrm{H}^{\prime}$, 5-H'), 6.34 / 7.44, 5.56, 2.56 (4-H / o$\mathrm{H}$ of $\left.{ }^{\mathrm{b}} \mathrm{Ph}, 5-\mathrm{H}, 6-\mathrm{H}\right), 5.56 / 7.44,7.20,6.48,6.34,\left(5-\mathrm{H} / \mathrm{o}-\mathrm{H}\right.$ of ${ }^{\mathrm{b}} \mathrm{Ph}, \mathrm{o}-\mathrm{H}$ of $\left.{ }^{\mathrm{a}} \mathrm{Ph}, 4-\mathrm{H}^{\prime}, 4-\mathrm{H}\right), 5.32$ / 7.20, 5.54, 2.44 (2-H / o-H of ${ }^{\mathrm{a}} \mathrm{Ph}, 2-\mathrm{H}^{\prime}$, 6-H'), 2.56 / 7.20, 6.34, 5.50, 5.32, 4.64, 2.44 (6-H / o-H of ${ }^{\mathrm{a}} \mathrm{Ph}, 4-$ H, 7-H, 2-H, 8-H', 6-H'), 2.44 / 7.20, 5.50, 5.32, 4.64, 2.56 (6-H' / o-H of 'Ph, 7-H, 2-H, 8-H', 6-H). GCOSY (d $\mathrm{d}_{8}$-toluene, $\left.600 / 600 \mathrm{MHz}, 300 \mathrm{~K}\right): \delta\left({ }^{1} \mathrm{H}\right) / \delta\left({ }^{1} \mathrm{H}\right)=6.48$ / 6.37, $5.54\left(3-\mathrm{H}^{\prime} / 4-\mathrm{H}^{\prime}, 2-\mathrm{H}^{\prime}\right)$, 6.37 / 6.48, 5.54 (4-H' / 3-H’, 2-H’), 6.34 / 5.56, 5.32 (4-H / 5-H, 2-H), 5.51 / 6.37 (5-H' / 4-H'), 5.56 / 6.34, 5.32 (5-H / 4-H, 2-H), 5.54 / 6.48 (2-H' / 3-H'), 5.32 / 5.56 (2-H / 5-H), 4.72 / 5.50 (8-H / 7-H), $2.56 / 5.50,2.44\left(6-\mathrm{H} / 7-\mathrm{H}, 6-\mathrm{H}^{\prime}\right)$. GHSQC (d -toluene, $\left.150 / 600 \mathrm{MHz}\right): \delta\left({ }^{13} \mathrm{C}\right) / \delta\left({ }^{1} \mathrm{H}\right)=134.6 / 7.20$ (o-C of ${ }^{a} \mathrm{Ph} / \mathrm{o}-\mathrm{H}$ of $\left.{ }^{\mathrm{a}} \mathrm{Ph}\right), 127.2$ / 7.12 (m-C of ${ }^{\mathrm{a}} \mathrm{Ph} / \mathrm{m}-\mathrm{H}$ of $\left.{ }^{\mathrm{a}} \mathrm{Ph}\right), 125.8 / 6.99$ (p-C of ${ }^{\mathrm{a}} \mathrm{Ph} / \mathrm{p}-\mathrm{H}$ of ${ }^{\mathrm{a}} \mathrm{Ph}$ ), 136.9 / 7.44 (o-C of ${ }^{b} \mathrm{Ph} / \mathrm{o}-\mathrm{H}$ of $\left.{ }^{b} \mathrm{Ph}\right), 126.5 / 7.15$ (m-C of ${ }^{b} \mathrm{Ph} / \mathrm{m}-\mathrm{H}$ of $\left.{ }^{b} \mathrm{Ph}\right), 126.0 / 7.06\left(\mathrm{p}-\mathrm{C}\right.$ of ${ }^{b} \mathrm{Ph} /$ p-H of $\left.{ }^{b} \mathrm{Ph}\right), 137.5$ / 5.50 (C7 / 7-H), 123.6 / 6.37 (C4' / 4-H'), 123.1 / 6.34 (C4 / 4-H), 121.4 / 6.48 (C3' / 3-H'), 115.2 / 4.72, 4.64 (C8 / 8-H, 8-H'), 114.8 / 5.32 (C2 / 2-H), 114.1 / 5.54 (C2' / 2-H'), 113.7 / 5.56 (C5 / 5-H), 112.7 / 5.51 (C5' / 5-H'), 34.1 / 2.56, 2.44 (C6 / 6-H, 6-H'), -5.8 / 0.22 $\left(\mathrm{Si}\left(\mathrm{CH}_{3}\right)_{2} / \mathrm{Si}\left(\mathrm{CH}_{3}\right)_{2}\right) . \mathrm{GHMBC}\left(\right.$ toluene-d $\left.\mathrm{d}_{8}, 150 / 600 \mathrm{MHz}\right): \delta\left({ }^{13} \mathrm{C}\right) / \delta\left({ }^{1} \mathrm{H}\right)=186.0 / 7.20,7.12$ (ipso- 
$\mathrm{C}$ of ${ }^{\mathrm{a}} \mathrm{Ph} / \mathrm{o}-, \mathrm{m}-\mathrm{H}$ of ${ }^{\mathrm{a}} \mathrm{Ph}$ ), 181.7 / 7.44, 7.15 (ipso-C of ${ }^{\mathrm{b}} \mathrm{Ph} / \mathrm{o}-, \mathrm{m}-\mathrm{H}$ of ${ }^{\mathrm{b}} \mathrm{Ph}$ ), 137.5 / 2.56, 2.44 (C7 /

6-H, 6-H'), 136.9 / 7.06 (o-C of ${ }^{b} \mathrm{Ph} / \mathrm{p}-\mathrm{H}$ of ${ }^{\mathrm{b}} \mathrm{Ph}$ ), 135.7 / 6.34, 5.56, 5.32, 2.56, 2.44 (C3 / 4-H, 5-H, 2-H, 6-H, 6-H'), 134.6 / 6.99 (o-C of ${ }^{\mathrm{a}} \mathrm{Ph} / \mathrm{p}-\mathrm{H}$ of ${ }^{\mathrm{a}} \mathrm{Ph}$ ), 126.0 / 7.44 (p-C of ${ }^{\mathrm{b}} \mathrm{Ph} / \mathrm{o}-\mathrm{H}$ of ${ }^{\mathrm{b}} \mathrm{Ph}$ ), 125.8 / 7.20 (p-C of ${ }^{\mathrm{a}} \mathrm{Ph} / \mathrm{o}-\mathrm{H}$ of $\left.{ }^{\mathrm{a}} \mathrm{Ph}\right), 123.6$ / 5.54 (C4' / 2-H'), 123.1 / 5.56, 5.32, 2.56, 2.44 (C4 / 5-H, 2-H, 6-H, 6-H'), 121.4 / 5.51 ( C3' / 5-H'), 114.8 / 6.34, 5.56, 2.56, 2.44 (C2 / 4-H, 5-H, 6-H, 6-H'), 114.1 / 5.51 (C2' / 5-H'), 112.7 / 5.54 (C5' / 2-H'), 113.7 / 5.32 (C5 / 2-H), 100.6 / 6.34, 5.56, 5.32, 0.22 (C1 / 4-H, 5-H, 2-H, Si( $\left.\left(\mathrm{CH}_{3}\right)_{2}\right), 100.2$ / 5.54, 5.51, $0.22\left(\mathrm{Cl}^{\prime}\right.$ / 2-H', 5-H', $\left.\mathrm{Si}\left(\mathrm{CH}_{3}\right)_{2}\right), 34.1$ / 5.50, 4.72, 4.64 (C6 / 7-H, 8-H, 8-H'), -5.8 / $0.22\left(\mathrm{Si}_{(}\left(\mathrm{CH}_{3}\right)_{2} / \mathrm{Si}\left(\mathrm{CH}_{3}\right)_{2}\right)$.

\section{Thermolysis of complex 8, formation of the products 10 and 11 . NMR experiment: ${ }^{1} \mathrm{H}-\mathrm{NMR}$}

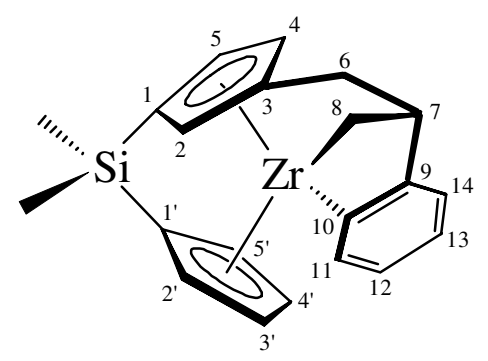
(cyclohexane- $\left.\mathrm{d}_{12}, 600 \mathrm{MHz}\right): \delta=7.12\left(\mathrm{~m}, 1 \mathrm{H}, 3-\mathrm{H}^{\prime}\right), 6.89(\mathrm{~m}, 1 \mathrm{H}, 4-$ H'), $6.83(\mathrm{~m}, 1 \mathrm{H}, 12-\mathrm{H}), 6.82(\mathrm{~m}, 1 \mathrm{H}, 11-\mathrm{H}), 6.74(\mathrm{~m}, 1 \mathrm{H}, 14-\mathrm{H}), 6.72$ (m, 1H, 13-H), $6.20(\mathrm{~m}, 1 \mathrm{H}, 4-\mathrm{H}), 5.69\left(\mathrm{~m}, 1 \mathrm{H}, 5-\mathrm{H}^{\prime}\right), 5.64(\mathrm{~m}, 1 \mathrm{H}, 2-$ H'), $5.60(\mathrm{~m}, 1 \mathrm{H}, 5-\mathrm{H}), 5.19(\mathrm{~m}, 1 \mathrm{H}, 2-\mathrm{H}), 3.68(\mathrm{~m}, 1 \mathrm{H}, 7-\mathrm{H}), 2.97 / 2.83$ (each m, each 1H, 6-H, 6-H'), 1.19/0.60 (each m, each 1H, 8-H, 8-H'), 0.56/0.40 (each s, each 3H, $\left.\mathrm{Si}\left(\mathrm{CH}_{3}\right)_{2}\right) .{ }^{13} \mathrm{C}\left\{{ }^{1} \mathrm{H}\right\}-\mathrm{NMR}$ (cyclohexane-d $\left.\mathrm{d}_{12}, 150 \mathrm{MHz}\right): \delta=181.2(\mathrm{C} 10)$, 156.0 (C9), 134.9 (C11), 130.4 (C3), 127.2 (C12), 126.8 (C14), 124.6 (C13), 124.5 (C4), 120.2 (C3'), 117.6 (C4'), 116.5 (C2), 113.7 (C2'), 112.2 (C5'), 110.2 (C5), 102.1 (C1'), 98.0 (C1), 57.8 (C8), 45.5

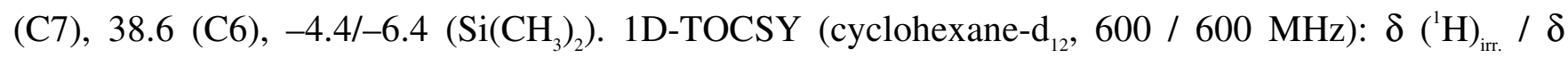
$\left({ }^{\prime} \mathrm{H}\right)_{\text {resp. }}=7.12$ / 6.89, 5.69, $5.64\left(3-\mathrm{H}^{\prime} / 4-\mathrm{H}^{\prime}, 5-\mathrm{H}^{\prime}, 2-\mathrm{H}^{\prime}\right), 5.19$ / 6.20, $5.60(2-\mathrm{H} / 4-\mathrm{H}, 5-\mathrm{H}), 6.83,6.82$ / 6.74, 6.72 (12-H, 11-H / 14-H, 13-H), 3.68 / 2.97, 2.83, 1.19, 0.60 (7-H / 6-H, 6-H', 8-H, 8-H'). NOE-Diff (cyclohexane-d $\left.{ }_{12}, 600 / 600 \mathrm{MHz}\right): \delta\left({ }^{1} \mathrm{H}\right)_{\text {irr. }} / \delta\left({ }^{1} \mathrm{H}\right)_{\text {resp. }}=7.12$ / 6.89, 5.64, $1.19\left(3-\mathrm{H}^{\prime} / 4-\mathrm{H}^{\prime}\right.$, 2-H’, 8-H), 6.89 / 7.12, 5.69 (4-H’ / 3-H’, 5-H'), 6.72 / 6.83, 3.68 (13-H / 12-H, 7-H), 6.20 / 5.60, 2.83 (4-H / 5-H, 6-H'), 5.69 / 6.89, 0.56 (5-H' / 4-H', $\left.\mathrm{Si}\left(\mathrm{CH}_{3}\right)_{2}\right), 5.64$ / 7.12, 0.40 (2-H' / 3-H', $\left.\mathrm{Si}\left(\mathrm{CH}_{3}\right)_{2}\right)$, 5.60 / 6.20, 0.56 (5-H / 4-H, Si( $\left.\left(\mathrm{CH}_{3}\right)_{2}\right), 5.19$ / 2.97, 0.60, 0.40 (2-H / 6-H, 8-H', Si( $\left.\left(\mathrm{CH}_{3}\right)_{2}\right), 3.68$ / 6.74, 2.97, 2.83, 1.19, 0.60 (7-H / 14-H, 6-H, 6-H', 8-H, 8-H’), 2.97 / 5.19, 3.68, 2.83, 0.60 (6-H / 2-H, 7-H, 
6-H’, 8-H’), 2.83 / 6.74, 6.20, 3.68, 2.97 (6-H' / 14-H, 4-H, 7-H, 6-H), 1.19 / 5.64, 3.68 ,0.60 (8-H / 2H', 7-H, Si( $\left.\left(\mathrm{CH}_{3}\right)_{2}\right), 0.60$ / 5.19, 3.68, 2.97, 1.19 (8-H' / 2-H, 7-H, 6-H, 8-H'), 0.56 / 5.69, 5.60 $\left(\mathrm{Si}\left(\mathrm{CH}_{3}\right)_{2} /\right.$ 5-H', 5-H), 0.40 / 5.64, $5.19\left(\mathrm{Si}_{\left(\mathrm{CH}_{3}\right)_{2} / \text { 2-H', 2-H). GCOSY (cyclohexane-d }}, 600 / 600\right.$ MHz): $\delta\left({ }^{1} \mathrm{H}\right) / \delta\left({ }^{1} \mathrm{H}\right)=7.12$ / 6.89, 5.69, 5.64 (3-H' / 4-H', 5-H', 2-H'), 6.89 / 7.12, 5.69, 5.64 (4-H' / 3-H', 5-H', 2-H'), 6.83, 6.82 / 6.74, 6.72 (12-H, 11-H / 14-H, 13-H), 6.74 / 6.83, 6.82 (14-H / 12-H, 11-H), 6.20 / 5.60 (4-H / 5-H), 5.69 / 6.89 (5-H’ / 4-H’), 5.64 / 7.12 (2-H’ / 3-H'), 5.60 / 6.20, 5.19 (5H / 4-H, 2-H), 5.19 / 5.60 (2-H / 5-H), 3.68 / 2.97, 0.60 (7-H / 6-H, 8-H'), 2.97 / 3.68, 2.83 (6-H / 7-H, 6-H’), 2.83 / 2.97, 1.19 (6-H’ / 6-H, 8-H), 1.19 / 3.68, 0.60 (8-H / 7-H, 8-H’), 0.60 / 3.68, 1.19 (6-H’ / 7-H, 6-H). GHSQC (cyclohexane-d $\left.{ }_{12}, 150 / 600 \mathrm{MHz}\right): \delta\left({ }^{13} \mathrm{C}\right) / \delta\left({ }^{1} \mathrm{H}\right)=134.9 / 7.20(\mathrm{C} 11 / 11-\mathrm{H})$, 127.2 / 6.83 (C12 / 12-H), 126.8 / 6.74 (C14 / 14-H), 124.6 /6.72 (C13 / 13-H), 124.5 / 6.20 (C4 / 4 H), 117.6 / 6.89 (C4' / 4-H'), 120.2 / 7.12 (C3' / 3-H'), 116.5 / 5.19 (C2 / 2-H), 113.7 / 5.64 (C2' / 2H'), 112.2 / 5.69 (C5' / 5-H'), 110.2 / 5.60 (C5 / 5-H), 57.8 / 1.19, 0.60 (C8 / 8-H, 8-H'), 45.5 / 3.68 (C7 / 7-H), 38.6 / 2.97, $2.83\left(\mathrm{C} 6 / 6-\mathrm{H}, 6-\mathrm{H}^{\prime}\right),-4.4$ / $0.56\left(\mathrm{Si}\left(\mathrm{CH}_{3}\right)_{2} / \mathrm{Si}\left(\mathrm{CH}_{3}\right)_{2}\right),-6.4 / 0.40\left(\mathrm{Si}\left(\mathrm{CH}_{3}\right)_{2} /\right.$ $\left.\operatorname{Si}\left(\mathrm{CH}_{3}\right)_{2}\right)$. GHMBC $\left(\right.$ cyclohexane-d $\left.{ }_{12}, 150 / 600 \mathrm{MHz}\right): \delta\left({ }^{13} \mathrm{C}\right) / \delta\left({ }^{1} \mathrm{H}\right)=181.2 / 6.83,6.74,6.72(\mathrm{C} 10 /$ 12-H, 14-H, 13-H), 156.0 / 6.83, 6.82, 2.97, 2.83, 0.60 (C9 / 12-H, 11-H, 6-H, 6-H', 8-H'), 134.9 / 6.83, 2.97 (C11 / 12-H, 6-H), 130.4 / 5.19, 2.97, 2.83 (C3 / 2-H, 6-H, 6-H'), 127.2 / 6.82 (C12 / 11-H), 126.8 / 6.72 (C14 / 13-H), 124.6 / 6.83, 6.74, 2.97 (C13 / 12-H, 14-H, 6-H), 124.5 / 5.19, 2.97, 2.83 (C4 / 2-H, 6-H, 6-H'), 120.2 / 5.69 ( C3' / 5-H'), 116.5 / 2.97, 2.83 (C2 / 6-H, 6-H'), 110.2 / 5.19 (C5 / 2-H), 102.1 / 5.64, 0.56, $0.40\left(\mathrm{Cl}^{\prime} /\right.$ 2-H', $\left.\mathrm{Si}\left(\mathrm{CH}_{3}\right)_{2}, \mathrm{Si}\left(\mathrm{CH}_{3}\right)_{2}\right), 98.0$ / 5.19, 0.56, $0.40(\mathrm{C} 1 / 2-\mathrm{H}$, $\left.\mathrm{Si}\left(\mathrm{CH}_{3}\right)_{2}, \mathrm{Si}\left(\mathrm{CH}_{3}\right)_{2}\right), 57.8$ / 2.83 (C8 / 6-H'), 45.5 / 6.74, 2.97, 2.83, 0.60 (C7 /14-H, 6-H, 6-H', 8-H'), 38.6 / 1.19, 0.60 (C6 / 8-H, 8-H'), -4.4 / $0.40\left(\mathrm{Si}\left(\mathrm{CH}_{3}\right)_{2} / \mathrm{Si}\left(\mathrm{CH}_{3}\right)_{2}\right),-6.4$ / $0.56\left(\mathrm{Si}\left(\mathrm{CH}_{3}\right)_{2} / \mathrm{Si}\left(\mathrm{CH}_{3}\right)_{2}\right)$.

Formation of 11 on a preparative scale. $\mathrm{mp} 132^{\circ} \mathrm{C}$. Anal. calcd. for $\mathrm{C}_{21} \mathrm{H}_{24} \mathrm{Cl}_{2} \mathrm{SiZr}$ (466.6): C

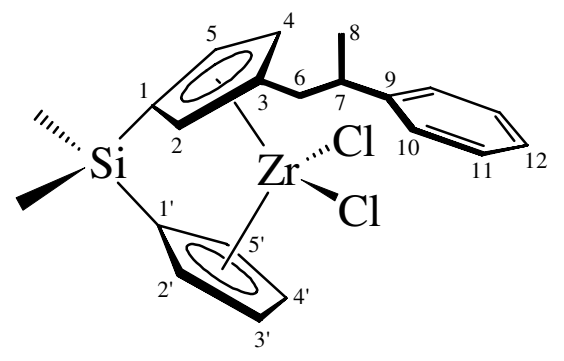
54.05, H 5.18; found: C 53.74, H 5.05\%. IR (KBr): $\widetilde{v}=3032(\mathrm{w})$, $2956(\mathrm{~m}), 2831(\mathrm{~m}), 1645(\mathrm{w}), 1463(\mathrm{~s}), 1364(\mathrm{~m}), 1265(\mathrm{~s}), 1480$ 
(m), $1057(\mathrm{~m}), 833(\mathrm{~s}), 824(\mathrm{~m}), 693(\mathrm{w}), 456(\mathrm{w}) \mathrm{cm}^{-1} .{ }^{1} \mathrm{H}-\mathrm{NMR}$ (benzene-d $\left.{ }_{6}, 600 \mathrm{MHz}\right): \delta=7.08(\mathrm{~m}$, 2H, 11-H), $7.02(\mathrm{~m}, 1 \mathrm{H}, 12-\mathrm{H}), 6.92(\mathrm{~m}, 2 \mathrm{H}, 10-\mathrm{H}), 6.85\left(\mathrm{~m}, 1 \mathrm{H}, 3-\mathrm{H}^{\prime}\right), 6.75\left(\mathrm{~m}, 1 \mathrm{H}, 4-\mathrm{H}^{\prime}\right), 6.26$ (m, 1H, 4-H), 5.49 (m, 1H, 5-H'), 5.40 (m, 1H, 2-H'), 5.39 (m, 1H, 5-H), 5.18 (m, 1H, 2-H), 3.06/3.00 (each m, each 1H, 6-H, 6-H'), $2.76(\mathrm{~m}, 1 \mathrm{H}, 7-\mathrm{H}), 1.17\left(\mathrm{~d},{ }^{3} \mathrm{~J}_{\mathrm{HH}}=7.0 \mathrm{~Hz}, 3 \mathrm{H}, 8-\mathrm{H}\right), 0.10 / 0.06$ (each s, each 3H, Si $\left.\left(\mathrm{CH}_{3}\right)_{2}\right) .{ }^{13} \mathrm{C}\left\{{ }^{1} \mathrm{H}\right\}-\mathrm{NMR}$ (benzene-d, $\left.150 \mathrm{MHz}\right): \delta=146.2(\mathrm{C} 9), 140.7(\mathrm{C} 3), 128.9(\mathrm{C} 4)$, 128.5 (C11), 127.7 (C4’), 127.4 (C3’), 127.3 (C10), 126.2 (C12), 115.7 (C2'), 114.8 (C5), 114.2 (C2), 113.7 (C5'), 107.7 (C1), 107.1 (C1'), 41.8 (C7), 39.4 (C6), 22.1 (C8), -5.8/-6.1 (Si(CH$\left.)_{2}\right)$. 1DTOCSY (benzene-d $\left.{ }_{6}, 600 / 600 \mathrm{MHz}\right): \delta\left({ }^{1} \mathrm{H}\right)_{\text {irr. }} / \delta\left({ }^{1} \mathrm{H}\right)_{\text {resp. }}=6.92 / 7.08,7.02(10-\mathrm{H} / 11-\mathrm{H}, 12-\mathrm{H}), 5.49$ / 6.85, 6.75, 5.40 (5-H' / 3-H', 4-H', 2-H'), 5.18 / 6.26, 5.39 (2-H / 4-H, 5-H), 2.76 / 3.06, 3.00, 1.17 (7-H / 6-H, 6-H', 8-H). NOE-Diff (benzene-d $\left.{ }_{6}, 600 / 600 \mathrm{MHz}\right): \delta\left({ }^{1} \mathrm{H}\right)_{\text {irr. }} / \delta\left({ }^{1} \mathrm{H}\right)_{\text {resp. }}=6.75 / 6.85,5.49$ (4-H’ / 3-H', 5-H’), 5.49 / 6.75, 1.17, 0.06 (5-H’ / 4-H’, 8-H, Si( $\left.\left(\mathrm{CH}_{3}\right)_{2}\right)$, 5.40, 5.39 / 6.85, 6.26, 1.17, 0.10, 0.06 (2-H', 5-H / 3-H', 4-H, 8-H, Si( $\left.\left(\mathrm{CH}_{3}\right)_{2}\right), 5.18$ / 5.40, 3.06, 1.17, 0.10 (2-H / 2-H', 6-H, 8-H, $\left.\mathrm{Si}\left(\mathrm{CH}_{3}\right)_{2}\right), 3.06$ / 6.92, 5.18, 3.00, 2.76, 1.17 (6-H / 10-H, 2-H, 6-H', 7-H, 8-H), 3.00 / 6.92, 6.26, 3.06, 2.76, 1.17 (6-H' / 10-H, 4-H, 6-H, 7-H, 8-H), 1.17 / 6.92, 3.06, 2.76 (8-H / 10-H, 6-H, 7-H), 0.10 / 5.40, $5.18\left(\mathrm{Si}\left(\mathrm{CH}_{3}\right)_{2} / 2-\mathrm{H}^{\prime}, 2-\mathrm{H}\right), 0.06$ / 5.49, $5.39\left(\mathrm{Si}_{\left(\mathrm{CH}_{3}\right)_{2} / \text { 5-H', 5-H). GCOSY (benzene-d }}, 600\right.$ / $600 \mathrm{MHz}): \delta\left({ }^{1} \mathrm{H}\right) / \delta\left({ }^{1} \mathrm{H}\right)=7.08 / 7.02,6.92(11-\mathrm{H} / 12-\mathrm{H}, 10-\mathrm{H}), 7.02 / 7.08$ (12-H / 11-H), $6.92 /$ 7.08 (10-H / 11-H), 6.85 / 6.75, 5.49, 5.40 (3-H' / 4-H’, 5-H’, 2-H'), 6.75 / 6.85, 5.49, 5.40 (4-H’ / 3H', 5-H', 2-H'), 6.26 / 5.39, 5.18 (4-H / 5-H, 2-H), 5.49 / 6.85, 6.75, 5.40 (5-H' / 3-H', 4-H', 2-H'), 5.40 / 6.85, 6.75, 5.49 (2-H' / 3-H', 4-H', 5-H'), 5.39 / 6.26, 5.18 (5-H / 4-H, 2-H), 5.18 / 6.26, 5.39 (2-H / 4-H, 5-H), 3.06 / 3.00, 2.76 (6-H / 6-H', 7-H), 3.00 / 3.06, 2.76 (6-H' / 6-H, 7-H), 2.76 / 3.06 , 3.00, 1.17 (7-H / 6-H, 6-H', 8-H), 1.17 / 2.76 (8-H / 7-H). GHSQC (benzene-d, 150 / 600 MHz): $\delta$ $\left({ }^{13} \mathrm{C}\right) / \delta\left({ }^{1} \mathrm{H}\right)=128.9 / 6.26(\mathrm{C} 4 / 4-\mathrm{H}), 128.5 / 7.08(\mathrm{C} 11 / 11-\mathrm{H}), 127.7 / 6.75(\mathrm{C} 4$ ' / 4-H'), $127.4 /$ 6.85 (C3' / 3-H'), 127.3 / 6.92 (C10 / 10-H), 126.2 / 7.02 (C12 / 12-H), 115.7 / 5.40 (C2' / 2-H'), 114.8 / 5.39 (C5 / 5-H), 114.2 / 5.18 (C2 / 2-H), 113.7 / 5.49 (C5' / 5-H'), 22.1 / 1.17 (C8 / 8-H), 41.8 / $2.76\left(\mathrm{C} 7\right.$ / 7-H), 39.4 / 3.06, 3.00 (C6 / 6-H, 6-H'), -5.8 / $0.10\left(\mathrm{Si}\left(\mathrm{CH}_{3}\right)_{2} / \mathrm{Si}\left(\mathrm{CH}_{3}\right)_{2}\right),-6.1 / 0.06$ 
$\left(\mathrm{Si}\left(\mathrm{CH}_{3}\right)_{2} / \mathrm{Si}\left(\mathrm{CH}_{3}\right)_{2}\right) . \mathrm{GHMBC}\left(\right.$ benzene-d $\left._{6}, 150 / 600 \mathrm{MHz}\right): \delta\left({ }^{13} \mathrm{C}\right) / \delta\left({ }^{1} \mathrm{H}\right)=146.2 / 7.08,3.06,3.00$, 2.76, 1.17 (C9 / 11-H, 6-H, 6-H', 7-H, 8-H), 140.7 / 3.06, 3.00 (C3 / 6-H, 6-H'), 128.9 / 3.06, 3.00 (C4 / 6-H, 6-H'), 128.5 / 7.08 (C11 / 11-H), 127.3 / 7.02, 6.92, 2.76 (C10 / 12-H, 10-H, 7-H), 126.2 / 6.92 (C12 / 10-H), 114.8 / 5.18 (C5 / 2-H), 114.2 / 3.06, 3.00 (C2 / 6-H, 6-H'), 107.7 / 5.18, 0.10, 0.06 (C1 / 2-H, $\left.\mathrm{Si}\left(\mathrm{CH}_{3}\right)_{2}, \mathrm{Si}\left(\mathrm{CH}_{3}\right)_{2}\right), 107.1$ / 5.40, 0.10, $0.06\left(\mathrm{Cl}^{\prime}\right.$ / 2-H', $\left.\mathrm{Si}\left(\mathrm{CH}_{3}\right)_{2}, \mathrm{Si}\left(\mathrm{CH}_{3}\right)_{2}\right), 41.8$ / 6.92, 3.06, 3.00, 1.17 (C7 / 10-H, 6-H, 6-H', 8-H), 39.4 / 2.76, 1.17 (C6 / 7-H, 8-H), 22.1 / 3.06, 3.00, 2.76 (C8 / 6-H, 6-H', 7-H), -5.8 / $0.06\left(\mathrm{Si}\left(\mathrm{CH}_{3}\right)_{2} / \mathrm{Si}\left(\mathrm{CH}_{3}\right)_{2}\right),-6.1 / 0.10\left(\mathrm{Si}\left(\mathrm{CH}_{3}\right)_{2} / \mathrm{Si}\left(\mathrm{CH}_{3}\right)_{2}\right)$.

Addition of $\mathrm{HB}\left(\mathbf{C}_{6} \mathbf{F}_{5}\right)_{2}$ to complex 8. generation of 12. ${ }^{1} \mathrm{H}-\mathrm{NMR}$ (toluene- $\left.\mathrm{d}_{8}, 600 \mathrm{MHz}\right): \delta=7.41$,

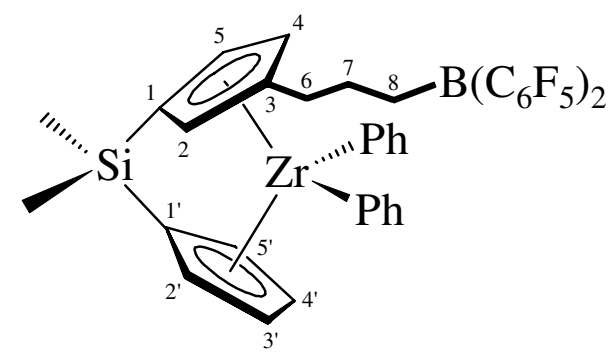
7.15, 7.06 (each m, 5H, o-, m-, p-H of ${ }^{\mathrm{b}} \mathrm{Ph}$ ), 7.20, 7.14, 7.02 (each m, 5H, o-, m-, p-H of $\left.{ }^{\mathrm{a}} \mathrm{Ph}\right), 6.46\left(\mathrm{~m}, 1 \mathrm{H}, 3-\mathrm{H}^{\prime}\right), 6.34$ (m, 1H, 4-H'), $6.31(\mathrm{~m}, 1 \mathrm{H}, 4-\mathrm{H}), 5.55(\mathrm{~m}, 1 \mathrm{H}, 5-\mathrm{H}), 5.54(\mathrm{~m}, 1 \mathrm{H}$, 2-H'), 5.48 (m, 1H, 5-H'), 5.29 (m, 1H, 2-H), 1.88/1.70 (each m, each 1H, 6-H, 6-H'), 1.62 (m, 2H, 8-H), $1.40(\mathrm{~m}, 2 \mathrm{H}, 7-\mathrm{H})$, $0.23\left(\mathrm{~s}, 6 \mathrm{H}, \mathrm{Si}\left(\mathrm{CH}_{3}\right)_{2}\right) .{ }^{13} \mathrm{C}\left\{{ }^{1} \mathrm{H}\right\}-\mathrm{NMR}$ (toluene-d ${ }_{8}, 150 \mathrm{MHz}$ ): $\delta=186.0,134.6,127.3,125.8$ (ipso-, o-, m-, p-C of ${ }^{a} \mathrm{Ph}$ ), 181.6, 136.0, 126.6, 126.2 (ipso-, o-, m-, p-C of ${ }^{b} \mathrm{Ph}$ ), 136.9 (C3), 123.9 (C4'), 122.6 (C4), 121.5 (C3'), 114.3 (C2'), 114.2 (C2), 113.8 (C5), 112.7 (C5'), 101.1 (C1), 100.3 (C1'), 32.3 (C6), $31.8(\mathrm{C} 8), 26.9(\mathrm{C} 7),-5.8\left(\mathrm{Si}\left(\mathrm{CH}_{3}\right)_{2}\right) ; 147.0\left({ }^{1} \mathrm{~J}_{\mathrm{CF}}=250.1 \mathrm{~Hz}, \mathrm{o}-\mathrm{C}\right.$ of $\left.\mathrm{C}_{6} \mathrm{~F}_{5}\right), 143.4\left({ }^{1} \mathrm{~J}_{\mathrm{CF}}=253.2 \mathrm{~Hz}\right.$, $\mathrm{p}-\mathrm{C}$ of $\left.\mathrm{C}_{6} \mathrm{~F}_{5}\right), 137.6\left({ }^{1} \mathrm{~J}_{\mathrm{CF}}=250.1 \mathrm{~Hz}, \mathrm{~m}-\mathrm{C}\right.$ of $\left.\mathrm{C}_{6} \mathrm{~F}_{5}\right) .{ }^{11} \mathrm{~B}\left\{{ }^{1} \mathrm{H}\right\}-\mathrm{NMR}\left(\right.$ toluene- $\left.\mathrm{d}_{8}, 64 \mathrm{MHz}\right): \delta=79.2 .{ }^{19} \mathrm{~F}-$ NMR (toluene- $\left.\mathrm{d}_{8}, 564 \mathrm{MHz}\right): \delta=-129.7\left(\mathrm{~m}, 4 \mathrm{~F}\right.$, o-F of $\left.\mathrm{C}_{6} \mathrm{~F}_{5}\right),-147.5\left(\mathrm{t},{ }^{3} \mathrm{~J}_{\mathrm{FF}}=21.7 \mathrm{~Hz}, 2 \mathrm{~F}, \mathrm{p}-\mathrm{F}\right.$ of $\left.\mathrm{C}_{6} \mathrm{~F}_{5}\right),-160.9\left(\mathrm{~m}, 4 \mathrm{~F}, \mathrm{~m}-\mathrm{F}\right.$ of $\left.\mathrm{C}_{6} \mathrm{~F}_{5}\right)$. 1D-TOCSY (toluene-d $\left.\mathrm{d}_{8}, 600 / 600 \mathrm{MHz}\right): \delta\left({ }^{1} \mathrm{H}\right)_{\text {irr. }} / \delta\left({ }^{1} \mathrm{H}\right)_{\text {resp. }}=7.41$ / 7.15, $7.06\left(\mathrm{o}-\mathrm{H}\right.$ of ${ }^{\mathrm{b}} \mathrm{Ph} / \mathrm{m}-, \mathrm{p}-\mathrm{H}$ of $\left.{ }^{\mathrm{b}} \mathrm{Ph}\right), 7.20 / 7.14,7.02\left(\mathrm{o}-\mathrm{H}\right.$ of ${ }^{\mathrm{a}} \mathrm{Ph} / \mathrm{m}-, \mathrm{p}-\mathrm{H}$ of $\left.{ }^{\mathrm{a}} \mathrm{Ph}\right), 5.48 / 6.46$, 6.34, 5.54 (5-H' / 3-H', 4-H’, 2-H’), 5.29 / 6.31, 5.55 (2-H / 4-H, 5-H), 1.88 / 1.70, 1.62, 1.40 (6-H / 6-H’, 8-H, 7-H). NOE-Diff (toluene-d $\left.{ }_{8}, 600 / 600 \mathrm{MHz}\right): \delta\left({ }^{1} \mathrm{H}\right)_{\text {irr. }} / \delta\left({ }^{1} \mathrm{H}\right)_{\text {resp. }}=7.41 / 7.20,7.15,6.34$, 6.31, 5.55, 5.48 (o-H of ${ }^{b} \mathrm{Ph} / \mathrm{o}-\mathrm{H}$ of ${ }^{\mathrm{a}} \mathrm{Ph}, \mathrm{m}-\mathrm{H}$ of $\left.{ }^{\mathrm{b}} \mathrm{Ph}, 4-\mathrm{H}^{\prime}, 4-\mathrm{H}, 5-\mathrm{H}, 5-\mathrm{H}^{\prime}\right), 7.20$ / 7.41, 7.14, 6.46, 
5.54, 5.29 (o-H of ${ }^{\mathrm{a}} \mathrm{Ph} / \mathrm{o}-\mathrm{H}$ of ${ }^{\mathrm{b}} \mathrm{Ph}, \mathrm{m}-\mathrm{H}$ of $\left.{ }^{\mathrm{a}} \mathrm{Ph}, 3-\mathrm{H}^{\prime}, 2-\mathrm{H}^{\prime}, 2-\mathrm{H}\right), 6.46$ / 7.20, 6.34, 5.54 (3-H' / o-H of $\left.{ }^{\mathrm{a}} \mathrm{Ph}, 4-\mathrm{H}^{\prime}, 2-\mathrm{H}^{\prime}\right), 6.34$ / 7.41, 6.46, 5.48 (4-H’ / o-H of ${ }^{\mathrm{b}} \mathrm{Ph}, 4-\mathrm{H}^{\prime}$, 5-H'), 6.31 / 7.41, 5.55, 1.88 (4-H / o$\mathrm{H}$ of $\left.{ }^{b} \mathrm{Ph}, 5-\mathrm{H}, 6-\mathrm{H}\right), 5.55,5.54 / 7.41,7.20,6.46,6.31,5.29,0.23$ (5-H, 2-H' / o-H of ${ }^{b} \mathrm{Ph}, \mathrm{o}-\mathrm{H}$ of ${ }^{\mathrm{a}} \mathrm{Ph}$, 3-H', 4-H, 2-H, Si $\left.\left(\mathrm{CH}_{3}\right)_{2}\right), 5.48$ / 7.41, 6.34, 0.23 (5-H' / o-H of $\left.{ }^{b} \mathrm{Ph}, 4-\mathrm{H}^{\prime}, \mathrm{Si}\left(\mathrm{CH}_{3}\right)_{2}\right), 5.29$ / 7.20, 5.54, 1.70 (2-H / o-H of $\left.{ }^{\mathrm{a}} \mathrm{Ph}, 2-\mathrm{H}^{\prime}, \mathrm{6}^{-\mathrm{H}^{\prime}}\right), 1.88$ / 6.31 (6-H / 4-H), 1.70 / 5.29 (6-H' / 2-H), 0.23 / 5.55, 5.54, 5.48, $5.29\left(\mathrm{Si}_{\left(\mathrm{CH}_{3}\right)_{2} / 5-\mathrm{H}, 2-\mathrm{H}^{\prime}, \text { 5-H', 2-H). GCOSY (toluene-d }}, 600 / 600 \mathrm{MHz}\right): \delta\left({ }^{1} \mathrm{H}\right) / \delta\left({ }^{1} \mathrm{H}\right)=$ $7.41 / 7.15$ (o-H of ${ }^{b} \mathrm{Ph} / \mathrm{m}-\mathrm{H}$ of $\left.{ }^{b} \mathrm{Ph}\right), 7.20 / 7.14$ (o-H of ${ }^{\mathrm{a}} \mathrm{Ph} / \mathrm{m}-\mathrm{H}$ of $\left.{ }^{\mathrm{a}} \mathrm{Ph}\right), 7.15 / 7.41,7.06$ (m-H of ${ }^{b} \mathrm{Ph} / \mathrm{o}-\mathrm{H}$ of ${ }^{b} \mathrm{Ph}, \mathrm{p}-\mathrm{H}$ of $\left.{ }^{b} \mathrm{Ph}\right), 7.14 / 7.20,7.02\left(\mathrm{~m}-\mathrm{H}\right.$ of ${ }^{\mathrm{a}} \mathrm{Ph} / \mathrm{o}-\mathrm{H}$ of ${ }^{\mathrm{a}} \mathrm{Ph}, \mathrm{p}-\mathrm{H}$ of $\left.{ }^{\mathrm{a}} \mathrm{Ph}\right), 6.46 / 6.34,5.54$ (3-H' / 4-H', 2-H'), 6.34 / 6.46, 5.54 (4-H' / 3-H', 2-H'), 6.31 / 5.55, 5.29 (4-H / 5-H, 2-H), 5.48 / 6.34, 5.54 (5-H' / 4-H', 2-H'), 5.55 / 6.31, 5.29 (5-H / 4-H, 2-H), 5.54 / 6.46, 5.48 (2-H’ / 3-H', 5-H'), 5.29 / 6.31, 5.55 (2-H / 4-H, 5-H), 1.88 / 1.70, 1.40 (6-H / 6-H’, 7-H), 1.70 / 1.88, 1.62, 1.40 (6-H' / 6H, 8-H, 7-H), 1.62 / 1.70, 1.40 (8-H / 6-H', 7-H), 1.40 / 1.88, 1.70, 1.62 (7-H / 6-H, 6-H', 8-H). GHSQC (toluene- $\left.\mathrm{d}_{8}, 150 / 600 \mathrm{MHz}\right): \delta\left({ }^{13} \mathrm{C}\right) / \delta\left({ }^{1} \mathrm{H}\right)=134.6 / 7.20\left(\mathrm{o}-\mathrm{C}\right.$ of ${ }^{\mathrm{a}} \mathrm{Ph} / \mathrm{o}-\mathrm{H}$ of $\left.{ }^{\mathrm{a}} \mathrm{Ph}\right), 127.3 /$ $7.14\left(\mathrm{~m}-\mathrm{C}\right.$ of ${ }^{\mathrm{a}} \mathrm{Ph} / \mathrm{m}-\mathrm{H}$ of $\left.{ }^{\mathrm{a}} \mathrm{Ph}\right), 125.8 / 7.02\left(\mathrm{p}-\mathrm{C}\right.$ of ${ }^{\mathrm{a}} \mathrm{Ph} / \mathrm{p}-\mathrm{H}$ of $\left.{ }^{\mathrm{a}} \mathrm{Ph}\right), 136.0 / 7.41$ (o-C of ${ }^{\mathrm{b}} \mathrm{Ph} / \mathrm{o}-\mathrm{H}$ of $\left.{ }^{b} \mathrm{Ph}\right), 126.6$ / $7.15\left(\mathrm{~m}-\mathrm{C}\right.$ of ${ }^{b} \mathrm{Ph} / \mathrm{m}-\mathrm{H}$ of $\left.{ }^{b} \mathrm{Ph}\right), 126.2$ / $7.06\left(\mathrm{p}-\mathrm{C}\right.$ of ${ }^{b} \mathrm{Ph} / \mathrm{p}-\mathrm{H}$ of $\left.{ }^{b} \mathrm{Ph}\right), 123.9$ / 6.34 (C4' / 4-H'), 122.6 / 6.31 (C4 / 4-H), 121.5 / 6.46 (C3' / 3-H'), 114.3 / 5.54 (C2' / 2-H'), 114.2 / 5.29 (C2 / 2 H), 113.8 / 5.55 (C5 / 5-H), 112.7 / 5.48 (C5' / 5-H'), 32.3 / 1.88, 1.70 (C6 / 6-H, 6-H'), 31.8 / 1.62 (C8 / 8-H), 26.9 / $1.40(\mathrm{C} 7 / 7-\mathrm{H}),-5.8 / 0.23\left(\mathrm{Si}\left(\mathrm{CH}_{3}\right)_{2} / \mathrm{Si}\left(\mathrm{CH}_{3}\right)_{2}\right) . \mathrm{GHMBC}$ (toluene-d ${ }_{8}, 150 / 600$ $\mathrm{MHz}): \delta\left({ }^{13} \mathrm{C}\right) / \delta\left({ }^{1} \mathrm{H}\right)=186.0 / 7.20,7.14$ (ipso-C of ${ }^{\mathrm{a}} \mathrm{Ph} / \mathrm{o}-, \mathrm{m}-\mathrm{H}$ of $\left.{ }^{\mathrm{a}} \mathrm{Ph}\right), 181.6 / 7.41,7.15$ (ipso-C of ${ }^{b} \mathrm{Ph} / \mathrm{o}-, \mathrm{m}-\mathrm{H}$ of $\left.{ }^{b} \mathrm{Ph}\right), 136.0 / 7.06$ (o-C of ${ }^{b} \mathrm{Ph} / \mathrm{p}-\mathrm{H}$ of $\left.{ }^{b} \mathrm{Ph}\right), 134.6 / 7.02$ (o-C of ${ }^{\mathrm{a}} \mathrm{Ph} / \mathrm{p}-\mathrm{H}$ of ${ }^{\mathrm{a}} \mathrm{Ph}$ ), $126.2 / 7.41\left(\mathrm{p}-\mathrm{C}\right.$ of ${ }^{\mathrm{b}} \mathrm{Ph} / \mathrm{o}-\mathrm{H}$ of $\left.{ }^{\mathrm{b}} \mathrm{Ph}\right), 125.8 / 7.20\left(\mathrm{p}-\mathrm{C}\right.$ of ${ }^{\mathrm{a}} \mathrm{Ph} / \mathrm{o}-\mathrm{H}$ of $\left.{ }^{\mathrm{a}} \mathrm{Ph}\right), 136.9 / 6.31,5.55,5.29$, 1.88, 1.70, 1.40 (C3 / 4-H, 5-H, 2-H, 6-H, 6-H', 7-H), 123.9 / 6.46, 5.54, 5.48 (C4' / 3-H', 2-H', 5-H'), 122.6 / 5.55, 5.29, 1.88, 1.70 (C4 / 5-H, 2-H, 6-H, 6-H'), 121.5 / 6.34, 5.54, 5.48 (C3' / 4-H', 2-H', 5H'), 114.3 / 5.48 (C2' / 5-H'), 114.2 / 6.31, 5.55, 1.88, 1.70 (C2 / 4-H, 5-H, 6-H, 6-H'), 113.8 / 5.29 (C5 / 2-H), 112.7 / 6.46, 5.54 (C5' / 3-H', 2-H'), 101.1 / 6.31, 5.55, 5.29, 0.23 (C1 / 4-H, 5-H, 2-H, $\left.\mathrm{Si}\left(\mathrm{CH}_{3}\right)_{2}\right), 100.3$ / 5.48, 5.54, $0.23\left(\mathrm{Cl}^{\prime}\right.$ / 5-H', 2-H', $\left.\mathrm{Si}\left(\mathrm{CH}_{3}\right)_{2}\right), 32.3$ / 1.62, 1.40 (C6 / 8-H, 7-H), 31.8 / 
1.88, 1.70, $1.40(\mathrm{C} 8 / 6-\mathrm{H}, 7-\mathrm{H}), 26.9$ / 1.88, 1.70, $1.62(\mathrm{C} 7 / 6-\mathrm{H}, 8-\mathrm{H}),-5.8 / 0.23\left(\mathrm{Si}_{\left(\mathrm{CH}_{3}\right.}\right)_{2} /$ $\left.\mathrm{Si}\left(\mathrm{CH}_{3}\right)_{2}\right)$.

Reaction of complex 8 with $\mathrm{HB}\left(\mathrm{C}_{6} \mathrm{~F}_{5}\right)_{2}$, formation of 14 and preparation of $15 .{ }^{1} \mathrm{H}-\mathrm{NMR}$ (toluene$\left.\mathrm{d}_{8}, 600 \mathrm{MHz}\right): \delta=7.21,7.20,7.04$ (each m, 5H, o-, m-, p-H of

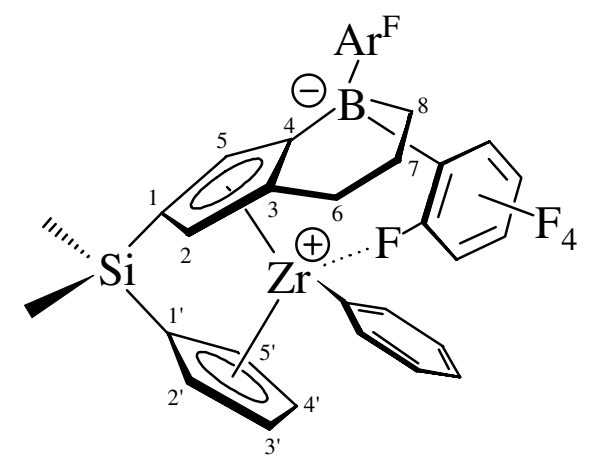
$\mathrm{Ph}), 6.44\left(\mathrm{~m}, 2 \mathrm{H}, 4-\mathrm{H}^{\prime}, 5-\mathrm{H}\right), 6.35\left(\mathrm{~m}, 1 \mathrm{H}, 5-\mathrm{H}^{\prime}\right), 5.95(\mathrm{~m}, 1 \mathrm{H}, 3-$ H'), $5.80(\mathrm{~m}, 1 \mathrm{H}, 2-\mathrm{H}), 5.79\left(\mathrm{~m}, 1 \mathrm{H}, 2-\mathrm{H}^{\prime}\right), 2.32 / 1.68$ (each m, each 1H, 6-H, 6-H'), 1.56/1.23 (each m, each 1H, 8-H, 8- $\mathrm{H}^{\prime}$ ), 1.46/1.30 (each m, each 1H, 7-H, 7-H'), 0.65/0.61 (each s, each $\left.3 \mathrm{H}, \mathrm{Si}\left(\mathrm{CH}_{3}\right)_{2}\right) .{ }^{13} \mathrm{C}\left\{{ }^{1} \mathrm{H}\right\}-\mathrm{NMR}$ (toluene-d $\mathrm{d}_{8}, 150 \mathrm{MHz}$ ): $\delta=189.3$,

128.6, 128.3, 127.6 (ipso-, o-, m-, p-C of Ph), 151.9 (C3), 125.0 (C5'), 121.6 (C4'), 119.8 (C5), 118.8 (C2), 115.2 (C2'), 112.4 (C3'), 105.0 (C1), 99.9 (C1'), 27.8 (C6), 24.3 (C8), 22.8 (C7), -5.5/-5.6 $\left(\mathrm{Si}\left(\mathrm{CH}_{3}\right)_{2}\right) .{ }^{19} \mathrm{~F}-\mathrm{NMR}$ (toluene- $\left.\mathrm{d}_{8}, 564 \mathrm{MHz}\right): \delta=-130.3\left(\mathrm{br}, 4 \mathrm{~F}\right.$, o-F of $\left.\mathrm{C}_{6} \mathrm{~F}_{5}\right),-156.1$, (br, 2F, p-F of $\left.\mathrm{C}_{6} \mathrm{~F}_{5}\right),-162.6\left(\mathrm{br}, 4 \mathrm{~F}, \mathrm{~m}-\mathrm{F}\right.$ of $\left.\mathrm{C}_{6} \mathrm{~F}_{5}\right) .1 \mathrm{D}-\mathrm{TOCSY}\left(\right.$ toluene- $\left.\mathrm{d}_{8}, 600 / 600 \mathrm{MHz}\right): \delta\left({ }^{1} \mathrm{H}\right)_{\text {irr. }} / \delta\left({ }^{1} \mathrm{H}\right)_{\text {resp. }}=7.06$ / 7.21, 7.20 (p-H of Ph / o-, m-H of Ph), 5.95 / 6.44, 6.35, 5.79 (3-H' / 4-H', 5-H', 2-H'), 6.44 / 5.80 (5-H / 2-H), 2.32 / 1.68, 1.56, 1.46, 1.30, 1.23 (6-H / 6-H', 8-H, 7-H, 7-H', 8-H'). NOE-Diff. (toluene$\left.\mathrm{d}_{8}, 600 / 600 \mathrm{MHz}\right): \delta\left({ }^{1} \mathrm{H}\right)_{\text {ir. }} / \delta\left({ }^{1} \mathrm{H}\right)_{\text {resp. }}=2.32 / 5.80,1.68\left(6-\mathrm{H} / 2-\mathrm{H}, 6-\mathrm{H}^{\prime}\right), 0.65$ / 6.44, $6.35\left(\mathrm{Si}\left(\mathrm{CH}_{3}\right)_{2}\right.$

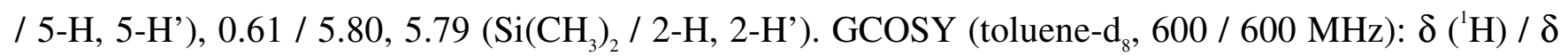
$\left({ }^{1} \mathrm{H}\right)=7.21 / 7.20(\mathrm{o}-\mathrm{H}$ of $\mathrm{Ph} / \mathrm{m}-\mathrm{H}$ of Ph), 7.20 / 7.21, 7.04 (m-H of Ph / o-H of Ph, p-H of Ph), 7.04 / 7.20 (p-H of Ph / m-H of Ph), 6.44 / 6.35, 5.95, 5.79 (4-H' / 5-H', 3-H', 2-H'), 6.44 / 5.80 (5-H / 2-H), 6.35 / 6.44, 5.95, 5.79 (5-H' / 4-H', 3-H', 2-H'), 5.95 / 6.44, 6.35, 5.79 (3-H' / 4-H', 5-H', 2-H'), 5.80 / 6.44 (2-H / 5-H), 5.79 / 6.35, 5.95 (2-H' / 5-H', 3-H'), 2.32 / 1.68, 1.46 (6-H / 6-H', 7-H), 1.68 / 2.32, 
1.46 (6-H’ / 6-H, 7-H), 1.56 / 1.23 (8-H / 8-H'), 1.46 / 2.32, 1.68, 1.23 (7-H / 6-H, 6-H', 8-H'), 1.30 /

2.32, 1.46 (7-H' / 6-H, 7-H), 1.23 / 1.56 (8-H' / 8-H). GHSQC (toluene-d, 150 / $600 \mathrm{MHz}): \delta\left({ }^{13} \mathrm{C}\right) / \delta$ $\left({ }^{1} \mathrm{H}\right)=128.6 / 7.21(\mathrm{o}-\mathrm{C}$ of $\mathrm{Ph} / \mathrm{o}-\mathrm{H}$ of Ph), $128.3 / 7.21(\mathrm{~m}-\mathrm{C}$ of Ph/ m-H of Ph), 127.6 / 7.04 (p-C of Ph/ p-H of Ph), 125.0 / 6.35 (C5' / 5-H'), 121.6 / 6.44 (C4' / 4-H'), 119.8 / 6.44 (C5 / 5-H), 118.8 / 5.80 (C2 / 2-H), 115.2 / 5.79 (C2' / 2-H'), 112.4 / 5.95 (C3' / 3-H'), 27.8 / 2.32, 1.68 (C6 / 6-H, 6-H'), 24.3 / 1.56, 1.23 (C8 / 8-H, 8-H'), 22.8 / 1.46, 1.30 (C7 / 7-H, 7-H'), -5.5/ $0.65\left(\mathrm{Si}\left(\mathrm{CH}_{3}\right)_{2} / \mathrm{Si}\left(\mathrm{CH}_{3}\right)_{2}\right),-$ $5.6 / 0.61\left(\mathrm{Si}\left(\mathrm{CH}_{3}\right)_{2} / \mathrm{Si}\left(\mathrm{CH}_{3}\right)_{2}\right) . \mathrm{GHMBC}\left(\right.$ toluene-d $\left.\mathrm{d}_{8}, 150 / 600 \mathrm{MHz}\right): \delta\left({ }^{13} \mathrm{C}\right) / \delta\left({ }^{1} \mathrm{H}\right)=189.3 / 7.21$ (ipso-C of Ph / o-H of Ph), 151.9 / 6.44, 5.80, 2.32 (C3 / 5-H, 2-H, 6-H), 128.6 / 7.21, 7.04 (o-C of Ph / o-H of Ph, p-H of Ph), 128.3 / 7.20 (m-C of Ph / m-H of Ph), 125.0 / 6.44, 5.95, 5.79 (C5' / 4-H', 3-H', 2-H'), 121.6 / 6.35, 5.95, 5.79 (C3' / 5-H', 4-H', 2-H'), 119.8 / 5.80 (C5 / 2-H), 118.8 / 6.44, 2.32 (C2 / 5-H, 6-H), 115.2 / 6.44, 5.95 (C2' / 4-H', 3-H'), 112.4 / 6.35, 5.79 (C3' / 5-H', 2-H'), 105.0 / 6.44, 5.80, 0.65, 0.61 (C1 / 5-H, 2-H, Si $\left.\left(\mathrm{CH}_{3}\right)_{2}\right), 99.9$ / 6.44, 6.35, 5.79, 0.65, 0.61 (C1' / 4-H', 5-H', 2-H', $\left.\mathrm{Si}\left(\mathrm{CH}_{3}\right)_{2}\right), 27.8$ / 1.23 (C6 / 8-H'), 24.3 / 1.46 (C8 / 7-H), 22.8 / 2.32, 1.23 (C7 / 6-H, 8-H'), -5.5 / 0.61 $\left(\mathrm{Si}\left(\mathrm{CH}_{3}\right)_{2} / \mathrm{Si}\left(\mathrm{CH}_{3}\right)_{2}\right),-5.6 / 0.65\left(\mathrm{Si}\left(\mathrm{CH}_{3}\right)_{2} / \mathrm{Si}\left(\mathrm{CH}_{3}\right)_{2}\right) .{ }^{19} \mathrm{~F}-\mathrm{NMR}$ (toluene-d $\left.{ }_{8}, 564 \mathrm{MHz}, 353 \mathrm{~K}\right): \delta=-$ 116.6 (br, 2F, o-F of $\mathrm{C}_{6} \mathrm{~F}_{5}$ ), -130.0, (br, 2F, o-F' of $\left.\mathrm{C}_{6} \mathrm{~F}_{5}\right),-153.2$ (br, 1F, p-F' of $\left.\mathrm{C}_{6} \mathrm{~F}_{5}\right),-156.0$, (br, 1F, p-F of $\mathrm{C}_{6} \mathrm{~F}_{5}$ ), -161.3 (br, 2F, m-F of $\mathrm{C}_{6} \mathrm{~F}_{5}$ ), -162.7, (br, 2F, m-F' of $\mathrm{C}_{6} \mathrm{~F}_{5}$ ). ${ }^{19} \mathrm{~F} /{ }^{19} \mathrm{~F}$ GCOSY (toluene- $\mathrm{d}_{8}$, 564 / $564 \mathrm{MHz}, 353 \mathrm{~K}): \delta\left({ }^{19} \mathrm{~F}\right) / \delta\left({ }^{19} \mathrm{~F}\right)=-130.0 /-162.7\left(\mathrm{o}-\mathrm{F}^{\prime} / \mathrm{m}-\mathrm{F}^{\prime}\right),-153.2 /-162.7,\left(\mathrm{p}-\mathrm{F}^{\prime} / \mathrm{m}-\mathrm{F}^{\prime}\right),-$ 156.0 / -161.3 (p-F / m-F), -161.3 / -156.0 (m-F / p-F), -162.7 / -130.0, -153.2 (m-F' / o-F', p-F'). The temperature dependent ${ }^{19} \mathrm{~F}$ NMR spectra revealed a conformational equilibration between $14 \mathrm{~A}$ and 14B. Conformer 14A: ${ }^{19} \mathrm{~F}$ NMR ( $\mathrm{d}_{8}$-toluene, $\left.564 \mathrm{MHz}, 203 \mathrm{~K}\right):{ }^{19} \mathrm{~F}-\mathrm{NMR}$ (toluene- $\left.\mathrm{d}_{8}, 564 \mathrm{MHz}, 203 \mathrm{~K}\right)$ : $\delta=-126.2,-175.4$ (each br, each $1 \mathrm{~F}$, o-F of $\mathrm{C}_{6} \mathrm{~F}_{5}$ ), $-131.2,-132.1$ (br, $2 \mathrm{~F}, \mathrm{o}-\mathrm{F}$ ' of $\left.\mathrm{C}_{6} \mathrm{~F}_{5}\right),-155.6,-$ 164.2 (each m, each 1F, m-F of $\mathrm{C}_{6} \mathrm{~F}_{5}$ ), -156.8 (br, $1 \mathrm{~F}, \mathrm{p}-\mathrm{F}$ of $\mathrm{C}_{6} \mathrm{~F}_{5}$ ), -160.0 (br, 1F, p-F' of $\mathrm{C}_{6} \mathrm{~F}_{5}$ ), 162.8, -164.6 (each m, each 1F, m-F' of $\mathrm{C}_{6} \mathrm{~F}_{5}$ ). ${ }^{19} \mathrm{~F} /{ }^{19} \mathrm{~F}$ GCOSY (toluene- $\left.\mathrm{d}_{8}, 564 / 564 \mathrm{MHz}, 203 \mathrm{~K}\right)$ : $\delta\left({ }^{19} \mathrm{~F}\right) / \delta\left({ }^{19} \mathrm{~F}\right)=-126.2 /-164.2(\mathrm{o}-\mathrm{F} / \mathrm{m}-\mathrm{F}),-131.2 /-164.6\left(\mathrm{o}-\mathrm{F}^{\prime} / \mathrm{m}-\mathrm{F}^{\prime}\right),-132.1 /-162.8$ (o-F' / m-F'), -155.6 / -175.4, -156.8 (m-F / o-F, p-F), -156.8 / -155.6, -164.2 (p-F / m-F), -160.0 / -162.8, 
-164.6 (p-F' / m-F'), -162.8 / -132.1, -160.0 (m-F' / o-F', p-F'), -164.2 / -126.2, -156.8 (m-F / o-F, p-F), -164.6 / -131.2, -160.0 (m-F' / o-F', p-F'), -175.4 / -155.6 (o-F / m-F). Conformer B: ${ }^{19}$ F-NMR (toluene-d $\left.\mathrm{d}_{8}, 564 \mathrm{MHz}, 203 \mathrm{~K}\right): \delta=-108.6,-111.2\left(\right.$ each br, each 1F, o-F of $\mathrm{C}_{6} \mathrm{~F}_{5}$ ), -129.6, (br, 2F, o-F' of $\mathrm{C}_{6} \mathrm{~F}_{5}$ ), -150.0 (br, 1F, p-F' of $\mathrm{C}_{6} \mathrm{~F}_{5}$ ), -154.7 (br, 1F, p-F of $\mathrm{C}_{6} \mathrm{~F}_{5}$ ), -160.0, -161.5 (each m, each 1F, m-F of $\mathrm{C}_{6} \mathrm{~F}_{5}$ ), -161.1 (each br, each $1 \mathrm{~F}, \mathrm{~m}-\mathrm{F}$ ' of $\mathrm{C}_{6} \mathrm{~F}_{5}$ ). ${ }^{19} \mathrm{~F} /{ }^{19} \mathrm{~F}$ GCOSY (toluene-d $\mathrm{d}_{8}, 564$ / $564 \mathrm{MHz}$, $203 \mathrm{~K}): \delta\left({ }^{19} \mathrm{~F}\right) / \delta\left({ }^{19} \mathrm{~F}\right)=-108.6 /-160.0(\mathrm{o}-\mathrm{F} / \mathrm{m}-\mathrm{F}),-111.2 /-161.5(\mathrm{o}-\mathrm{F} / \mathrm{m}-\mathrm{F}),-129.6 /-161.1(\mathrm{o}-$ F' / m-F'), -150.0 / -161.1 (p-F' / m-F'), -154.7 / -160.0, -161.5 (p-F / m-F), -160.0 / -108.6, -154.7 (m-F / o-F, p-F), -161.1 / -129.6 , -150.0 (m-F' / o-F', p-F'), -161.5 / -111.2, -154.7 (m-F / o-F, p-F).

15 as a yellow solid, mp $168^{\circ} \mathrm{C}$. Anal. Calcd. for $\mathrm{C}_{36} \mathrm{H}_{32} \mathrm{BF}_{10} \mathrm{PSiZr}$ (815.7): C 53.01, $\mathrm{H}$ 3.95; found C 53.53, H 3.69\%. IR (KBr): $\tilde{v}=2917(\mathrm{~m}), 1630(\mathrm{~m}), 1504(\mathrm{~s}), 1435$ (s),

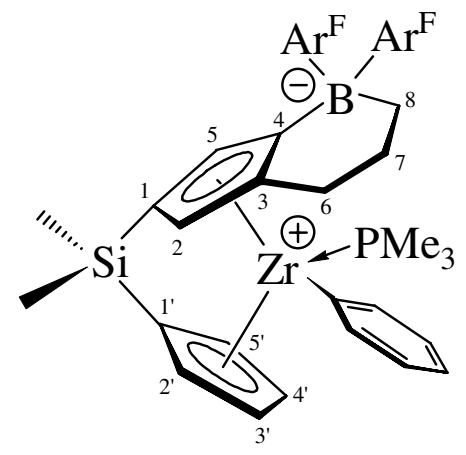
1260 (s), 1078 (s), 1043 (s), 966 (s), 932 (s), 819 (s), 722 (w), 694 (m), $666(\mathrm{~m}), 463(\mathrm{w}) \mathrm{cm}^{-1} .{ }^{1} \mathrm{H}-\mathrm{NMR}$ (toluene-d $\left.{ }_{8}, 600 \mathrm{MHz}\right): \delta=7.15,7.12$, 7.00 (each m, 5H, m-, o-, p-H of Ph), 6.71 (m, 1H, 4-H'), 6.09 (m, 1H, 5-H), 5.97 (m, 1H, 3-H'), 5.79 (m, 1H, 2-H), 5.74 (m, 1H, 5-H'), 5.53 (m, 1H, 2-H'), 2.30/1.96 (each m, each 1H, 6-H, 6-H'), 1.66/0.96 (each m, each 1H, 7-H, 7-H'), 1.31/0.85 (each m, each 1H, 8-H, 8-H'), 0.35/0.31 (each s, each 3H, $\left.\mathrm{Si}\left(\mathrm{CH}_{3}\right)_{2}\right)$, $0.22\left(\mathrm{~d},{ }^{2} \mathrm{~J}_{\mathrm{PH}}=10.1 \mathrm{~Hz}, 9 \mathrm{H}, \mathrm{PMe}_{3}\right) .{ }^{13} \mathrm{C}\left\{{ }^{1} \mathrm{H}\right\}-\mathrm{NMR}$ (toluene-d $\left.\mathrm{d}_{8}, 150 \mathrm{MHz}\right): \delta=186.7,131.4,127.0$, 126.0 (ipso-, o-, m-, p-C of Ph), 144.3 (C3), 125.0 (C3'), 120.2 (C4'), 119.4 (C2), 116.3 (C5), 115.4 (C2’), 109.6 (C5'), 99.7 (C1), 97.4 (C1'), 28.5 (C6), $24.3(\mathrm{C} 7), 16.2(\mathrm{C} 8), 8.9$ (d, ${ }^{1} \mathrm{~J}_{\mathrm{CP}}=30.2 \mathrm{~Hz}$, $\left.\mathrm{PMe}_{3}\right),-4.9 /-5.8\left(\mathrm{Si}\left(\mathrm{CH}_{3}\right)_{2}\right) ; 149.0\left({ }^{1} \mathrm{~J}_{\mathrm{CF}}=238.3 \mathrm{~Hz}, \mathrm{o}-\mathrm{C}\right.$ of $\left.\mathrm{C}_{6} \mathrm{~F}_{5}\right), 139.5\left({ }^{1} \mathrm{~J}_{\mathrm{CF}}=261.6 \mathrm{~Hz}, \mathrm{p}-\mathrm{C}\right.$ of $\left.\mathrm{C}_{6} \mathrm{~F}_{5}\right)$, $137.7\left({ }^{1} \mathrm{~J}_{\mathrm{CF}}=263.0 \mathrm{~Hz}, \mathrm{~m}-\mathrm{C}\right.$ of $\left.\mathrm{C}_{6} \mathrm{~F}_{5}\right) .{ }^{11} \mathrm{~B}\left\{{ }^{1} \mathrm{H}\right\}-\mathrm{NMR}$ (toluene-d $\left.\mathrm{d}_{8}, 64 \mathrm{MHz}\right): \delta=-13.0\left(\mathrm{v}_{1 / 2}=224 \mathrm{~Hz}\right)$. ${ }^{31} \mathrm{P}\left\{{ }^{1} \mathrm{H}\right\}-\mathrm{NMR}$ (toluene-d $\left.\mathrm{d}_{8}, 81 \mathrm{MHz}\right): \delta=-9.4\left(\mathrm{v}_{1 / 2}=0.3 \mathrm{~Hz}\right) .{ }^{19} \mathrm{~F}-\mathrm{NMR}$ (toluene-d $\left.\mathrm{d}_{8}, 564 \mathrm{MHz}\right): \delta=-$ 106.3, -109.5 (each br, each 1F, o-F of $\left.\mathrm{C}_{6} \mathrm{~F}_{5}\right),-124.9\left(\mathrm{br}, 2 \mathrm{~F}\right.$, o-F of $\left.\mathrm{C}_{6} \mathrm{~F}_{5}\right),-156.9,-157.8\left(\right.$ each t, ${ }^{3} \mathrm{~J}_{\mathrm{FF}}$ $=20.1 \mathrm{~Hz}$, each 1F, p-F of $\left.\mathrm{C}_{6} \mathrm{~F}_{5}\right),-161.9,-162.2\left(\right.$ each $\mathrm{m}$, each $1 \mathrm{~F}, \mathrm{~m}-\mathrm{F}$ of $\left.\mathrm{C}_{6} \mathrm{~F}_{5}\right),-163.1(\mathrm{~m}, 2 \mathrm{~F}, \mathrm{~m}-\mathrm{F}$ 
of $\left.\mathrm{C}_{6} \mathrm{~F}_{5}\right) .1 \mathrm{D}-\mathrm{TOCSY}$ (toluene-d $\left.{ }_{8}, 600 / 600 \mathrm{MHz}\right): \delta\left({ }^{1} \mathrm{H}\right)_{\text {irr. }} / \delta\left({ }^{1} \mathrm{H}\right)_{\text {resp. }}=7.00 / 7.15,7.12(\mathrm{p}-\mathrm{H}$ of $\mathrm{Ph} /$ m-, o-H of Ph), 5.74 / 6.71, 5.97, 5.53 (5-H' / 4-H', 3-H', 2-H'), 5.79 / 6.09 (2-H / 5-H), 2.30 / 1.96 , 1.66, 1.31, 0.96, 0.85 (6-H / 6-H', 7-H, 8-H, 7-H', 8-H'). 1D-ROESY (toluene-d, 600 / 600 MHz): $\delta$ $\left({ }^{1} \mathrm{H}\right)_{\text {irr. }} / \delta\left({ }^{1} \mathrm{H}\right)_{\text {resp. }}=7.12 / 7.15,5.97,5.79,5.53,0.22\left(\mathrm{o}-\mathrm{H}\right.$ of $\mathrm{Ph} / \mathrm{m}-\mathrm{H}$ of $\left.\mathrm{Ph}, 3-\mathrm{H}^{\prime}, 2-\mathrm{H}, 2-\mathrm{H}^{\prime}, \mathrm{PMe}_{3}\right)$, 6.71 / 5.97, 5.74, 0.22 (4-H' / 3-H', 5-H', $\left.\mathrm{PMe}_{3}\right), 6.09$ / 5.74, 0.35, 0.22 (5-H / 5-H', $\left.\mathrm{Si}\left(\mathrm{CH}_{3}\right)_{2}, \mathrm{PMe}_{3}\right)$, 5.97 / 7.12, 6.71, 5.53 (3-H' / o-H of Ph, 4-H', 2-H'), 5.79 / 7.12, 5.97, 5.53, 2.30, 0.31 (2-H / o-H of Ph, 3-H', 2-H', 6-H, Si( $\left.\left(\mathrm{CH}_{3}\right)_{2}\right)$, 5.74 / 6.71, 6.09, 0.35 (5-H' / 4-H', 5-H, $\left.\mathrm{Si}\left(\mathrm{CH}_{3}\right)_{2}\right), 5.53$ / 7.12, 5.79, 0.31 (2-H' / o-H of Ph, 2-H, Si( $\left.\left(\mathrm{CH}_{3}\right)_{2}\right), 2.30$ / 7.12, 5.79, 1.96, 1.66, 0.96 (6-H / o-H of Ph, 2-H, 6-H', 7-H, 7-H'), 1.96 / 7.12, 2.30, 1.66, 1.31 (6-H' / o-H of Ph, 6-H, 7-H, 8-H), 1.66 / 2.30, 1.96, 1.31, 0.96, 0.85 (7-H / 6-H, 6-H’, 8-H, 7-H’, 8-H’), 1.31 / 0.85, 0.22 (8-H / 8-H’, $\mathrm{PMe}_{3}$ ), 0.96 / 2.30, 1.66, 0.85, 0.22 (7-H' / 6-H, 7-H, 8-H', $\mathrm{PMe}_{3}$ ), 0.85 / 1.66, 1.31, 0.22 (8-H’ / 7-H, 8-H, $\mathrm{PMe}_{3}$ ), 0.35/ 6.09, 5.74 $\left(\mathrm{Si}\left(\mathrm{CH}_{3}\right)_{2} / 5-\mathrm{H}, 5-\mathrm{H}^{\prime}\right), 0.31$ / 5.79, $5.53\left(\mathrm{Si}\left(\mathrm{CH}_{3}\right)_{2} / 2-\mathrm{H}, 2-\mathrm{H}^{\prime}\right) 0.22$ / 6.09, 0.96, $0.85\left(\mathrm{PMe}_{3} / 5-\mathrm{H}, 7-\right.$ H', 8-H'). GCOSY (toluene-d $\left.\mathrm{d}_{8}, 600 / 600 \mathrm{MHz}\right): \delta\left({ }^{1} \mathrm{H}\right) / \delta\left({ }^{1} \mathrm{H}\right)=7.15 / 7.12,7.00(\mathrm{~m}-\mathrm{H}$ of $\mathrm{Ph} / \mathrm{o}-\mathrm{H}$ of $\mathrm{Ph}, \mathrm{p}-\mathrm{H}$ of $\mathrm{Ph}), 7.12$ / 7.15 (o-H of $\mathrm{Ph} / \mathrm{m}-\mathrm{H}$ of $\mathrm{Ph}$ ), 7.00 / 7.15 (p-H of $\mathrm{Ph} / \mathrm{m}-\mathrm{H}$ of $\mathrm{Ph}$ ), 6.71 / 5.97, 5.74, 5.53 (4-H' / 3-H', 5-H', 2-H’), 6.09 / 5.79 (5-H / 2-H), 5.97 / 6.71, 5.74, 5.53 (3-H’ / 4-H', 5-H', 2-H'), 5.79 / 6.09 (2-H / 5-H), 5.74 / 6.71, 5.97, 5.53 (5-H' / 4-H', 3-H', 2-H'), 5.53 / 5.97, 5.74 (2-H' / 3-H', 5-H'), 2.30 / 1.96, 1.66, 0.96 (6-H / 6-H', 7-H, 7-H'), 1.96 / 2.30, 1.66, 0.96 (6-H' / 6-H, 7-H, 7H'), 1.66 / 2.30, 1.96, 1.66, 0.96, 0.85 (7-H / 6-H, 6-H', 7-H', 8-H'), 0.96 / 2.30, 1.96, 1.66 (7-H' / 6H, 6-H', 7-H), 0.85 / 1.66 (8-H' / 7-H). GHSQC (toluene-d, $150 / 600 \mathrm{MHz}): \delta\left({ }^{13} \mathrm{C}\right) / \delta\left({ }^{1} \mathrm{H}\right)=131.4 /$ 7.12 (o-C of $\mathrm{Ph} / \mathrm{o}-\mathrm{H}$ of $\mathrm{Ph}), 127.0$ / 7.15 (m-C of $\mathrm{Ph} / \mathrm{m}-\mathrm{H}$ of $\mathrm{Ph}), 126.0 / 7.00$ (p-C of $\mathrm{Ph} / \mathrm{p}-\mathrm{H}$ of Ph), 125.0 / 5.97 (C3’ / 3-H'), 120.2 / 6.71 (C4' / 4-H'), 119.4 / 5.79 (C2 / 2-H), 116.3 / 6.09 (C5 / 5 H), 115.4 / 5.53 (C2’ / 2-H'), 109.6 / 5.74 (C5’ / 5-H'), 28.5 / 2.30, 1.96 (C6 / 6-H, 6-H'), 24.3 / 1.66, 0.96 (C7 / 7-H, 7-H'), 16.2 / 1.31, 0.85 (C8 / 8-H, 8-H'), 8.9 / $0.22\left(\mathrm{PMe}_{3} / \mathrm{PMe}_{3}\right),-4.9 / 0.35\left(\mathrm{Si}_{(}\left(\mathrm{CH}_{3}\right)_{2}\right.$ / $\left.\mathrm{Si}\left(\mathrm{CH}_{3}\right)_{2}\right),-5.8 / 0.31\left(\mathrm{Si}\left(\mathrm{CH}_{3}\right)_{2} / \mathrm{Si}\left(\mathrm{CH}_{3}\right)_{2}\right)$. GHMBC (toluene-d $\left.\mathrm{d}_{8}, 150 / 600 \mathrm{MHz}\right): \delta\left({ }^{13} \mathrm{C}\right) / \delta\left({ }^{1} \mathrm{H}\right)=$ 186.7 / 7.15, 7.12 (ipso-C of Ph / m-, o-H of Ph), 144.3 / 6.09, 5.79, 2.30, 1.96 (C3 / 5-H, 2-H, 6-H, 6- 
H'), 131.4 / 7.12, 7.00 (o-C of Ph / o-, p-H of Ph), 127.0 / 7.15, 7.00 (o-C of Ph / o-, p-H of Ph), 126.0 / 7.12 (p-C of Ph / o-H of Ph), 125.0 / 5.74, 5.53 (C3' / 5-H', 2-H'), 120.2 / 5.74, 5.53 (C4' / 5-H', 2H'), 119.4 / 6.09, 2.30, 1.96 (C2 / 5-H, 6-H, 6-H'), 116.3 / 5.79 (C5 / 2-H), 115.4 / 6.71, 5.74 (C2' / 4H', 5-H'), 109.6 / 5.97, 5.53 (C5' / 3-H', 2-H'), 99.7 / 6.09, 5.79, 0.35, 0.31 (C1 / 5-H, 2-H, Si( $\left.\left(\mathrm{CH}_{3}\right)_{2}\right)$, 97.4 / 5.74, 5.53, 0.35, 0.31 (C1' / 5-H', 2-H', $\left.\mathrm{Si}\left(\mathrm{CH}_{3}\right)_{2}\right), 24.3$ / 2.30, 1.96 (C7 / 6-H, 6-H'), 8.9 / 0.22 $\left(\mathrm{PMe}_{3} / \mathrm{PMe}_{3}\right),-4.9 / 0.31\left(\mathrm{Si}\left(\mathrm{CH}_{3}\right)_{2} / \mathrm{Si}\left(\mathrm{CH}_{3}\right)_{2}\right),-5.8 / 0.35\left(\mathrm{Si}\left(\mathrm{CH}_{3}\right)_{2} / \mathrm{Si}\left(\mathrm{CH}_{3}\right)_{2}\right)$.

NMR data of 15 at $213 \mathrm{~K}:{ }^{1} \mathrm{H}-\mathrm{NMR}$ (toluene-d $\left.{ }_{8}, 600 \mathrm{MHz}, 213 \mathrm{~K}\right): \delta=7.24,7.10$ (each m, 5H, m-, o, p-H of Ph), 6.82 (m, 1H, 4-H'), 6.06 (m, 1H, 5-H), 5.87 (m, 1H, 3-H'), 5.83 (m, 1H, 2-H), 5.79 (m, 1H, 5-H'), 5.49 (m, 1H, 2-H'), 2.38/2.04 (each m, each 1H, 6-H, 6-H'), 1.63/0.88 (each m, each 1H, 7H, 7-H'), 1.38/0.97 (each m, each 1H, 8-H, 8-H'), 0.26/0.22 (each s, each 3H, Si $\left.\left(\mathrm{CH}_{3}\right)_{2}\right), 0.02(\mathrm{~d}$, ${ }^{2} \mathrm{~J}_{\mathrm{PH}}=10.4 \mathrm{~Hz}, 9 \mathrm{H}, \mathrm{PMe}_{3}$ ). ${ }^{13} \mathrm{C}\left\{{ }^{1} \mathrm{H}\right\}-\mathrm{NMR}$ (toluene-d $, 150 \mathrm{MHz}, 213 \mathrm{~K}$ ): $\delta=186.1,126.7,125.7$ (ipso-, o-, m-, p-C of Ph), 142.9 (C3), 137.0 (C4), 124.8 (C3’), 120.6 (C4'), 120.0 (C2), 115.5 (C2'), 115.2 (C5), 108.4 (C5'), 99.4 (C1), 96.6 (C1'), 28.4 (C6), 24.1 (C7), 15.8 (C8), 7.6 (d, ${ }^{1} \mathrm{~J}_{\mathrm{CP}}=32.0 \mathrm{~Hz}, \mathrm{PMe}_{3}$ ), $-5.3 /-6.5\left(\mathrm{Si}\left(\mathrm{CH}_{3}\right)_{2}\right) .{ }^{19} \mathrm{~F}-\mathrm{NMR}\left(\right.$ toluene-d $\left.\mathrm{d}_{8}, 564 \mathrm{MHz}, 213 \mathrm{~K}\right): \delta=-105.0,-110.0($ each br, each $1 \mathrm{~F}, \mathrm{o}-\mathrm{F}$ of $\mathrm{C}_{6} \mathrm{~F}_{5}$ ), $-120.7,-129.8$ (br, $2 \mathrm{~F}$, o-F' of $\mathrm{C}_{6} \mathrm{~F}_{5}$ ), -157.4 (br, 1F, p-F of $\mathrm{C}_{6} \mathrm{~F}_{5}$ ), -157.8 (br, 1F, p-F' of $\mathrm{C}_{6} \mathrm{~F}_{5}$ ), $-162.6,-162.7$ (each m, each 1F, m-F of $\mathrm{C}_{6} \mathrm{~F}_{5}$ ), -162.8, -164.2 (each m, each 1F, m-F' of $\mathrm{C}_{6} \mathrm{~F}_{5}$ ). 1D-TOCSY (toluene-d $\left.\mathrm{d}_{8}, 600 / 600 \mathrm{MHz}, 213 \mathrm{~K}\right): \delta\left({ }^{1} \mathrm{H}\right)_{\text {irr. }} / \delta\left({ }^{1} \mathrm{H}\right)_{\text {resp. }}=7.24 / 7.10(\mathrm{H}$ of $\mathrm{Ph} / \mathrm{H}$ of Ph$)$, 5.49 / 6.82, 5.87, 5.79 (2-H' / 4-H', 3-H', 5-H'), 6.06 / 5.83 (5-H / 2-H), 2.38 / 2.04, 1.63, 1.38, 0.97, 0.88 (6-H / 6-H', 7-H, 8-H, 8-H', 7-H'). 1D-ROESY (toluene-d $, 600 / 600 \mathrm{MHz}, 213 \mathrm{~K}): \delta\left({ }^{1} \mathrm{H}\right)_{\text {irr. }} / \delta$ $\left({ }^{1} \mathrm{H}\right)_{\text {resp. }}=7.24 / 7.10,5.83(\mathrm{H}$ of $\mathrm{Ph} / \mathrm{H}$ of $\mathrm{Ph}, 2-\mathrm{H}), 6.82$ / 5.87, 5.79, $0.02\left(4-\mathrm{H}^{\prime} / 3-\mathrm{H}^{\prime}, 5-\mathrm{H}^{\prime}, \mathrm{PMe}_{3}\right)$, 6.06 / 5.79, 0.26, 0.02 (5-H / 5-H', $\left.\mathrm{Si}\left(\mathrm{CH}_{3}\right)_{2}, \mathrm{PMe}_{3}\right), 5.87$ / 7.24, 6.82, 5.49 (3-H' / H of $\mathrm{Ph}, 4-\mathrm{H}^{\prime}$, 2-H'), 5.79 / 6.82, 6.06, 5.87, 0.26 (5-H' / 4-H', 5-H, 3-H', $\left.\mathrm{Si}\left(\mathrm{CH}_{3}\right)_{2}\right), 5.49$ / 5.87, 5.83, 0.22 (2-H' / 3H', 2-H, $\left.\mathrm{Si}\left(\mathrm{CH}_{3}\right)_{2}\right), 2.38$ / 7.10, 5.83, 0.88 (6-H / H of Ph, 2-H, 7-H'), 1.63 / 7.10, 0.88 (6-H / H of Ph, 7-H'), 0.26 / 6.06, $5.79\left(\mathrm{Si}\left(\mathrm{CH}_{3}\right)_{2}\right.$ / 5-H, 5-H'), 0.22 / 5.83, $5.49\left(\mathrm{Si}\left(\mathrm{CH}_{3}\right)_{2} / 2-\mathrm{H}, 2-\mathrm{H}^{\prime}\right) 0.02$ / 6.06, 2.38, 0.97, $0.88\left(\mathrm{PMe}_{3} /\right.$ 5-H, 6-H, 8-H', 7-H'). GCOSY (toluene-d $\left.{ }_{8}, 600 / 600 \mathrm{MHz}, 213 \mathrm{~K}\right): \delta\left({ }^{1} \mathrm{H}\right) / \delta\left({ }^{1} \mathrm{H}\right)=$ 
7.24 / 7.10 (H of $\mathrm{Ph} / \mathrm{H}$ of $\mathrm{Ph}), 7.10$ / 7.24 (H of Ph / H of Ph), 6.82 / 5.87, 5.79, 5.49 (4-H' / 3-H', 5-

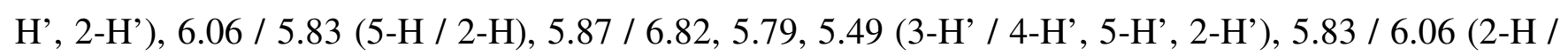

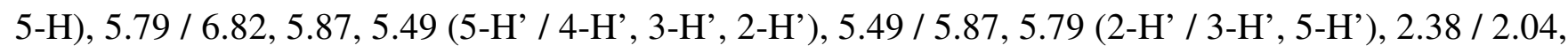
1.63, 0.88 (6-H / 6-H', 7-H, 7-H'), 2.04 / 2.38, 1.63, 0.88 (6-H' / 6-H, 7-H, 7-H'), 1.63 / 2.38, 2.04, 0.97, 0.88 (7-H / 6-H, 6-H', 8-H', 7-H'), 0.88 / 2.38, 2.04, 1.63 (7-H' / 6-H, 6-H’, 7-H), 0.97 / 1.63 (8-H' / 7-H). GHSQC (toluene-d $\left.\mathrm{d}_{8}, 150 / 600 \mathrm{MHz}, 213 \mathrm{~K}\right): \delta\left({ }^{13} \mathrm{C}\right) / \delta\left({ }^{1} \mathrm{H}\right)=126.7 / 7.24(\mathrm{Ph}-\mathrm{C} / \mathrm{H}$ of Ph), 125.7 / 7.10 (Ph-C / H of Ph), 124.8 / 5.87 (C3' / 3-H'), 120.6 / 6.82 (C4' / 4-H'), 120.0 / 5.83 (C2 / 2-H), 115.2 / 6.06 (C5 / 5-H), 115.5 / 5.49 (C2' / 2-H'), 108.4 / 5.79 (C5' / 5-H'), 28.4 / 2.38, 2.04 (C6 / 6-H, 6-H'), 24.1 / 1.63, 0.88 (C7 / 7-H, 7-H'), 15.8 / 1.38, 0.97 (C8 / 8-H, 8-H'), 7.6 / $0.02\left(\mathrm{PMe}_{3}\right.$

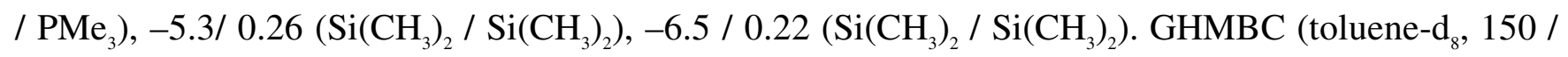
$600 \mathrm{MHz}, 213 \mathrm{~K}): \delta\left({ }^{13} \mathrm{C}\right) / \delta\left({ }^{1} \mathrm{H}\right)=186.1 / 7.24($ ipso-C of $\mathrm{Ph} / \mathrm{H}$ of $\mathrm{Ph}), 142.9 / 6.06,5.83(\mathrm{C} 3 / 5-\mathrm{H}$, 2-H), 137.0 / 6.06, 5.83 (C4 / 5-H, 2-H), 124.8 / 5.79, 5.49 (C3' / 5-H', 2-H'), 120.6 / 5.79 (C4' / 5H’), 120.0 / 6.06 (C2 / 5-H), 115.5 / 5.79 (C2' / 5-H'), 115.2 / 5.83 (C5 / 2-H), 108.4 / 5.49 (C5' / 2H'), 99.4 / 6.06, 5.83, 0.26, $0.22\left(\mathrm{C} 1 / 5-\mathrm{H}, 2-\mathrm{H}, \mathrm{Si}\left(\mathrm{CH}_{3}\right)_{2}\right), 96.6$ / 5.79, 5.49, 0.26, 0.22 (C1' / 5-H', 2-

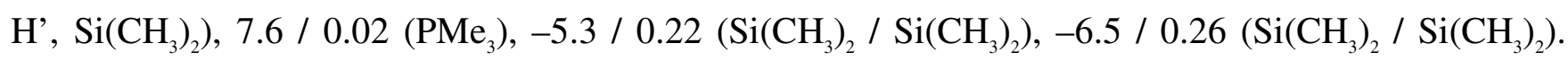
${ }^{19} \mathrm{~F} /{ }^{19} \mathrm{~F}$ GCOSY (toluene- $\left.\mathrm{d}_{8}, 564 / 564 \mathrm{MHz}, 213 \mathrm{~K}\right): \delta\left({ }^{19} \mathrm{~F}\right) / \delta\left({ }^{19} \mathrm{~F}\right)=-105.0 /-162.7(\mathrm{o}-\mathrm{F} / \mathrm{m}-\mathrm{F}),-$ 110.0 / -162.6 (o-F / m-F), -120.7 / -164.2 (o-F' / m-F'), -129.8 / -162.8 (o-F' / m-F'), -157.4 / 162.6, -162.7 (p-F / m-F), -157.8 / -162.8, -164.2 (p-F' / m-F'), -162.6 / -110.0, -157.4 (m-F / o-F, p-F), -162.7 / -105.0, -157.4 (m-F / o-F, p-F), -162.8 / -129.8, -157.8 (m-F' / o-F', p-F'), -164.2 / 120.7, -158.0 (m-F' / o-F', p-F').

\section{Reaction of complex 12 with n-butylisocyanide, preparation of the insertion product $16 . \mathrm{mp}$} $147^{\circ} \mathrm{C}$ (decomp.). Anal. Calcd. for $\mathrm{C}_{49} \mathrm{H}_{47} \mathrm{~N}_{2} \mathrm{BF}_{10} \mathrm{SiZr}$ (984.9): C 59.81, H 4.81, N 2.85; found C 58.87,

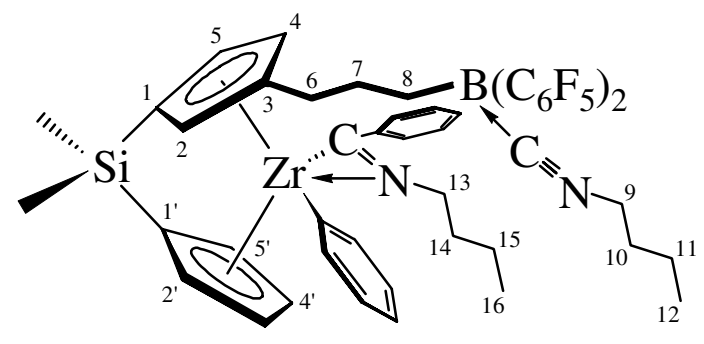
$\mathrm{H} 4.63, \mathrm{~N} 2.68 \%$. IR $(\mathrm{KBr}): \widetilde{v}=2909(\mathrm{~m}), 1637(\mathrm{~m})$, $1497(\mathrm{~s}), 1455(\mathrm{~s}), 1358(\mathrm{w}), 1253(\mathrm{~m}), 1085$ (s), $966(\mathrm{~s})$, 
805 (s), 693 (m), 465 (w) cm ${ }^{-1} .{ }^{1} \mathrm{H}-\mathrm{NMR}$ (benzene-d, $\left.600 \mathrm{MHz}\right): \delta=7.97,7.44,7.28$ (each m, 5H, o-, m-, p-H of $\left.{ }^{\mathrm{a}} \mathrm{Ph}\right), 6.96,7.23,7.07$ (each m, 5H, o-, m-, p-H of $\left.{ }^{b} \mathrm{Ph}\right), 6.52\left(\mathrm{~m}, 1 \mathrm{H}, 3-\mathrm{H}^{\prime}\right), 6.03(\mathrm{~m}, 1 \mathrm{H}, 5-$

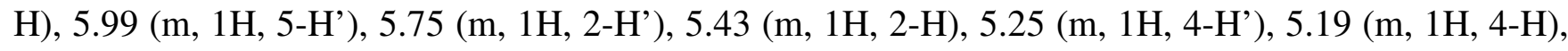
3.58 (m, 2H,13-H), 2.21 (m, 2H, 9-H), 2.04 (m, 2H, 6-H), 1.51/1.30 (each m, each 1H, 14-H, 14-H'), 1.44/1.35 (m, 2H, 7-H, 7-H'), 1.44/1.33 (m, 2H, 8-H, 8-H'), 1.03 (m, 2H, 15-H), 0.78 (m, 2H, 10-H), $0.75(\mathrm{~m}, 2 \mathrm{H}, 11-\mathrm{H}), 0.68\left(\mathrm{t},{ }^{3} \mathrm{~J}_{\mathrm{HH}}=7.3 \mathrm{~Hz}, 3 \mathrm{H}, 16-\mathrm{H}\right), 0.43\left(\mathrm{t},{ }^{3} \mathrm{~J}_{\mathrm{HH}}=7.2 \mathrm{~Hz}, 3 \mathrm{H}, 12-\mathrm{H}\right), 0.40 / 0.26($ each s, each $\left.3 \mathrm{H}, \mathrm{Si}\left(\mathrm{CH}_{3}\right)_{2}\right) .{ }^{13} \mathrm{C}\left\{{ }^{1} \mathrm{H}\right\}-\mathrm{NMR}$ (benzene-d $\left.6,150 \mathrm{MHz}\right): \delta=240.1(\mathrm{Zr}-\mathrm{C}-\mathrm{N}), 179.1,141.8,126.7$, 123.9 (ipso-, o-, m-, p-C of ${ }^{\mathrm{a}} \mathrm{Ph}$ ), 142.6, 129.8, 127.4, 123.1 (ipso-, m-, p-, o-C of ${ }^{\mathrm{b}} \mathrm{Ph}$ ), 138.8 (C3), 121.9 (C3'), 112.1 (C4'), 111.9 (C4), 108.1 (C5), 107.6 (C5'), 105.6 (C2'), 105.4 (C2), 102.3 (C1), 102.0 (C1'), 48.6 (C13), 43.0 (C9), 33.6 (C6), 31.2 (C14), 30.9 (C7), 29.2 (C10), 20.7 (C8), 20.5 (C15), 19.1 (C11), $13.5(\mathrm{C} 16), 12.6(\mathrm{C} 12),-4.4 /-6.3\left(\mathrm{Si}\left(\mathrm{CH}_{3}\right)_{2}\right) ; 148.2\left({ }^{1} \mathrm{~J}_{\mathrm{CF}}=245.7 \mathrm{~Hz}\right.$, o-C of $\left.\mathrm{C}_{6} \mathrm{~F}_{5}\right)$, $139.9\left({ }^{1} \mathrm{~J}_{\mathrm{CF}}=250.1 \mathrm{~Hz}, \mathrm{p}-\mathrm{C}\right.$ of $\left.\mathrm{C}_{6} \mathrm{~F}_{5}\right), 137.6\left({ }^{1} \mathrm{~J}_{\mathrm{CF}}=261.0 \mathrm{~Hz}, \mathrm{~m}-\mathrm{C}\right.$ of $\left.\mathrm{C}_{6} \mathrm{~F}_{5}\right), 117.7$ (ipso-C of $\left.\mathrm{C}_{6} \mathrm{~F}_{5}\right)$. ${ }^{11} \mathrm{~B}\left\{{ }^{1} \mathrm{H}\right\}$-NMR (benzene-d, $\left.64 \mathrm{MHz}\right): \delta=-18.5\left(\mathrm{v}_{1 / 2}=232 \mathrm{~Hz}\right) .{ }^{19} \mathrm{~F}-\mathrm{NMR}$ (benzene-d, $\left.564 \mathrm{MHz}\right)$ : $\delta=-132.8\left(\mathrm{~m}, 4 \mathrm{~F}, \mathrm{o}-\mathrm{F}\right.$ of $\left.\mathrm{C}_{6} \mathrm{~F}_{5}\right),-157.4\left(\mathrm{t},{ }^{3} \mathrm{~J}_{\mathrm{FF}}=20.7 \mathrm{~Hz}, 2 \mathrm{~F}, \mathrm{p}-\mathrm{F}\right.$ of $\left.\mathrm{C}_{6} \mathrm{~F}_{5}\right),-163.2\left(\mathrm{~m}, 4 \mathrm{~F}, \mathrm{~m}-\mathrm{F}\right.$ of $\left.\mathrm{C}_{6} \mathrm{~F}_{5}\right)$. 1D-TOCSY (benzene-d $\left.{ }_{6}, 600 / 600 \mathrm{MHz}\right): \delta\left({ }^{1} \mathrm{H}\right)_{\text {irr. }} / \delta\left({ }^{1} \mathrm{H}\right)_{\text {resp. }}=7.97 / 7.44,7.28\left(\mathrm{o}-\mathrm{H}\right.$ of ${ }^{\mathrm{a}} \mathrm{Ph} / \mathrm{m}-, \mathrm{p}-\mathrm{H}$ of $\left.{ }^{\mathrm{a}} \mathrm{Ph}\right), 6.96$ / 7.23, 7.07 (o-H of ${ }^{\mathrm{b}} \mathrm{Ph} / \mathrm{m}-, \mathrm{p}-\mathrm{H}$ of $\left.{ }^{\mathrm{b}} \mathrm{Ph}\right), 6.52$ / 5.99, 5.75, 5.25 (3-H' / 5-H', 2-H', 4-H'), 6.03 / 5.19, 5.43 (5-H / 4-H, 2-H), 3.58 / 1.51, 1.30, 1.03, 0.68 (13-H / 14-H, 14-H', 15-H, 16-H), 2.21 / 0.78, 0.75, 0.43 (9-H / 10-H, 11-H, 12-H), 1.44 / 2.04, 1.35, 1.33 (7-H, 8-H / 6-H, 7-H', 8-H'). NOEDiff (benzene-d $\left.{ }_{6}, 600 / 600 \mathrm{MHz}\right): \delta\left({ }^{1} \mathrm{H}\right)_{\text {irr. }} / \delta\left({ }^{1} \mathrm{H}\right)_{\text {resp. }}=7.97 / 7.44,6.52,5.75,5.43,3.58,2.04(\mathrm{o}-\mathrm{H}$ of ${ }^{\mathrm{a}} \mathrm{Ph} / \mathrm{m}-\mathrm{H}$ of $\left.{ }^{\mathrm{a}} \mathrm{Ph}, 3-\mathrm{H}^{\prime}, 2-\mathrm{H}^{\prime}, 2-\mathrm{H}, 14-\mathrm{H}, 6-\mathrm{H}\right), 6.96 / 7.23,6.03,5.99,5.25,5.19,3.58$ (o-H of ${ }^{\mathrm{b}} \mathrm{Ph} / \mathrm{m}-$ $\mathrm{H}$ of $\left.{ }^{\mathrm{b}} \mathrm{Ph}, 5-\mathrm{H}, 5-\mathrm{H}^{\prime}, 4-\mathrm{H}^{\prime}, 4-\mathrm{H}, 13-\mathrm{H}\right), 6.52$ / 7.97, 5.75, 5.25 (3-H' / o-H of $\left.{ }^{\mathrm{a}} \mathrm{Ph}, 2-\mathrm{H}^{\prime}, 4-\mathrm{H}^{\prime}\right), 6.03$ / 6.96, 5.19, 0.26 (5-H / o-H of $\left.{ }^{b} \mathrm{Ph}, 4-\mathrm{H}, \mathrm{Si}\left(\mathrm{CH}_{3}\right)_{2}\right), 5.75$ / 7.97, 6.52, 5.43, 0.40 (2-H' / o-H of ${ }^{\mathrm{a}} \mathrm{Ph}, 3-\mathrm{H}^{\prime}$, 2-H, $\left.\mathrm{Si}\left(\mathrm{CH}_{3}\right)_{2}\right), 5.43$ / 7.97, 5.75, 0.40 (2-H / o-H of $\left.{ }^{\mathrm{a}} \mathrm{Ph}, 2-\mathrm{H}^{\prime}, \mathrm{Si}\left(\mathrm{CH}_{3}\right)_{2}\right), 5.25$ / 6.96, 6.52, 5.99, 3.58 (4-H' / o-H of ' $\mathrm{Ph}, 3-\mathrm{H}^{\prime}$, 5-H', 13-H), 5.19 / 6.96, 6.03 (4-H / o-H of $\left.{ }^{\text {b }} \mathrm{Ph}, 5-\mathrm{H}\right), 3.58$ / 7.97, 6.96, 1.51, 1.30, 1.03, 0.68 (13-H / o-H of ${ }^{\mathrm{a}} \mathrm{Ph}, \mathrm{o}-\mathrm{H}$ of $\left.{ }^{\mathrm{b}} \mathrm{Ph}, 14-\mathrm{H}, 14-\mathrm{H} ', 15-\mathrm{H}, 16-\mathrm{H}\right), 0.40$ / 5.75, $5.43\left(\mathrm{Si}\left(\mathrm{CH}_{3}\right)_{2} /\right.$ 
2-H', 2-H), 0.26 / 6.03, $5.99\left(\mathrm{Si}\left(\mathrm{CH}_{3}\right)_{2} / 5-\mathrm{H}, 5-\mathrm{H}^{\prime}\right)$. GCOSY (benzene-d, $\left.600 / 600 \mathrm{MHz}\right): \delta\left({ }^{1} \mathrm{H}\right) / \delta$ $\left({ }^{1} \mathrm{H}\right)=7.97 / 7.44\left(\mathrm{o}-\mathrm{H}\right.$ of ${ }^{\mathrm{a}} \mathrm{Ph} / \mathrm{m}-\mathrm{H}$ of $\left.{ }^{\mathrm{a}} \mathrm{Ph}\right), 7.44 / 7.97,7.28\left(\mathrm{~m}-\mathrm{H}\right.$ of ${ }^{\mathrm{a}} \mathrm{Ph} / \mathrm{o}-\mathrm{H}$ of ${ }^{\mathrm{a}} \mathrm{Ph}, \mathrm{p}-\mathrm{H}$ of $\left.{ }^{\mathrm{a}} \mathrm{Ph}\right)$, $7.28 / 7.44\left(\mathrm{p}-\mathrm{H}\right.$ of ${ }^{\mathrm{a}} \mathrm{Ph} / \mathrm{m}-\mathrm{H}$ of $\left.{ }^{\mathrm{a}} \mathrm{Ph}\right), 7.23 / 7.07,6.96\left(\mathrm{~m}-\mathrm{H}\right.$ of ${ }^{\mathrm{b}} \mathrm{Ph} / \mathrm{p}-\mathrm{H}$ of ${ }^{\mathrm{b}} \mathrm{Ph}, \mathrm{o}-\mathrm{H}$ of $\left.{ }^{\mathrm{b}} \mathrm{Ph}\right), 7.07 /$ 7.23 (p-H of ${ }^{b} \mathrm{Ph} / \mathrm{m}-\mathrm{H}$ of $\left.{ }^{b} \mathrm{Ph}\right), 6.96 / 7.23$ (o-H of ${ }^{b} \mathrm{Ph} / \mathrm{m}-\mathrm{H}$ of $\left.{ }^{b} \mathrm{Ph}\right), 6.52$ / 5.75, 5.25 (3-H' / 2-H', 4H'), 6.03 / 5.43, 5.19 (5-H / 2-H, 4-H), 5.99 / 5.25 (5-H' / 4-H'), 5.75 / 6.52, 5.99 (2-H' / 3-H', 5-H'), 5.43 / 6.03, 5.19 (2-H / 5-H, 4-H), 5.25 / 6.52, 5.75 (4-H’ / 3-H', 2-H'), 5.19 / 6.03, 5.43 (4-H / 5-H, 2H), 3.58 / 1.51, 1.30 (13-H / 14-H, 14-H'), 2.21 / 0.78 (9-H / 10-H), 2.04 / 1.44, 1.35 (6-H / 7-H, 7-H'), 1.51 / 3.58, 1.30, 1.03 (14-H / 13-H, 14-H', 15-H), 1.44 / 2.04, 1.35, 1.33 (7-H / 6-H, 7-H', 8-H'), 1.35 / 2.04, 1.44 ( 7-H' / 6-H, 7-H), 1.30 / 3.58, 1.51, 1.03 (14-H’ / 13-H, 14-H, 15-H), 1.03 / 0.68 (15-H / 16-H), 0.78 / 2.21, 0.75 (10-H / 9-H, 11-H), 0.75 / 0.78, 0.48 (11-H / 10-H, 12-H), 0.68 / 1.03 (16-H / 15-H), $0.48 / 0.75(12-\mathrm{H} / 11-\mathrm{H})$. GHSQC (benzene-d, $150 / 600 \mathrm{MHz}): \delta\left({ }^{13} \mathrm{C}\right) / \delta\left({ }^{1} \mathrm{H}\right)=141.8 / 7.97$ (o-C of ${ }^{a} \mathrm{Ph} / \mathrm{o}-\mathrm{H}$ of $\left.{ }^{\mathrm{a}} \mathrm{Ph}\right), 129.8 / 7.23$ (m-C of ${ }^{b} \mathrm{Ph} / \mathrm{m}-\mathrm{H}$ of $\left.{ }^{b} \mathrm{Ph}\right), 127.4 / 7.07$ (p-C of ${ }^{b} \mathrm{Ph} / \mathrm{p}-\mathrm{H}$ of $\left.{ }^{b} \mathrm{Ph}\right)$, $126.7 / 7.44$ (m-C of ${ }^{a} \mathrm{Ph} / \mathrm{m}-\mathrm{H}$ of $\left.{ }^{\mathrm{a}} \mathrm{Ph}\right), 123.9 / 7.28$ (p-C of ${ }^{\mathrm{a}} \mathrm{Ph} / \mathrm{p}-\mathrm{H}$ of $\left.{ }^{\mathrm{a}} \mathrm{Ph}\right), 123.1 / 6.96\left(\mathrm{o}-\mathrm{C}\right.$ of ${ }^{b} \mathrm{Ph} /$ o-H of $\left.{ }^{b} \mathrm{Ph}\right), 121.9$ / 6.52 (C3' / 3-H'), 112.1 / 5.25 (C4' / 4-H'), 111.9 / 5.19 (C4 / 4-H), 108.1 / 6.03 (C5 / 5-H), 107.6 / 5.99 (C5' / 5-H'), 105.6 / 5.75 (C2' / 2-H'), 105.4 / 5.43 (C2 / 2-H), 48.6 / 3.58 (C13 / 13-H), 43.0 / 2.21 (C9 / 9-H), 33.6 / 2.04 (C6 / 6-H), 31.2 / 1.51, 1.30 (C14 / 14-H, 14-H'), 30.9 / 1.44, 1.35 (C7 / 7-H, 7-H'), 29.2 / 0.78 (C10 / 10-H), 20.7 / 1.44, 1.33 (C8 / 8-H, 8-H'), 20.5 / 1.03 (C15 / 15-H), 19.1 / 0.75 (C11 / 11-H), 13.5 / 0.68 (C16 / 16-H), 12.6 / 0.43 (C12 / 12-H), -4.4 / 0.40 $\left(\mathrm{Si}\left(\mathrm{CH}_{3}\right)_{2} / \mathrm{Si}\left(\mathrm{CH}_{3}\right)_{2}\right),-6.3 / 0.26\left(\mathrm{Si}\left(\mathrm{CH}_{3}\right)_{2} / \mathrm{Si}\left(\mathrm{CH}_{3}\right)_{2}\right)$. GHMBC (benzene-d, $\left.150 / 600 \mathrm{MHz}\right): \delta\left({ }^{13} \mathrm{C}\right) /$ $\delta\left({ }^{1} \mathrm{H}\right)=240.1 / 6.96,3.58\left(\mathrm{Zr}-\mathrm{C}-\mathrm{N} / \mathrm{o}-\mathrm{H}\right.$ of $\left.{ }^{\mathrm{b}} \mathrm{Ph}, 13-\mathrm{H}\right), 179.1 / 7.44$ (ipso-C of ${ }^{\mathrm{a}} \mathrm{Ph} / \mathrm{m}-\mathrm{H}$ of $\left.{ }^{\mathrm{a}} \mathrm{Ph}\right), 142.6$ / 7.23 (ipso-C of ${ }^{\mathrm{b}} \mathrm{Ph} / \mathrm{m}-\mathrm{H}$ of ${ }^{\mathrm{b}} \mathrm{Ph}$ ), 141.8 / 7.97, 7.28 (o-C of ${ }^{\mathrm{a}} \mathrm{Ph} / \mathrm{o}-, \mathrm{p}-\mathrm{H}$ of ${ }^{\mathrm{a}} \mathrm{Ph}$ ), 138.8 / 6.03, 5.43, 5.19, 2.04 (C3 / 5-H, 2-H, 4-H, 6-H), 129.3 / 7.23 (m-C of ${ }^{b} \mathrm{Ph} / \mathrm{m}-\mathrm{H}$ of $\left.{ }^{\mathrm{b}} \mathrm{Ph}\right), 127.4$ / 6.96 (p-C of ${ }^{\mathrm{b}} \mathrm{Ph} /$ o-H of $\left.{ }^{b} \mathrm{Ph}\right), 123.9 / 7.97$ (p-C of ${ }^{a} \mathrm{Ph} / \mathrm{o}-\mathrm{H}$ of $\left.{ }^{\mathrm{a}} \mathrm{Ph}\right), 123.1 / 7.07,6.96$ (o-C of ${ }^{b} \mathrm{Ph} / \mathrm{p}-\mathrm{H}$ of ${ }^{b} \mathrm{Ph}, \mathrm{o}-\mathrm{H}$ of $\left.{ }^{\mathrm{b}} \mathrm{Ph}\right), 112.1$ / 5.99, 5.75 (C4' / 5-H', 2-H'), 111.9 / 6.03, 5.43, 2.04 (C4 / 5-H, 2-H, 6-H), $108.1 / 5.43$ (C5 / 2-H), 107.6 / 5.75 (C5' / 2-H'), 105.4 / 6.03, 5.19 (C2 / 5-H, 4-H), 102.3 / 6.03, 5.19, 5.43, 0.40, $0.26\left(\mathrm{C} 1 / 5-\mathrm{H}, 4-\mathrm{H}, 2-\mathrm{H}, \mathrm{Si}\left(\mathrm{CH}_{3}\right)_{2}\right), 102.0$ / 5.99, 5.75, 0.40, $0.26\left(\mathrm{Cl}^{\prime}\right.$ / 5-H', 2-H', $\left.\mathrm{Si}\left(\mathrm{CH}_{3}\right)_{2}\right), 48.6$ / 
1.51, 1.30, 1.03 (C13 / 14-H, 14-H', 15-H), 31.2 / 3.58, 1.03, 0.68 (C14 / 13-H, 15-H ,16-H), 30.9 / 2.04 (C7 / 6-H, 6-H'), 29.2 / 2.21, 0.75, 0.48 (C10 / 9-H, 11-H ,12-H), 20.5 / 3.58, 1.51, 1.30, 0.68 (C15 / 13-H, 14-H, 14-H', 16-H), 19.1 / 2.21, 0.78, 0.48 (C11 / 9-H, 10-H, 12-H), 13.5 / 1.51, 1.30, $1.03\left(\mathrm{C} 16\right.$ / 14-H, 14-H', 15-H), -4.4 / $0.26\left(\mathrm{Si}\left(\mathrm{CH}_{3}\right)_{2} / \mathrm{Si}\left(\mathrm{CH}_{3}\right)_{2}\right),-6.3 / 0.40\left(\mathrm{Si}\left(\mathrm{CH}_{3}\right)_{2} / \mathrm{Si}\left(\mathrm{CH}_{3}\right)_{2}\right)$.

Addition of $\mathrm{PMe}_{3}$ to complex 12, preparation of 17. $\mathrm{mp} 227^{\circ} \mathrm{C}$ (decomp.). Anal. Calcd. for $\mathrm{C}_{42} \mathrm{H}_{38} \mathrm{BF}_{10} \mathrm{PSiZr}$ (893.8): C 56.44, $\mathrm{H} 4.29$; found C 56.10, H 4.41\%. IR (KBr): $\widetilde{v}=3055(\mathrm{~m}), 2910$

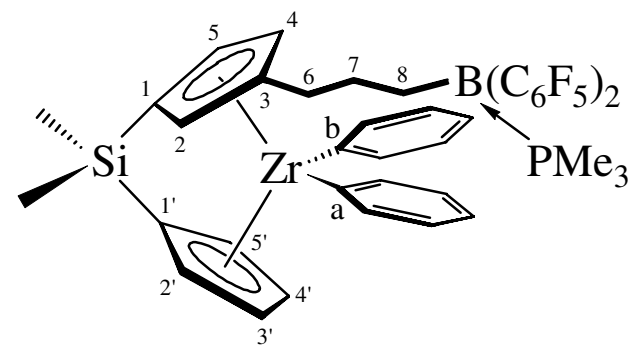
(m), 1637 (m), $1511(\mathrm{~s}), 1441$ (s), 1260 (s), 1155 (w), 1099 (s), $1043(\mathrm{~m}), 952(\mathrm{~s}), 805(\mathrm{~s}), 721(\mathrm{~s}), 666(\mathrm{~s}), 456(\mathrm{~m}) \mathrm{cm}^{-1}$. ${ }^{1} \mathrm{H}-\mathrm{NMR}$ (benzene-d $\left.\mathrm{d}_{6}, 600 \mathrm{MHz}\right): \delta=7.52,7.23,7.13$ (each m, 5H, o-, m-, p-H of ${ }^{\text {bh }}$ ), 7.31, 7.20, 7.08 (each m, 5H, o-, m-, p-H of $\left.{ }^{\mathrm{a}} \mathrm{Ph}\right), 6.52\left(\mathrm{~m}, 1 \mathrm{H}, 3-\mathrm{H}^{\prime}\right), 6.45\left(\mathrm{~m}, 1 \mathrm{H}, 4-\mathrm{H}^{\prime}\right), 6.45$ (m, 1H, 4-H), 5.63 (m, 1H, 5-H), 5.62 (m, 1H, 2-H'), 5.58 (m, 1H, 5-H'), 5.41 (m, 1H, 2-H), 2.01/1.89 (each m, each 1H, 6-H, 6-H'), 1.22/1.09 (each m, each 1H, 7-H, 7-H'), 0.74 (m, 2H, 8-H), 0.38 (d, ${ }^{2} \mathbf{J}_{\mathrm{PH}}=10.7 \mathrm{~Hz}, 9 \mathrm{H}, \mathrm{PMe}_{3}$ ), 0.27/0.26 (each s, each 3H, Si $\left.\left.\left(\mathrm{CH}_{3}\right)\right)_{2}\right) .{ }^{13} \mathrm{C}\left\{{ }^{1} \mathrm{H}\right\}-\mathrm{NMR}$ (benzene-d $\mathrm{d}_{6}, 150$ $\mathrm{MHz}): \delta \bullet=186.2,134.4,126.8,125.5$ (ipso-, o-, m-, p-C of ${ }^{\text {a }} \mathrm{Ph}$ ), 181.7, 136.2, 126.4, 125.9 (ipso-, o-, m-, p-C of $\left.{ }^{\text {b}} \mathrm{Ph}\right), 138.4$ (C3), 123.2 (C4'), 123.2 (C4), 121.5 (C3'), 114.7 (C2), 114.3 (C2'), 113.2 (C5), 112.1 (C5'), 100.4 (C1), 100.3 (C1'), 33.2 (C6), 29.4 (C7), 20.0 (C8), 8.9 (d, ${ }^{1} \mathrm{~J}_{\mathrm{CP}}=37.2 \mathrm{~Hz}, \mathrm{PMe}_{3}$ ), 5.5/-5.8 $\left(\mathrm{Si}\left(\mathrm{CH}_{3}\right)_{2}\right) ; 148.1\left({ }^{1} \mathrm{~J}_{\mathrm{CF}}=236.4 \mathrm{~Hz}\right.$, o-C of $\left.\mathrm{C}_{6} \mathrm{~F}_{5}\right), 139.4\left({ }^{1} \mathrm{~J}_{\mathrm{CF}}=251.3 \mathrm{~Hz}, \mathrm{p}-\mathrm{C}\right.$ of $\left.\mathrm{C}_{6} \mathrm{~F}_{5}\right), 137.4$ $\left({ }^{1} \mathrm{~J}_{\mathrm{CF}}=253.6 \mathrm{~Hz}, \mathrm{~m}-\mathrm{C}\right.$ of $\left.\mathrm{C}_{6} \mathrm{~F}_{5}\right), 119.7$ (ipso-C of $\mathrm{C}_{6} \mathrm{~F}_{5}$ ). 1D-TOCSY (benzene-d $\mathrm{d}_{6}, 600 / 600 \mathrm{MHz}$ ): $\delta$ $\left({ }^{1} \mathrm{H}\right)_{\text {irr. }} / \delta\left({ }^{1} \mathrm{H}\right)_{\text {resp. }}=7.52 / 7.23,7.13\left(\mathrm{o}-\mathrm{H}\right.$ of ${ }^{\mathrm{b}} \mathrm{Ph} / \mathrm{m}-, \mathrm{p}-\mathrm{H}$ of $\left.{ }^{\mathrm{b}} \mathrm{Ph}\right), 7.31 / 7.20,7.08\left(\mathrm{o}-\mathrm{H}\right.$ of ${ }^{\mathrm{a}} \mathrm{Ph} / \mathrm{m}-, \mathrm{p}-\mathrm{H}$ of $\left.{ }^{\mathrm{a}} \mathrm{Ph}\right), 6.52$ / 6.45, 5.62, 5.58 (3-H' / 4-H', 2-H', 5-H'), 5.41 / 6.45, 5.63 (2-H / 4-H, 5-H), 2.01 / 1.89, 1.22, 1.09, 0.74 (6-H / 6-H', 7-H', 7-H, 8-H). NOE-Diff (benzene-d $\left.{ }_{6}, 600 / 600 \mathrm{MHz}\right): \delta\left({ }^{1} \mathrm{H}\right)_{\text {irr. }} / \delta$ $\left({ }^{1} \mathrm{H}\right)_{\text {resp. }}=6.52 / 7.31,6.45,5.62\left(3-\mathrm{H}^{\prime} / \mathrm{o}-\mathrm{H}\right.$ of $\left.{ }^{\mathrm{a}} \mathrm{Ph}, 4-\mathrm{H}^{\prime}, 2-\mathrm{H}^{\prime}\right), 6.45$ / 7.52, 6.52, 5.63, 5.58 (4-H' / o-H of ${ }^{\mathrm{b}} \mathrm{Ph}, 3-\mathrm{H}^{\prime}$, 5-H, 5-H'), 5.63, 5.62 / 7.52, 7.31, 6.52, 6.45, 5.41, 0.27, 0.26 (5-H, 2-H' / o-H of ${ }^{\mathrm{b}} \mathrm{Ph}, \mathrm{o}-$ 
$\mathrm{H}$ of ${ }^{\mathrm{a}} \mathrm{Ph}, 3-\mathrm{H}$ ', 4-H, 5-H, $\left.\mathrm{Si}\left(\mathrm{CH}_{3}\right)_{2}, \mathrm{Si}\left(\mathrm{CH}_{3}\right)_{2}\right), 5.58$ / 7.52, 6.45, 0.26 (5-H' / o-H of ${ }^{\mathrm{b}} \mathrm{Ph}, 4-\mathrm{H}^{\prime}$, $\left.\mathrm{Si}\left(\mathrm{CH}_{3}\right)_{2}\right), 5.41 / 7.31,1.89$ (2-H / o-H of $\left.{ }^{\mathrm{a}} \mathrm{Ph}, 6-\mathrm{H}^{\prime}\right), 2.01 / 7.31,6.45$ (6-H / o-H of $\left.{ }^{\mathrm{a}} \mathrm{Ph}, 4-\mathrm{H}\right), 1.89$ / 7.31, 5.41 (6-H / o-H of $\left.{ }^{a} \mathrm{Ph}, 2-\mathrm{H}\right), 0.27$ / 5.62, $\left.5.41\left(\mathrm{Si}_{\left(\mathrm{CH}_{3}\right.}\right)_{2} / 2-\mathrm{H}^{\prime}, 2-\mathrm{H}\right), 0.26$ / 5.63, $5.58\left(\mathrm{Si}\left(\mathrm{CH}_{3}\right)_{2} /\right.$ 5-H, 5-H'). ${ }^{11} \mathrm{~B}\left\{{ }^{1} \mathrm{H}\right\}-\mathrm{NMR}$ (benzene-d, $\left.64 \mathrm{MHz}\right): \delta=-14.1\left(\mathrm{v}_{1 / 2}=258 \mathrm{~Hz}\right) .{ }^{31} \mathrm{P}\left\{{ }^{1} \mathrm{H}\right\}-\mathrm{NMR}$ (benzene-d ${ }_{6}$, $81 \mathrm{MHz}): \delta=-10.2\left(\mathrm{v}_{1 / 2}=115 \mathrm{~Hz}\right) .{ }^{19} \mathrm{~F}-\mathrm{NMR}\left(\right.$ benzene-d $\left._{6}, 564 \mathrm{MHz}\right): \delta=-130.7\left(\mathrm{~m}, 2 \mathrm{~F}, \mathrm{o}-\mathrm{F}\right.$ of $\left.\mathrm{C}_{6} \mathrm{~F}_{5}\right)$, $-130.8\left(\mathrm{~m}, 2 \mathrm{~F}, \mathrm{o}-\mathrm{F}\right.$ ' of $\left.\mathrm{C}_{6} \mathrm{~F}_{5}\right),-158.3\left(\mathrm{t},{ }^{3} \mathrm{~J}_{\mathrm{FF}}=20.8 \mathrm{~Hz}, 2 \mathrm{~F}, \mathrm{p}-\mathrm{F}\right.$ of $\left.\mathrm{C}_{6} \mathrm{~F}_{5}\right),-163.6\left(\mathrm{~m}, 4 \mathrm{~F}, \mathrm{~m}-\mathrm{F}\right.$ of $\left.\mathrm{C}_{6} \mathrm{~F}_{5}\right)$. GCOSY (benzene-d $\left.{ }_{6}, 600 / 600 \mathrm{MHz}\right): \delta\left({ }^{1} \mathrm{H}\right) / \delta\left({ }^{1} \mathrm{H}\right)=7.52 / 7.23\left(\mathrm{o}-\mathrm{H}\right.$ of ${ }^{\mathrm{b}} \mathrm{Ph} / \mathrm{m}-\mathrm{H}$ of $\left.{ }^{b} \mathrm{Ph}\right), 7.31 /$ 7.20 (o-H of ${ }^{a} \mathrm{Ph} / \mathrm{m}-\mathrm{H}$ of $\left.{ }^{\mathrm{a}} \mathrm{Ph}\right), 7.23 / 7.52,7.13\left(\mathrm{~m}-\mathrm{H}\right.$ of ${ }^{b} \mathrm{Ph} / \mathrm{o}-\mathrm{H}$ of ${ }^{b} \mathrm{Ph}, \mathrm{p}-\mathrm{H}$ of $\left.{ }^{b} \mathrm{Ph}\right), 7.20 / 7.31,7.08$ (m-H of ${ }^{a} \mathrm{Ph} / \mathrm{o}-\mathrm{H}$ of ${ }^{\mathrm{a}} \mathrm{Ph}, \mathrm{p}-\mathrm{H}$ of $\left.{ }^{\mathrm{a}} \mathrm{Ph}\right), 7.13 / 7.23\left(\mathrm{p}-\mathrm{H}\right.$ of ${ }^{\mathrm{b}} \mathrm{Ph} / \mathrm{m}-\mathrm{H}$ of $\left.{ }^{b} \mathrm{Ph}\right), 7.08 / 7.20\left(\mathrm{p}-\mathrm{H}\right.$ of ${ }^{\mathrm{a}} \mathrm{Ph} / \mathrm{m}-$ $\mathrm{H}$ of $\left.{ }^{\mathrm{a}} \mathrm{Ph}\right), 6.52$ / 6.45, 5.62 (3-H' / 4-H', 2-H'), 6.45 / 6.52, 5.63, 5.62, 5.58 (4-H, 4-H' / 3-H', 5-H, 2H', 5-H'), 5.63 / 6.45, 5.41 (5-H / 4-H, 2-H), 5.62 / 6.52, 5.58 (2-H' / 3-H', 5-H'), 5.41 / 6.45, 5.63 (2H / 4-H, 5-H), 2.01 / 1.89, 1.09 (6-H / 6-H', 7-H'), 1.89 / 2.01, 1.22 (6-H' / 6-H, 7-H), 1.22 / 2.01, 1.89, 1.09, 0.74 (7-H / 6-H, 6-H', 7-H', 8-H), 1.09 / 2.01, 1.89, 1.22, 0.74 (7-H' / 6-H, 6-H', 7-H, 8-H), $0.74 / 1.22(8-\mathrm{H} / 7-\mathrm{H})$. GHSQC (benzene-d, $150 / 600 \mathrm{MHz}): \delta\left({ }^{13} \mathrm{C}\right) / \delta\left({ }^{1} \mathrm{H}\right)=134.4 / 7.31(\mathrm{o}-\mathrm{C}$ of ${ }^{a} \mathrm{Ph} / \mathrm{o}-\mathrm{H}$ of $\left.{ }^{\mathrm{a}} \mathrm{Ph}\right), 126.8 / 7.20\left(\mathrm{~m}-\mathrm{C}\right.$ of ${ }^{\mathrm{a}} \mathrm{Ph} / \mathrm{m}-\mathrm{H}$ of $\left.{ }^{\mathrm{a}} \mathrm{Ph}\right), 125.5 / 7.08$ (p-C of ${ }^{\mathrm{a}} \mathrm{Ph} / \mathrm{p}-\mathrm{H}$ of $\left.{ }^{\mathrm{a}} \mathrm{Ph}\right), 136.2$ / $7.52\left(\mathrm{o}-\mathrm{C}\right.$ of ${ }^{b} \mathrm{Ph} / \mathrm{o}-\mathrm{H}$ of $\left.{ }^{b} \mathrm{Ph}\right), 126.4 / 7.23$ (m-C of ${ }^{b} \mathrm{Ph} / \mathrm{m}-\mathrm{H}$ of $\left.{ }^{b} \mathrm{Ph}\right), 125.9 / 7.13$ (p-C of ${ }^{b} \mathrm{Ph} / \mathrm{p}-\mathrm{H}$ of $\left.{ }^{\mathrm{b}} \mathrm{Ph}\right), 123.2$ / 6.45 (C4' / 4-H'), 123.2 / 6.45 (C4 / 4-H), 121.5 / 6.52 (C3' / 3-H'), 114.7 / 5.41 (C2 / 2 H), 114.3 / 5.62 (C2' / 2-H'), 113.2 / 5.63 (C5 / 5-H), 112.1 / 5.58 (C5' / 5-H'), 33.2 / 2.01, 1.89 (C6 / 6-H, 6-H'), 29.4 / 1.22, 1.09 (C7 / 7-H, 7-H'), 20.0 / 0.74 (C8 / 8-H), 8.9 / $0.38\left(\mathrm{PMe}_{3} / \mathrm{PMe}_{3}\right),-5.5$ / $0.26\left(\mathrm{Si}\left(\mathrm{CH}_{3}\right)_{2} / \mathrm{Si}\left(\mathrm{CH}_{3}\right)_{2}\right),-5.8 / 0.27\left(\mathrm{Si}\left(\mathrm{CH}_{3}\right)_{2} / \mathrm{Si}\left(\mathrm{CH}_{3}\right)_{2}\right) . \mathrm{GHMBC}($ benzene-d, $150 / 600 \mathrm{MHz}): \delta$ $\left({ }^{13} \mathrm{C}\right) / \delta\left({ }^{1} \mathrm{H}\right)=186.2 / 7.31,7.20\left(\right.$ ipso-C of ${ }^{\mathrm{a}} \mathrm{Ph} / \mathrm{o}-, \mathrm{m}-\mathrm{H}$ of $\left.{ }^{\mathrm{a}} \mathrm{Ph}\right), 181.7 / 7.52,7.23\left(\right.$ ipso-C of ${ }^{\mathrm{b}} \mathrm{Ph} / \mathrm{o}-$, m-H of $\left.{ }^{b} \mathrm{Ph}\right), 138.4$ / 6.45, 5.63, 5.41, 2.01, 1.89, 1.22, 1.09 (C3 / 4-H, 5-H, 2-H, 6-H, 6-H', 7-H, 7-H'), 136.2 / 7.52, 7.13 (o-C of ${ }^{b} \mathrm{Ph} / \mathrm{o}-, \mathrm{p}-\mathrm{H}$ of $\left.{ }^{\mathrm{b}} \mathrm{Ph}\right), 134.4 / 7.31,7.08$ (o-C of ${ }^{\mathrm{a}} \mathrm{Ph} / \mathrm{o}-, \mathrm{p}-\mathrm{H}$ of $\left.{ }^{\mathrm{a}} \mathrm{Ph}\right), 125.9$ / $7.52\left(\mathrm{p}-\mathrm{C}\right.$ of ${ }^{b} \mathrm{Ph} / \mathrm{o}-\mathrm{H}$ of $\left.{ }^{b} \mathrm{Ph}\right), 125.5 / 7.31\left(\mathrm{p}-\mathrm{C}\right.$ of ${ }^{\mathrm{a}} \mathrm{Ph} / \mathrm{o}-\mathrm{H}$ of $\left.{ }^{\mathrm{a}} \mathrm{Ph}\right), 123.2$ / 6.52, 5.58 (C4' / 3-H', 5H'), 123.2 / 5.63, 5.41, 2.01, 1.89 (C4 / 5-H, 2-H, 6-H, 6-H'), 121.5 / 6.45, 5.62, 5.58 (C3' / 4-H', 2- 
H', 5-H'), 114.7 / 6.45, 5.63, 2.01, 1.89 (C2 / 4-H, 5-H, 6-H, 6-H'), 114.3 / 5.58 (C2' / 5-H'), 113.2 / 6.45, 5.41 (C5 / 4-H, 2-H), 112.1 / 6.52, 5.62 (C5' / 3-H', 2-H'), 100.4 / 6.45, 5.63, 5.41, 0.27, 0.26

(C1 / 4-H, 5-H, 2-H, Si( $\left.\left(\mathrm{CH}_{3}\right)_{2}, \mathrm{Si}\left(\mathrm{CH}_{3}\right)_{2}\right), 100.3$ / 5.58, 5.62, 0.27, 0.26 (C1' / 5-H', 2-H', $\mathrm{Si}\left(\mathrm{CH}_{3}\right)_{2}$, $\left.\mathrm{Si}\left(\mathrm{CH}_{3}\right)_{2}\right), 33.2$ / 1.22, 1.09, 0.74 (C6 / 7-H, 7-H', 8-H), 20.0 / 2.01, 1.89, 1.22, 1.09 (C8 / 6-H, 6-H', 7H, 7-H'), 29.4 / 2.01, 1.89, $0.74\left(\mathrm{C} 7 / 6-\mathrm{H}, 6-\mathrm{H}^{\prime}, 8-\mathrm{H}\right), 8.9$ / $0.38\left(\mathrm{PMe}_{3} / \mathrm{PMe}_{3}\right),-5.5 / 0.27\left(\mathrm{Si}_{\left(\mathrm{CH}_{3}\right.}\right)_{2} /$ $\left.\mathrm{Si}\left(\mathrm{CH}_{3}\right)_{2}\right),-5.8 / 0.26\left(\mathrm{Si}\left(\mathrm{CH}_{3}\right)_{2} / \mathrm{Si}\left(\mathrm{CH}_{3}\right)_{2}\right)$.

\section{Thermolysis of complex 12 in the presence of excess $\mathrm{PMe}_{3}$, preparation of the} (aryne)zirconocene complex 18. $\mathrm{mp} 224^{\circ} \mathrm{C}$ (decomp.). Anal. Calcd. for $\mathrm{C}_{39} \mathrm{H}_{41} \mathrm{BF}_{10} \mathrm{P}$. $\mathrm{SiZr}$ (891.8): $\mathrm{C}$ 52.53, H 4.63; found: C 52.53, H 3.69\%. IR (KBr): $\widetilde{v}=2917$ (m), $1644(\mathrm{~m}), 1511(\mathrm{~s}), 1441(\mathrm{~s}), 1273$ (m), $1246(\mathrm{~m}), 1148(\mathrm{w}), 1085(\mathrm{~m}), 1029(\mathrm{w}), 959(\mathrm{~m}), 805(\mathrm{~s}), 728(\mathrm{~s}), 672(\mathrm{~s}), 553(\mathrm{w}), 449(\mathrm{w}) \mathrm{cm}^{-1}$.

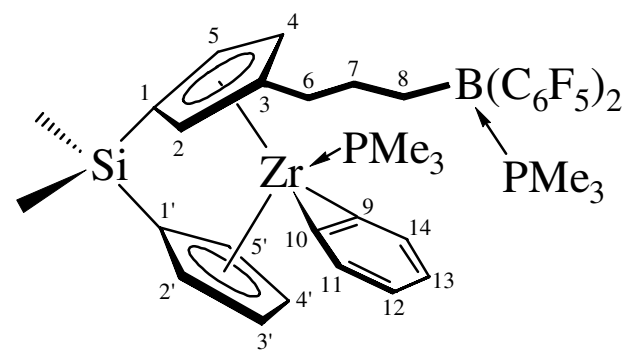

${ }^{1} \mathrm{H}$ NMR (benzene-d $\left.{ }_{6}, 600 \mathrm{MHz}\right): \delta=8.09(\mathrm{~m}, 1 \mathrm{H}, 11-\mathrm{H}), 7.66$ (m, 1H, 14-H), $7.33(\mathrm{~m}, 2 \mathrm{H}, 12-\mathrm{H}, 13-\mathrm{H}), 6.28\left(\mathrm{~m}, 1 \mathrm{H}, 2-\mathrm{H}^{\prime}\right)$, $6.02(\mathrm{~m}, 1 \mathrm{H}, 2-\mathrm{H}), 5.26\left(\mathrm{~m}, 2 \mathrm{H}, 4-\mathrm{H}, 5-\mathrm{H}^{\prime}\right), 5.25(\mathrm{~m}, 1 \mathrm{H}, 4-$ H'), $5.19(\mathrm{~m}, 1 \mathrm{H}, 5-\mathrm{H}), 5.16\left(\mathrm{~m}, 1 \mathrm{H}, 3-\mathrm{H}^{\prime}\right), 1.53 / 1.29$ (each m, each 1H, 6-H, 6-H'), $1.08\left(\mathrm{~d},{ }^{2} \mathrm{~J}_{\mathrm{PH}}=6.1 \mathrm{~Hz}, 9 \mathrm{H}, \mathrm{Zr}-\mathrm{PMe}_{3}\right)$, 1.07/0.74 (each m, each 1H, 7-H, 7-H'), $0.54(\mathrm{~m}, 2 \mathrm{H}, 8-\mathrm{H}), 0.38\left(\mathrm{~d},{ }^{2} \mathrm{~J}_{\mathrm{PH}}=11.4 \mathrm{~Hz}, 9 \mathrm{H}, \mathrm{B}-\mathrm{PMe}_{3}\right)$, 0.48/0.37 (each s, each 3H, $\left.\mathrm{Si}\left(\mathrm{CH}_{3}\right)_{2}\right) .{ }^{13} \mathrm{C}\left\{{ }^{1} \mathrm{H}\right\}$ NMR (benzene-d ${ }_{6}, 150 \mathrm{MHz}$ ): $\delta=178.5(\mathrm{C} 10), 157.8$ (C9), 134.1 (C14), 132.3 (C3), 131.6 (C11), 127.4 (C12, C13), 114.2 (C3'), 109.6 (C4), 108.5 (C4'), 106.9 (C2), 106.2 (C2'), 101.5 (C1'), 100.5 (C5'), 100.2 (C1), 99.8 (C5), 33.0 (C6), 28.0 (C7), 20.4 (C8), $\left.18.2\left(\mathrm{~d},{ }^{1} \mathrm{~J}_{\mathrm{CP}}=17.4 \mathrm{~Hz}, \mathrm{Zr}-\mathrm{PMe}_{3}\right), 8.8\left(\mathrm{~d},{ }^{1} \mathrm{~J}_{\mathrm{CP}}=36.6 \mathrm{~Hz}, \mathrm{~B}-\mathrm{PMe}_{3}\right),-4.8 /-5.1\left(\mathrm{Si}_{\left(\mathrm{CH}_{3}\right.}\right)_{2}\right) ; 148.1$ $\left({ }^{1} \mathrm{~J}_{\mathrm{CF}}=241.3 \mathrm{~Hz}, \mathrm{o}-\mathrm{C}\right.$ of $\left.\mathrm{C}_{6} \mathrm{~F}_{5}\right), 139.4\left({ }^{1} \mathrm{~J}_{\mathrm{CF}}=252.4 \mathrm{~Hz}, \mathrm{p}-\mathrm{C}\right.$ of $\left.\mathrm{C}_{6} \mathrm{~F}_{5}\right), 137.5\left({ }^{1} \mathrm{~J}_{\mathrm{CF}}=257.8 \mathrm{~Hz}, \mathrm{~m}-\mathrm{C}\right.$ of $\left.\mathrm{C}_{6} \mathrm{~F}_{5}\right), 119.9$ (ipso-C of $\left.\mathrm{C}_{6} \mathrm{~F}_{5}\right) .{ }^{11} \mathrm{~B}\left\{{ }^{1} \mathrm{H}\right\}$ NMR (benzene- $\left.\mathrm{d}_{6}, 64 \mathrm{MHz}\right): \delta=-14.0\left(v_{1 / 2}=258 \mathrm{~Hz}\right) .{ }^{31} \mathrm{P}\left\{{ }^{1} \mathrm{H}\right\}$ 
NMR (benzene-d $\left.\mathrm{d}_{6}, 81 \mathrm{MHz}\right): \delta=-7.9\left(\mathrm{Zr}-\mathrm{PMe}_{3}, \mathrm{v}_{1 / 2}=7 \mathrm{~Hz}\right),-10.0\left(\mathrm{~B}_{-} \mathrm{PMe}_{3}, \mathrm{v}_{1 / 2}=120 \mathrm{~Hz}\right) .{ }^{19} \mathrm{~F} \mathrm{NMR}$ (benzene-d $\left.{ }_{6}, 564 \mathrm{MHz}\right): \delta=-129.9\left(\mathrm{~m}, 2 \mathrm{~F}, \mathrm{o}-\mathrm{F}\right.$ of $\left.\mathrm{C}_{6} \mathrm{~F}_{5}\right),-130.3\left(\mathrm{~m}, 2 \mathrm{~F}, \mathrm{o}-\mathrm{F}^{\prime}\right.$ of $\left.\mathrm{C}_{6} \mathrm{~F}_{5}\right),-157.9\left(\mathrm{t},{ }^{3} \mathrm{~J}_{\mathrm{FF}}=\right.$ $20.7 \mathrm{~Hz}, 1 \mathrm{~F}, \mathrm{p}-\mathrm{F}^{\prime}$ of $\left.\mathrm{C}_{6} \mathrm{~F}_{5}\right),-158.4\left(\mathrm{t},{ }^{3} \mathrm{~J}_{\mathrm{FF}}=20.8 \mathrm{~Hz}, 1 \mathrm{~F}, \mathrm{p}-\mathrm{F}\right.$ of $\left.\mathrm{C}_{6} \mathrm{~F}_{5}\right),-163.1\left(\mathrm{~m}, 2 \mathrm{~F}, \mathrm{~m}-\mathrm{F}^{\prime}\right.$ of $\left.\mathrm{C}_{6} \mathrm{~F}_{5}\right),-$ $163.2\left(\mathrm{~m}, 2 \mathrm{~F}, \mathrm{~m}-\mathrm{F}\right.$ of $\left.\mathrm{C}_{6} \mathrm{~F}_{5}\right) .1 \mathrm{D}-\mathrm{TOCSY}\left(\right.$ benzene-d $\left._{6}, 600 / 600 \mathrm{MHz}\right): \delta\left({ }^{1} \mathrm{H}\right)_{\text {irr. }} / \delta\left({ }^{1} \mathrm{H}\right)_{\text {resp. }}=8.09 / 7.66$ 7.33 (11-H / 14-H, 12-H, 13-H), 6.28 / 5.26, 5.25, 5.16 (2-H' / 5-H', 4-H', 3-H'), 6.02 / 5.26, 5.19 (2-H / 4-H, 5-H), 1.53 / 1.29, 1.07, 0.74, 0.54 (6-H / 6-H', 7-H', 7-H, 8-H). NOE-Diff (benzene-d, 600 / $600 \mathrm{MHz}): \delta\left({ }^{1} \mathrm{H}\right)_{\text {irr. }} / \delta\left({ }^{1} \mathrm{H}\right)_{\text {resp. }}=8.09$ / 7.33, 6.28, 6.02, $5.16\left(11-\mathrm{H} / 12-\mathrm{H}, 13-\mathrm{H}, 2-\mathrm{H}^{\prime}, 2-\mathrm{H}, 3-\mathrm{H}^{\prime}\right), 7.66$ / 7.33, 1.08 (14-H / 12-H, 13-H, Zr-PMe $), 6.28$ / 6.02, 5.16, 0.48 (2-H' / 2-H, 3-H', Si $\left.\left(\mathrm{CH}_{3}\right)_{2}\right), 6.02$ / 8.09, 6.28, 0.48 (2-H / 11-H, 2-H', Si $\left.\left(\mathrm{CH}_{3}\right)_{2}\right)$, 5.19 / 8.09, 1.08, 0.38 (5-H / 11-H, Zr-PMe, $\left.\mathrm{Si}\left(\mathrm{CH}_{3}\right)_{2}\right)$, 5.16 / 6.28, 5.25 (3-H' / 2-H', 4-H'), 1.08 / 7.66, 5.26, 5.25, 5.19 (Zr-PMe / 14-H, 5-H', 4-H, 4-H', 5H), 0.48 / 6.28, $6.02\left(\mathrm{Si}\left(\mathrm{CH}_{3}\right)_{2} / 2-\mathrm{H}^{\prime}, 2-\mathrm{H}\right), 0.37$ / 5.26, $5.19\left(\mathrm{Si}\left(\mathrm{CH}_{3}\right)_{2} /\right.$ 5-H', 5-H). GCOSY (benzene$\left.\mathrm{d}_{6}, 600 / 600 \mathrm{MHz}\right): \delta\left({ }^{1} \mathrm{H}\right) / \delta\left({ }^{1} \mathrm{H}\right)=8.09$ / $7.33(11-\mathrm{H} / 12-\mathrm{H}, 13-\mathrm{H}), 7.66 / 7.33$ (14-H / 12-H, 13-H), 7.33 / 8.09, 7.66 (12-H, 13-H / 11-H, 14-H), 6.28 / 5.26, 5.16 (2-H' / 5-H', 3-H'), 6.02 / 5.26, 5.19 (2H / 4-H, 5-H), 5.26 / 6.02, 5.25, 5.19 (4-H , 5-H' / 2-H, 4-H', 5-H), 5.25 / 5.26, 5.16 (4-H' / 5-H', 3H'), 5.19 / 6.02 (5-H / 2-H), 5.16 / 6.28, 5.25 (3-H' / 2-H', 4-H'), 1.53 / 1.29, 1.07, 0.74 (6-H / 6-H', 7H, 7-H'), 1.29 / 1.53, 1.07, 0.74 (6-H' / 6-H, 7-H, 7-H'), 1.07 / 1.53, 1.29, 0.74 (7-H / 6-H, 6-H', 7H'), 0.74 / 1.53, 1.29, 1.07, 0.54 (7-H’ / 6-H, 6-H', 7-H, 8-H), 0.54 / 0.74 (8-H / 7-H'). GHSQC (benzene-d $\left.{ }_{6}, 150 / 600 \mathrm{MHz}\right): \delta\left({ }^{13} \mathrm{C}\right) / \delta\left({ }^{1} \mathrm{H}\right)=134.1 / 7.66(\mathrm{C} 14 / 14-\mathrm{H}), 131.6 / 8.09(\mathrm{C} 11 / 11-\mathrm{H})$, 127.4 / 7.33 (C12, C13 / 12-H, 13-H), 114.2 / 5.16 (C3’ / 3-H'), 109.6 / 5.26 (C4 / 4-H), 108.5 / 5.25 (C4' / 4-H'), 106.9 / 6.02 (C2 / 2-H), 106.2 / 6.28 (C2' / 2-H'), 100.5 / 5.26 (C5' / 5-H'), 99.8 / 5.19 (C5 / 5-H), 33.0 / 1.53, 1.29 (C6 / 6-H, 6-H'), 28.0 / 1.07, 0.74 (C7 / 7-H, 7-H'), 20.4 / 0.54 (C8 / 8-H),

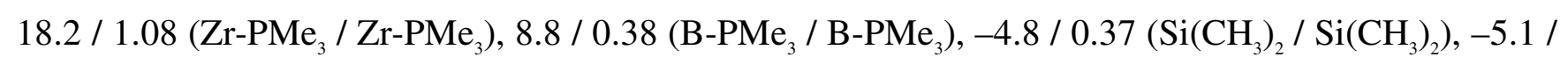
$0.48\left(\mathrm{Si}\left(\mathrm{CH}_{3}\right)_{2} / \mathrm{Si}\left(\mathrm{CH}_{3}\right)_{2}\right)$. GHMBC (benzene-d $\left.{ }_{6}, 150 / 600 \mathrm{MHz}\right): \delta\left({ }^{13} \mathrm{C}\right) / \delta\left({ }^{1} \mathrm{H}\right)=178.5 / 8.09,7.66$ 7.33 (C10 / 11-H, 14-H, 12-H, 13-H), 157.8 / 8.09, 7.66, 7.33 (C9 / 11-H, 14-H, 12-H, 13-H), $134.1 /$ 8.09, 7.33 (C14 / 11-H, 12-H, 13-H), 132.3 / 6.02, 5.26, 5.19, 1.53, 1.29 (C3 / 2-H, 4-H, 5-H, 6-H, 6- 
H'), 131.6 / 7.66, 7.33 (C11 / 14-H, 12-H, 13-H), 127.4 / 8.09, 7.66, 7.33 (C12, C13 / 11-H, 14-H, 12-

H, 13-H), 114.2 / 6.28, 5.26 (C3' / 2-H', 5-H'), 109.6 / 6.02, 5.19, 1.53, 1.29 (C4 / 2-H, 5-H, 6-H, 6H'), 108.5 / 6.28, 5.26, 5.16 (C4' / 2-H', 5-H', 3-H'), 106.9 / 5.26, 5.19, 1.53, 1.29 (C2 / 4-H, 5-H, 6H, 6-H'), 106.2 / 5.26, 5.16 (C2' / 5-H', 3-H'), 101.5 / 6.28, 5.26, 0.48, 0.37 (C1' / 2-H', 5-H', $\left.\mathrm{Si}\left(\mathrm{CH}_{3}\right)_{2}, \mathrm{Si}\left(\mathrm{CH}_{3}\right)_{2}\right), 100.5$ / 6.28, 5.16 (C5' / 2-H', 3-H'), 100.2 / 6.02, 5.26, 5.19, 0.48, 0.37 (C1 / 2-H, 4-H, 5-H, $\left.\mathrm{Si}\left(\mathrm{CH}_{3}\right)_{2}, \mathrm{Si}\left(\mathrm{CH}_{3}\right)_{2}\right), 99.8$ / 6.02, 5.26 (C5 / 2-H, 4-H), 33.0 / 0.74, 0.54 (C6 / 7-H', 8-H), 28.0 / 1.53, 1.29, 0.74, 0.54 (C7 / 6-H, 6-H', 7-H’, 8-H), 20.4 / 1.53, 1.29, (C8 / 6-H, 6-H'), 18.2 / 1.08 (Zr-

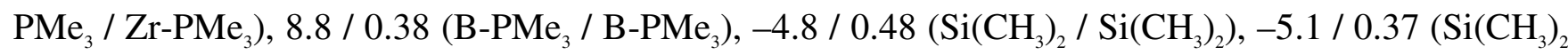
/ $\left.\left.\mathrm{Si}\left(\mathrm{CH}_{3}\right)\right)_{2}\right) .{ }^{19} \mathrm{~F} /{ }^{19} \mathrm{~F}$ GCOSY (benzene-d 6 , $\left.564 / 564 \mathrm{MHz}\right): \delta\left({ }^{19} \mathrm{~F}\right) / \delta\left({ }^{19} \mathrm{~F}\right)=-129.9 /-163.2(\mathrm{o}-\mathrm{F} / \mathrm{m}-\mathrm{F})$, -130.3 / -163.1 (o-F' / m-F'), -158.4 / -163.2 (p-F/ m-F), -157.9 / -163.1 (p-F' / m-F'), -163.1 / 157.9, -130.3 (m-F/ p-F, o-F), -163.2 / -158.4, -129.9 (m-F / p-F, o-F).

Reaction of complex 12 with $\mathrm{N}$-methylbenzimidazole, preparation of 19a. $\mathrm{mp} 181^{\circ} \mathrm{C}$ (decomp.).

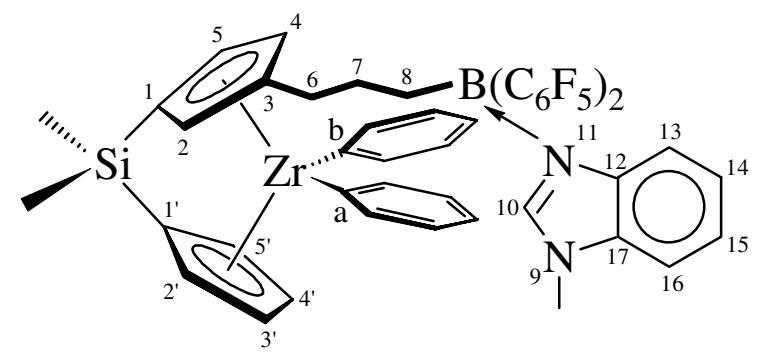
Anal. Calcd. for $\mathrm{C}_{47} \mathrm{H}_{37} \mathrm{~N}_{2} \mathrm{BF}_{10} \mathrm{SiZr}$ (949.9): C 59.43, $\mathrm{H}$ 3.93, N 2.95; found: C 59.34, H 4.19, N 3.11\%. IR $(\mathrm{KBr}): \widetilde{v}=3028(\mathrm{w}), 2930(\mathrm{w}), 1637(\mathrm{~s}), 1546(\mathrm{~m})$, $1504(\mathrm{~s}), 1456(\mathrm{~s}), 1351(\mathrm{w}), 1239(\mathrm{~s}), 1204(\mathrm{w}), 1085$ (s), $966(\mathrm{~s}), 805(\mathrm{~s}), 742(\mathrm{~s}), 721(\mathrm{~s}), 694(\mathrm{~s}), 456(\mathrm{~m})$ $\mathrm{cm}^{-1} .{ }^{1} \mathrm{H}-\mathrm{NMR}$ (benzene-d, $600 \mathrm{MHz}$ ): $\delta=7.72(\mathrm{~m}, 1 \mathrm{H}, 13-\mathrm{H}$ ), 7.56, 7.26, 7.15 (each m, 5H, o-, m-, p$\mathrm{H}$ of $\left.{ }^{b} \mathrm{Ph}\right), 7.36$ (s, 3H, 10-H), 7.35, 7.26, 7.12 (each m, 5H, o-, m-, p-H of $\left.{ }^{a} \mathrm{Ph}\right), 6.85(\mathrm{~m}, 1 \mathrm{H}, 14-\mathrm{H})$, $6.83(\mathrm{~m}, 1 \mathrm{H}, 15-\mathrm{H}), 6.58\left(\mathrm{~m}, 1 \mathrm{H}, 3-\mathrm{H}^{\prime}\right), 6.52(\mathrm{~m}, 1 \mathrm{H}, 4-\mathrm{H}), 6.47$ (m, 1H, 4-H'), $6.43(\mathrm{~m}, 1 \mathrm{H}, 16-\mathrm{H})$, $5.66(\mathrm{~m}, 1 \mathrm{H}, 5-\mathrm{H}), 5.64\left(\mathrm{~m}, 1 \mathrm{H}, 2-\mathrm{H}^{\prime}\right), 5.60\left(\mathrm{~m}, 1 \mathrm{H}, 5-\mathrm{H}^{\prime}\right), 5.48(\mathrm{~m}, 1 \mathrm{H}, 2-\mathrm{H}), 2.21(\mathrm{~s}, 3 \mathrm{H}, 9-\mathrm{H})$, 2.12/1.94 (each m, each 1H, 6-H, 6-H'), 1.49 (m, 2H, 8-H), 1.23/1.19 (each m, each 1H, 7-H, 7-H'), 0.28/0.25 (each s, each $\left.3 \mathrm{H}, \mathrm{Si}\left(\mathrm{CH}_{3}\right)_{2}\right) .{ }^{13} \mathrm{C}\left\{{ }^{1} \mathrm{H}\right\}-\mathrm{NMR}$ (benzene-d ${ }_{6}, 150 \mathrm{MHz}$ ): $\delta=186.1,134.4,126.5$, 125.7 (ipso-, o-, m-, p-C of ${ }^{a} \mathrm{Ph}$ ), 181.7, 136.3, 126.5, 126.1 (ipso-, o-, m-, p-C of ${ }^{b} \mathrm{Ph}$ ), 141.2 (C10), 138.9 (C3), 136.3 (C12), 132.7 (C17), 125.4 (C14), 125.1 (C15), 123.5 (C4'), 123.1 (C4), 121.8 (C3'), 
116.7 (C13), 114.9 (C2), 114.3 (C2'), 113.5 (C5), 112.4 (C5'), 110.7 (C16), 100.5 (C1), 100.1 (C1'), $33.4(\mathrm{C} 6), 30.6$ (C9), $\left.28.5(\mathrm{C} 7), 21.7(\mathrm{C} 8),-5.8 /-6.2\left(\mathrm{Si}_{\left(\mathrm{CH}_{3}\right)}\right)_{2}\right), 148.6\left({ }^{1} \mathrm{~J}_{\mathrm{CF}}=241.7 \mathrm{~Hz}, \mathrm{o}-\mathrm{C}\right.$ of $\left.\mathrm{C}_{6} \mathrm{~F}_{5}\right)$, $139.6\left({ }^{1} \mathrm{~J}_{\mathrm{CF}}=256.8 \mathrm{~Hz}, \mathrm{p}-\mathrm{C}\right.$ of $\left.\mathrm{C}_{6} \mathrm{~F}_{5}\right), 137.5\left({ }^{1} \mathrm{~J}_{\mathrm{CF}}=253.0 \mathrm{~Hz}, \mathrm{~m}-\mathrm{C}\right.$ of $\left.\mathrm{C}_{6} \mathrm{~F}_{5}\right) .{ }^{11} \mathrm{~B}\left\{{ }^{1} \mathrm{H}\right\}-\mathrm{NMR}$ (benzene- $\mathrm{d}_{6}$, $64 \mathrm{MHz}): \delta=-4.54\left(\mathrm{v}_{1 / 2}=383 \mathrm{~Hz}\right) .{ }^{19} \mathrm{~F}-\mathrm{NMR}$ (benzene- $\left.\mathrm{d}_{6}, 564 \mathrm{MHz}\right): \delta=-132.7\left(\mathrm{~m}, 4 \mathrm{~F}, \mathrm{o}-\mathrm{F}_{\text {of }} \mathrm{C}_{6} \mathrm{~F}_{5}\right)$, $-158.5\left(\mathrm{t},{ }^{3} \mathrm{~J}_{\mathrm{FF}}=21.1 \mathrm{~Hz}, 1 \mathrm{~F}, \mathrm{p}-\mathrm{F}\right.$ of $\left.\mathrm{C}_{6} \mathrm{~F}_{5}\right),-158.6\left(\mathrm{t},{ }^{3} \mathrm{~J}_{\mathrm{FF}}=21.1 \mathrm{~Hz}, 1 \mathrm{~F}, \mathrm{p}-\mathrm{F}{ }^{\prime}\right.$ of $\left.\mathrm{C}_{6} \mathrm{~F}_{5}\right),-164.2(\mathrm{~m}, 4 \mathrm{~F}, \mathrm{~m}-$ F of $\left.\mathrm{C}_{6} \mathrm{~F}_{5}\right) .1 \mathrm{D}-\mathrm{TOCSY}\left(\right.$ benzene-d $\left.\mathrm{d}_{6}, 600 / 600 \mathrm{MHz}\right): \delta\left({ }^{1} \mathrm{H}\right)_{\text {irr. }} / \delta\left({ }^{1} \mathrm{H}\right)_{\text {resp. }}=7.72 / 6.85,6.83,6.43(13-\mathrm{H}$ / 14-H, 15-H, 16-H), 7.56 / 7.26, 7.15 (o-H of ${ }^{b} \mathrm{Ph} / \mathrm{m}-$, p-H of $\left.{ }^{\mathrm{b}} \mathrm{Ph}\right), 7.35$ / 7.26, 7.12 (o-H of ${ }^{\mathrm{a}} \mathrm{Ph} / \mathrm{m}-$, p-H of $\left.{ }^{\mathrm{a}} \mathrm{Ph}\right), 6.58$ / 6.47, 5.64, 5.60 (3-H' / 4-H', 2-H', 5-H'), 5.48 / 6.52, 5.66 (2-H / 4-H, 5-H), 1.49 / 2.12, 1.94, 1.23, 1.19 (8-H / 6-H, 6-H', 7-H, 7-H'). NOE-Diff (benzene-d $\left.{ }_{6}, 600 / 600 \mathrm{MHz}\right): \delta\left({ }^{1} \mathrm{H}\right)_{\text {ir. }} / \delta$ $\left({ }^{1} \mathrm{H}\right)_{\text {resp. }}=7.72 / 6.85(13-\mathrm{H} / 14-\mathrm{H}), 7.56 / 7.35,7.26,6.52,6.47,5.66,5.60\left(\mathrm{o}-\mathrm{H}\right.$ of ${ }^{b} \mathrm{Ph} / \mathrm{o}-\mathrm{H}$ of ${ }^{a} \mathrm{Ph}$, m-H of ' $\left.\mathrm{Ph}, 4-\mathrm{H}, 4-\mathrm{H}^{\prime}, 5-\mathrm{H}, 5-\mathrm{H}^{\prime}\right), 7.36$ / 2.21 (10-H / 9-H), 7.35 / 7.56, 7.26, 6.58, 5.64, 5.48 (o-H of ${ }^{a} \mathrm{Ph} / \mathrm{o}-\mathrm{H}$ of ${ }^{b} \mathrm{Ph}, \mathrm{m}-\mathrm{H}$ of $\left.{ }^{a} \mathrm{Ph}, 4-\mathrm{H}^{\prime}, 2-\mathrm{H}^{\prime}, 2-\mathrm{H}\right), 6.58$ / 7.35, 6.47, 5.64 (3-H' / o-H of $\left.{ }^{\mathrm{a}} \mathrm{Ph}, 4-\mathrm{H}^{\prime}, 2-\mathrm{H}^{\prime}\right)$, 6.52 / 7.56, 5.66, 2.12 (4-H / o-H of $\left.{ }^{b} \mathrm{Ph}, 5-\mathrm{H}, 6-\mathrm{H}\right), 6.47$ / 7.56, 6.58, 5.60 (4-H' / o-H of ${ }^{b} \mathrm{Ph}, 3-\mathrm{H}^{\prime}, 5-$ H'), 6.43 / 6.83, 2.21 (16-H / 15-H, 9-H), 5.66 / 7.56, 6.52, 6.47, 0.25 (5-H / o-H of ${ }^{b} \mathrm{Ph}, 4-\mathrm{H}, 4-\mathrm{H}^{\prime}$, $\left.\mathrm{Si}\left(\mathrm{CH}_{3}\right)_{2}\right), 5.64$ / 7.35, 6.58, 5.48, $0.25\left(2-\mathrm{H}^{\prime} / \mathrm{o}-\mathrm{H}\right.$ of $\left.{ }^{\mathrm{a}} \mathrm{Ph}, 3-\mathrm{H}^{\prime}, 2-\mathrm{H}, \mathrm{Si}\left(\mathrm{CH}_{3}\right)_{2}\right), 5.60$ / 7.56, 6.47, 0.25 (5-H' / o-H of ' $\left.\mathrm{Ph}, 4-\mathrm{H}^{\prime}, \mathrm{Si}\left(\mathrm{CH}_{3}\right)_{2}\right), 5.48$ / 7.35, 5.64, 0.28 (2-H / o-H of ${ }^{\mathrm{a}} \mathrm{Ph}, 2-\mathrm{H}$ ', $\left.\mathrm{Si}\left(\mathrm{CH}_{3}\right)_{2}\right), 2.21$ / 7.36, 6.43 (9-H / 10-H, 16-H), 2.12 / 6.52, 1.94, 1.23, 1.19 (6-H / 4-H, 6-H', 7-H, 7-H'), 1.94 / 5.48,

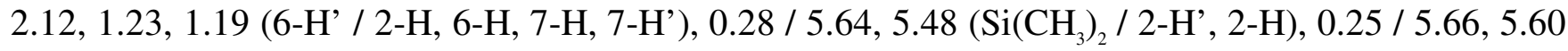
$\left(\mathrm{Si}\left(\mathrm{CH}_{3}\right)_{2} / 5-\mathrm{H}, 5-\mathrm{H}^{\prime}\right)$. GCOSY (benzene-d $\left.{ }_{6}, 600 / 600 \mathrm{MHz}\right): \delta\left({ }^{1} \mathrm{H}\right) / \delta\left({ }^{1} \mathrm{H}\right)=7.72 / 6.85(13-\mathrm{H} / 14-$ H), $7.56 / 7.26$ (o-H of ${ }^{b} \mathrm{Ph} / \mathrm{m}-\mathrm{H}$ of $\left.{ }^{b} \mathrm{Ph}\right), 7.35$ / 7.26 (o-H of ${ }^{\mathrm{a}} \mathrm{Ph} / \mathrm{m}-\mathrm{H}$ of $\left.{ }^{\mathrm{a}} \mathrm{Ph}\right), 7.26 / 7.56,7.15$ (m-H of ${ }^{b} \mathrm{Ph} / \mathrm{o}-\mathrm{H}$ of ${ }^{b} \mathrm{Ph}, \mathrm{p}-\mathrm{H}$ of $\left.{ }^{b} \mathrm{Ph}\right), 7.26 / 7.35,7.12$ (m-H of ${ }^{a} \mathrm{Ph} / \mathrm{o}-\mathrm{H}$ of ${ }^{a} \mathrm{Ph}, \mathrm{p}-\mathrm{H}$ of $\left.{ }^{a} \mathrm{Ph}\right), 7.15 / 7.26$ (p-H of ${ }^{b} \mathrm{Ph} / \mathrm{m}-\mathrm{H}$ of $\left.{ }^{b} \mathrm{Ph}\right), 7.12$ / 7.26 (p-H of ${ }^{\mathrm{a}} \mathrm{Ph} / \mathrm{m}-\mathrm{H}$ of $\left.{ }^{\mathrm{a}} \mathrm{Ph}\right), 6.85$ / $7.72(14-\mathrm{H} / 13-\mathrm{H}), 6.83 / 6.43(15-\mathrm{H}$ / 16-H), 6.58 / 6.47, 5.64 (3-H' / 4-H', 2-H'), 6.43 / 6.83 (16-H / 15-H), 6.47 / 6.58, 5.60 (4-H' / 3-H', 5-H’), 6.52 / 5.66, 5.48 (4-H / 5-H, 2-H), 5.64 / 6.58, 5.60 (2-H' / 3-H’, 5-H'), 5.66 / 6.52, 5.48 (5-H / 4-H, 2-H), 5.60 / 6.47 (5-H' / 4-H'), 5.48 / 6.52, 5.66 (2-H / 4-H, 5-H), 2.12 / 1.94, 1.23, 1.19 (6-H / 6- 
H', 7-H, 7-H'), 1.94 / 2.12, 1.23, 1.19 (6-H' / 6-H, 7-H, 7-H'), 1.49 / 1.23, 1.19 (8-H / 7-H, 7-H'), 1.23

/ 2.12, 1.94, 1.49, 1.19 ( 7-H / 6-H, 6-H', 8-H, 7-H'), 1.19 / 2.12, 1.94, 1.49, 1.23 ( 7-H / 6-H, 6-H', 8H, 7-H). GHSQC (benzene-d $\left.{ }_{6}, 150 / 600 \mathrm{MHz}\right): \delta\left({ }^{13} \mathrm{C}\right) / \delta\left({ }^{1} \mathrm{H}\right)=141.2 / 7.36(\mathrm{C} 10 / 10-\mathrm{H}), 136.3 /$ 7.56 (o-C of ${ }^{b} \mathrm{Ph} / \mathrm{o}-\mathrm{H}$ of $\left.{ }^{b} \mathrm{Ph}\right), 134.4 / 7.35$ (o-C of ${ }^{a} \mathrm{Ph} / \mathrm{o}-\mathrm{H}$ of $\left.{ }^{\mathrm{a}} \mathrm{Ph}\right), 126.5 / 7.26$ (m-C of ${ }^{\mathrm{a}} \mathrm{Ph} / \mathrm{m}-\mathrm{H}$ of $\left.{ }^{a} \mathrm{Ph}\right), 126.5 / 7.26\left(\mathrm{~m}-\mathrm{C}\right.$ of ${ }^{b} \mathrm{Ph} / \mathrm{m}-\mathrm{H}$ of $\left.{ }^{b} \mathrm{Ph}\right), 126.1 / 7.15\left(\mathrm{p}-\mathrm{C}\right.$ of ${ }^{b} \mathrm{Ph} / \mathrm{p}-\mathrm{H}$ of $\left.{ }^{b} \mathrm{Ph}\right), 125.7 / 7.12(\mathrm{p}-\mathrm{C}$ of ${ }^{\mathrm{a}} \mathrm{Ph} / \mathrm{p}-\mathrm{H}$ of $\left.{ }^{\mathrm{a}} \mathrm{Ph}\right), 125.4$ / 6.85 (C14 / 14-H), 125.1 / 6.83 (C15 / 15-H), 123.5 / 6.47 (C4' / 4-H'), 123.1 / 6.52 (C4 / 4-H), 121.8 / 6.58 (C3' / 3-H'), 116.7 / 7.72 (C13 / 13-H), 114.9 / 5.48 (C2 / 2-H), 114.3 / 5.64 (C2' / 2-H'), 113.5 / 5.66 (C5 / 5-H), 112.4 / 5.60 (C5' / 5-H'), 110.7 / 6.43 (C16 / 16-H), 33.4 / 2.12, 1.94 (C6 / 6-H, 6-H’), 30.6 / 2.21 (C9 / 9-H), 28.5 / 1.23, 1.19 (C7 / 7-H, 7-H'), 21.7 / 1.49 (C8 / 8-H), -5.8 / $0.25\left(\mathrm{Si}\left(\mathrm{CH}_{3}\right)_{2} / \mathrm{Si}\left(\mathrm{CH}_{3}\right)_{2}\right),-6.2$ / $0.28\left(\mathrm{Si}\left(\mathrm{CH}_{3}\right)_{2} / \mathrm{Si}\left(\mathrm{CH}_{3}\right)_{2}\right)$. GHMBC (benzene-d ${ }_{6}, 150$ / $600 \mathrm{MHz}): \delta\left({ }^{13} \mathrm{C}\right) / \delta\left({ }^{1} \mathrm{H}\right)=186.1 / 7.35,7.26$ (ipso-C of ${ }^{\mathrm{a}} \mathrm{Ph} / \mathrm{o}-, \mathrm{m}-\mathrm{H}$ of $\left.{ }^{\mathrm{a}} \mathrm{Ph}\right), 181.7 / 7.56,7.26$ (ipso-C of ${ }^{b} \mathrm{Ph} / \mathrm{o-}, \mathrm{m}-\mathrm{H}$ of $\left.{ }^{\mathrm{b}} \mathrm{Ph}\right), 141.2$ / 2.21 (C10 / 9-H), 138.9 / 6.52, 5.66, 5.48, 2.12, 1.94 (C3 / 4 H, 5-H, 2-H, 6-H, 6-H'), 136.3 / 7.56, 7.15 (o-C of ${ }^{b} \mathrm{Ph} /$ o-, p-H of ${ }^{\mathrm{b}} \mathrm{Ph}$ ), 136.3 / 7.36, 6.85, 6.43 (C12 / 10-H, 14-H, 16-H), 134.4 / 7.35, 7.12 (o-C of ${ }^{\mathrm{a}} \mathrm{Ph} / \mathrm{o}-, \mathrm{p}-\mathrm{H}$ of $\left.{ }^{\mathrm{a}} \mathrm{Ph}\right), 132.7$ / 7.72, 7.36, 6.83, 2.21 (C12 / 13-H, 10-H, 15-H, 9-H), 126.5 / 7.26 (m-C of ${ }^{\mathrm{a}} \mathrm{Ph},{ }^{\mathrm{b}} \mathrm{Ph} / \mathrm{m}-\mathrm{H}$ of $\left.{ }^{\mathrm{a}} \mathrm{Ph},{ }^{\mathrm{b}} \mathrm{Ph}\right), 126.1 / 7.56\left(\mathrm{p}-\mathrm{C}\right.$ of ${ }^{\mathrm{b}} \mathrm{Ph} / \mathrm{o}-$ $\mathrm{H}$ of $\left.{ }^{b} \mathrm{Ph}\right), 125.7$ / 7.35 (p-C of ${ }^{a} \mathrm{Ph} / \mathrm{o}-\mathrm{H}$ of $\left.{ }^{\mathrm{a}} \mathrm{Ph}\right), 125.4$ / 6.43 (C14 / 16-H), 125.1 / 7.72 (C15 / 13-H), 123.5 / 5.64 (C4' / 2-H'), 123.1 / 5.48, 1.94 (C4 / 2-H, 6-H'), 121.8 / 5.60 (C3' / 5-H'), 116.7 / 6.83 (C13 / 15-H), 114.9 / 6.52, 5.66, 2.12 (C2 / 4-H, 5-H, 6-H), 114.3 / 5.60 (C2' / 5-H'), 113.5 / 6.52 , 5.48 (C5 / 4-H, 2-H), 112.4 / 5.64 (C5' / 2-H'), 110.7 / 6.85 (C16 / 14-H), 100.5 / 6.52, 5.66, 5.48, 0.28, 0.25 (C1 / 4-H, 2-H, 5-H', 5-H, Si( $\left.\left.\mathrm{CH}_{3}\right)_{2}\right), 100.1$ / 5.64, 5.60, 0.28, 0.25 (C1' / 2-H', 2-H, $\left.\mathrm{Si}\left(\mathrm{CH}_{3}\right)_{2}\right), 33.4$ / 1.23, 1.19 (C6 / 7-H, 7-H'), 28.5 / 2.12, 1.94, 1.49 (C7 / 6-H, 6-H', 8-H), -5.8 / 0.28 $\left(\mathrm{Si}\left(\mathrm{CH}_{3}\right)_{2} / \mathrm{Si}\left(\mathrm{CH}_{3}\right)_{2}\right),-6.2 / 0.25\left(\mathrm{Si}\left(\mathrm{CH}_{3}\right)_{2} / \mathrm{Si}\left(\mathrm{CH}_{3}\right)_{2}\right)$.

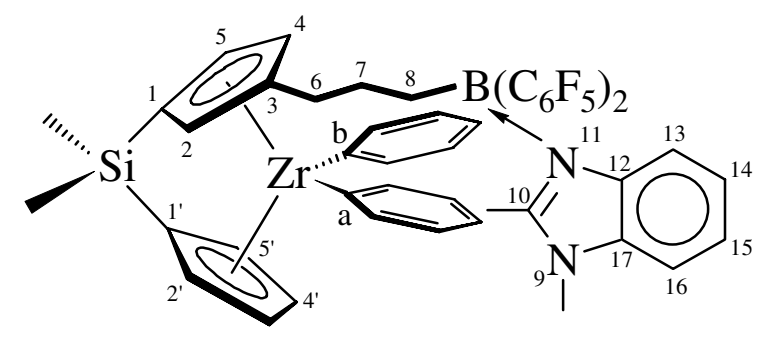

Reaction of complex 12 with 1,2dimethylbenzimidazole, preparation of $19 \mathrm{~b}$. $\mathrm{mp}$ 
$170^{\circ} \mathrm{C}$ (decomp.). Anal. Calcd. for $\mathrm{C}_{48} \mathrm{H}_{39} \mathrm{~N}_{2} \mathrm{BF}_{10} \mathrm{SiZr}$ (964.0): C 59.81, H 4.08, N 2.91; found: C 59.69, H 4.27, N 2.79\%. IR (KBr): $\tilde{v}=3042$ (w), 2909 (w), 1630 (m), $1511(\mathrm{~s}), 1449$ (s), 1274 (m), 1245 (m), 1155 (w), 1092 (s), 966 (s), 805 (s), 721 (s), 701 (s), 677 (s), 554 (w), 463 (m) cm ${ }^{-1} .{ }^{1} \mathrm{H}-\mathrm{NMR}$ (benzene-d $\left.{ }_{6}, 600 \mathrm{MHz}\right): \delta=7.67(\mathrm{~m}, 1 \mathrm{H}, 13-\mathrm{H}), 7.53,7.22,7.14$ (each m, 5H, o-, m-, p-H of ${ }^{\mathrm{b}} \mathrm{Ph}$ ), 7.33, 7.21, 7.07 (each m, 5H, o-, m-, p-H of $\left.{ }^{\mathrm{a}} \mathrm{Ph}\right), 6.92(\mathrm{~m}, 1 \mathrm{H}, 14-\mathrm{H}), 6.90(\mathrm{~m}, 1 \mathrm{H}, 15-\mathrm{H}), 6.54(\mathrm{~m}, 1 \mathrm{H}$, 3-H'), 6.46 (m, 1H, 16-H), 6.45 (m, 1H, 4-H'), 6.43 (m, 1H, 4-H), 5.63 (m, 1H, 2-H'), 5.61 (m, 1H, 5H), 5.55 (m, 1H, 5-H'), 5.43 (m, 1H, 2-H), 2.09/1.99 (each m, each 1H, 6-H, 6-H'), 2.08 (s, 3H, 9-H), $1.75\left(\mathrm{~s}, 3 \mathrm{H}, \mathrm{Me}_{10}-\mathrm{H}\right), 1.59$ (m, 2H, 8-H), 1.23/1.15 (each m, each 1H, 7-H, 7-H'), 0.25/0.23 (each s, each $\left.3 \mathrm{H}, \mathrm{Si}\left(\mathrm{CH}_{3}\right)_{2}\right) .{ }^{13} \mathrm{C}\left\{{ }^{1} \mathrm{H}\right\}-\mathrm{NMR}$ (benzene-d, $150 \mathrm{MHz}$ ): $\delta=186.2,134.3,126.8,125.6$ (ipso-, o-, m-, p-C of $\left.{ }^{\mathrm{a}} \mathrm{Ph}\right), 181.7,136.4,126.3,125.8$ (ipso-, o-, m-, p-C of $\left.{ }^{\mathrm{b}} \mathrm{Ph}\right), 151.8$ (C10), 138.5 (C3), 136.2 (C12), 132.1 (C17), 124.5 (C14), 124.2 (C15), 123.6 (C4'), 123.2 (C4), 121.1 (C3'), 117.7 (C13), 114.8 (C2), 114.3 (C2'), 113.2 (C5), 112.1 (C5'), 110.0 (C16), 100.3 (C1, C1'), 33.2 (C6), 28.9 (C7), 28.8 (C9), 22.9 (C8), $11.8\left(\mathrm{CMe}_{10}\right),-5.3 /-5.6\left(\mathrm{Si}\left(\mathrm{CH}_{3}\right)_{2}\right) ; 148.5\left({ }^{1} \mathrm{~J}_{\mathrm{CF}}=240.4 \mathrm{~Hz}\right.$, o-C of $\left.\mathrm{C}_{6} \mathrm{~F}_{5}\right), 139.5$ $\left({ }^{1} \mathrm{~J}_{\mathrm{CF}}=248.3 \mathrm{~Hz}, \mathrm{p}-\mathrm{C}\right.$ of $\left.\mathrm{C}_{6} \mathrm{~F}_{5}\right), 137.5\left({ }^{1} \mathrm{~J}_{\mathrm{CF}}=251.1 \mathrm{~Hz}, \mathrm{~m}-\mathrm{C}\right.$ of $\left.\mathrm{C}_{6} \mathrm{~F}_{5}\right), 122.7$ (ipso-C of $\left.\mathrm{C}_{6} \mathrm{~F}_{5}\right) .{ }^{11} \mathrm{~B}\left\{{ }^{1} \mathrm{H}\right\}-$ NMR (benzene-d $\left.{ }_{6}, 64 \mathrm{MHz}\right): \delta=-3.30\left(v_{1 / 2}=416 \mathrm{~Hz}\right) .{ }^{19} \mathrm{~F}-\mathrm{NMR}\left(\right.$ benzene-d $\left._{6}, 564 \mathrm{MHz}\right): \delta=-132.1$ $\left(\mathrm{m}, 4 \mathrm{~F}, \mathrm{o}-\mathrm{F}\right.$ of $\left.\mathrm{C}_{6} \mathrm{~F}_{5}\right),-158.5\left(\mathrm{t},{ }^{3} \mathrm{~J}_{\mathrm{FF}}=22.0 \mathrm{~Hz}, 1 \mathrm{~F}, \mathrm{p}-\mathrm{F}\right.$ of $\left.\mathrm{C}_{6} \mathrm{~F}_{5}\right),-158.6\left(\mathrm{t},{ }^{3} \mathrm{~J}_{\mathrm{FF}}=22.0 \mathrm{~Hz}, 1 \mathrm{~F}, \mathrm{p}-\mathrm{F}^{\prime}\right.$ of $\left.\mathrm{C}_{6} \mathrm{~F}_{5}\right),-163.7\left(\mathrm{~m}, 4 \mathrm{~F}, \mathrm{~m}-\mathrm{F}\right.$ of $\left.\mathrm{C}_{6} \mathrm{~F}_{5}\right) .1 \mathrm{D}-\mathrm{TOCSY}\left(\right.$ benzene-d $\left.\mathrm{d}_{6}, 600 / 600 \mathrm{MHz}\right): \delta\left({ }^{1} \mathrm{H}\right)_{\text {irr. }} / \delta\left({ }^{1} \mathrm{H}\right)_{\text {resp. }}=7.67$ / 6.92, 6.90, 6.46 (13-H / 14-H, 15-H, 16-H), 7.53 / 7.22, 7.14 (o-H of ${ }^{b} \mathrm{Ph} / \mathrm{m}-, \mathrm{p}-\mathrm{H}$ of $\left.{ }^{\mathrm{b}} \mathrm{Ph}\right), 7.33$ / 7.21, 7.07 (o-H of ${ }^{a} \mathrm{Ph} / \mathrm{m}-$, p-H of $\left.{ }^{\mathrm{a}} \mathrm{Ph}\right), 5.55$ / 6.54, 6.45, 5.63 (5-H' / 3-H', 4-H', 2-H'), 5.43 / 6.43, 5.61 (2-H / 4-H, 5-H), 1.59 / 2.09, 1.99, 1.23, 1.15 (8-H / 6-H, 6-H', 7-H, 7-H'). NOE-Diff (benzene$\left.\mathrm{d}_{6}, 600 / 600 \mathrm{MHz}\right): \delta\left({ }^{1} \mathrm{H}\right)_{\text {irr. }} / \delta\left({ }^{1} \mathrm{H}\right)_{\text {resp. }}=7.67 / 6.92,1.59,1.23,1.15\left(13-\mathrm{H} / 14-\mathrm{H}, 8-\mathrm{H}, 7-\mathrm{H}, 7-\mathrm{H}^{\prime}\right)$, 7.53 / 7.33, 7.22, 6.43, 5.61, 5.55 (o-H of ${ }^{b} \mathrm{Ph} / \mathrm{o}-\mathrm{H}$ of ${ }^{\mathrm{a}} \mathrm{Ph}, \mathrm{m}-\mathrm{H}$ of $\left.{ }^{\mathrm{b}} \mathrm{Ph}, 4-\mathrm{H}, 5-\mathrm{H}, 5-\mathrm{H}^{\prime}\right), 7.33$ / 7.53 , 7.21, 6.54, 5.63, 5.43 (o-H of ${ }^{a} \mathrm{Ph} / \mathrm{o}-\mathrm{H}$ of ${ }^{\mathrm{b}} \mathrm{Ph}, \mathrm{m}-\mathrm{H}$ of ${ }^{\mathrm{a}} \mathrm{Ph}, 3-\mathrm{H}$ ', 2-H', 2-H), 7.07 / 7.22 (p-H of ${ }^{\mathrm{a}} \mathrm{Ph} /$ m-H of $\left.{ }^{\mathrm{a}} \mathrm{Ph}\right), 6.54$ / 7.33, 5.63 (3-H' / o-H of $\left.{ }^{\mathrm{a}} \mathrm{Ph}, 2-\mathrm{H}^{\prime}\right), 6.46$ / 6.90, 2.08 (16-H / 15-H, 9-H), 6.45 / 7.53, 6.54, 5.55 (4-H' / o-H of $\left.{ }^{b} \mathrm{Ph}, 3-\mathrm{H}^{\prime}, 5-\mathrm{H}^{\prime}\right), 6.43$ / 7.53 (4-H / o-H of $\left.{ }^{\mathrm{b}} \mathrm{Ph}\right), 5.63$ / 7.33, 6.54, 0.23 
(2-H' / o-H of $\left.{ }^{\mathrm{a}} \mathrm{Ph}, 3-\mathrm{H}^{\prime}, \mathrm{Si}\left(\mathrm{CH}_{3}\right)_{2}\right), 5.61 / 7.53,6.43,0.23\left(5-\mathrm{H} / \mathrm{o}-\mathrm{H}\right.$ of $\left.{ }^{\mathrm{b}} \mathrm{Ph}, 4-\mathrm{H}, \mathrm{Si}\left(\mathrm{CH}_{3}\right)_{2}\right), 5.55$ / 7.53, 6.45, 0.23 (5-H' / o-H of $\left.{ }^{b} \mathrm{Ph}, 4-\mathrm{H}^{\prime}, \mathrm{Si}\left(\mathrm{CH}_{3}\right)_{2}\right), 5.43$ / 7.33, 0.25 (2-H / o-H of $\left.{ }^{\mathrm{a}} \mathrm{Ph}, \mathrm{Si}\left(\mathrm{CH}_{3}\right)_{2}\right), 2.09$ / 6.43, 1.99, 1.23, 1.15 (6-H / 4-H, 6-H', 7-H, 7-H'), 2.08 / 6.46, 1.75 (9-H / 16-H, Me $\left.{ }_{10}-\mathrm{H}\right), 1.99$ / 5.43, 2.09, 1.23, 1.15 (6-H' / 2-H, 6-H, 7-H, 7-H'), 1.75 / $2.08\left(\mathrm{Me}_{10}-\mathrm{H} / 9-\mathrm{H}\right), 0.25$ / 5.63, 5.43 $\left(\mathrm{Si}\left(\mathrm{CH}_{3}\right)_{2} / 2-\mathrm{H}^{\prime}, 2-\mathrm{H}\right), 0.23$ / 5.61, $5.55\left(\mathrm{Si}\left(\mathrm{CH}_{3}\right)_{2} /\right.$ 5-H, 5-H'). GCOSY (benzene-d, 600 / $\left.600 \mathrm{MHz}\right)$ : $\delta\left({ }^{1} \mathrm{H}\right) / \delta\left({ }^{1} \mathrm{H}\right)=7.67 / 6.92(13-\mathrm{H} / 14-\mathrm{H}), 7.53 / 7.22\left(\mathrm{o}-\mathrm{H}\right.$ of ${ }^{b} \mathrm{Ph} / \mathrm{m}-\mathrm{H}$ of $\left.{ }^{b} \mathrm{Ph}\right), 7.33 / 7.21\left(\mathrm{o}-\mathrm{H}\right.$ of ${ }^{\mathrm{a}} \mathrm{Ph}$ / m-H of $\left.{ }^{a} \mathrm{Ph}\right), 7.22 / 7.53,7.14\left(\mathrm{~m}-\mathrm{H}\right.$ of ${ }^{b} \mathrm{Ph} / \mathrm{o}-\mathrm{H}$ of ${ }^{b} \mathrm{Ph}, \mathrm{p}-\mathrm{H}$ of $\left.{ }^{b} \mathrm{Ph}\right), 7.21 / 7.33,7.07$ (m-H of ${ }^{\mathrm{a}} \mathrm{Ph} / \mathrm{o}-$ $\mathrm{H}$ of ${ }^{a} \mathrm{Ph}, \mathrm{p}-\mathrm{H}$ of $\left.{ }^{\mathrm{a}} \mathrm{Ph}\right), 7.14$ / $7.22\left(\mathrm{p}-\mathrm{H}\right.$ of ${ }^{b} \mathrm{Ph} / \mathrm{m}-\mathrm{H}$ of $\left.{ }^{b} \mathrm{Ph}\right), 7.07 / 7.21$ (p-H of ${ }^{a} \mathrm{Ph} / \mathrm{m}-\mathrm{H}$ of $\left.{ }^{a} \mathrm{Ph}\right), 6.92$ / 7.67 (14-H / 13-H), 6.90 / 6.46 (15-H / 16-H), 6.54 / 6.45, 5.63 (3-H' / 4-H', 2-H'), 6.46 / 6.90 (16-H / 15-H), 6.45 / 6.54, 5.55 (4-H' / 3-H', 5-H’), 6.43 / 5.61, 5.43 (4-H / 5-H, 2-H), 5.63 / 6.54, 5.55 (2-H’ / 3-H', 5-H'), 5.61 / 6.43, 5.43 (5-H / 4-H, 2-H), 5.55 / 6.45 (5-H' / 4-H’), 5.43 / 6.43, 5.61 (2-H / 4-H, 5-H), 2.09 / 1.99, 1.23, 1.15 (6-H / 6-H’, 7-H, 7-H’), 1.99 / 2.09, 1.23, 1.15 (6-H’ / 6-H, 7-H, 7-H'), 1.59 / 1.23, 1.15 (8-H / 7-H, 7-H'), 1.23 / 2.09, 1.99, 1.59, 1.15 ( 7-H / 6-H, 6-H', 8-H, 7-H'), 1.15 / 2.09, 1.99, 1.59, 1.23 ( 7-H / 6-H, 6-H', 8-H, 7-H). GHSQC (benzene-d, $150 / 600 \mathrm{MHz}): \delta\left({ }^{13} \mathrm{C}\right) / \delta$ $\left({ }^{1} \mathrm{H}\right)=136.4 / 7.53\left(\mathrm{o}-\mathrm{C}\right.$ of ${ }^{\mathrm{b}} \mathrm{Ph} / \mathrm{o}-\mathrm{H}$ of $\left.{ }^{\mathrm{b}} \mathrm{Ph}\right), 134.3 / 7.33\left(\mathrm{o}-\mathrm{C}\right.$ of ${ }^{\mathrm{a}} \mathrm{Ph} / \mathrm{o}-\mathrm{H}$ of $\left.{ }^{\mathrm{a}} \mathrm{Ph}\right), 126.8 / 7.21(\mathrm{~m}-\mathrm{C}$ of ${ }^{a} \mathrm{Ph} / \mathrm{m}-\mathrm{H}$ of $\left.{ }^{\mathrm{a}} \mathrm{Ph}\right), 126.3 / 7.22\left(\mathrm{~m}-\mathrm{C}\right.$ of ${ }^{b} \mathrm{Ph} / \mathrm{m}-\mathrm{H}$ of $\left.{ }^{b} \mathrm{Ph}\right), 125.8 / 7.14\left(\mathrm{p}-\mathrm{C}\right.$ of ${ }^{b} \mathrm{Ph} / \mathrm{p}-\mathrm{H}$ of $\left.{ }^{b} \mathrm{Ph}\right)$, 125.6 / 7.07 (p-C of ${ }^{\mathrm{a}} \mathrm{Ph} / \mathrm{p}-\mathrm{H}$ of $\left.{ }^{\mathrm{a}} \mathrm{Ph}\right), 124.5$ / 6.92 (C14 / 14-H), 124.2 / 6.90 (C15 / 15-H), 123.6 / 6.45 (C4' / 4-H'), 123.2 / 6.43 (C4 / 4-H), 121.1 / 6.54 (C3' / 3-H'), 117.7 / 7.67 (C13 / 13-H), 114.8 / 5.43 (C2 / 2-H), 114.3 / 5.63 (C2' / 2-H'), 113.2 / 5.61 (C5 / 5-H), 112.1 / 5.55 (C5' / 5-H'), 110.0 / 6.46 (C16 / 16-H), 33.2 / 2.09, 1.99 (C6 / 6-H, 6-H'), 28.8 / 2.08 (C9 / 9-H), 28.9 / 1.23, 1.15 (C7 / 7 H, 7-H'), 22.9 / $1.59(\mathrm{C} 8 / 8-\mathrm{H}), 11.8$ / $\left.1.75\left(\mathrm{CMe}_{10} / \mathrm{Me}_{10}-\mathrm{H}\right),-5.3 / 0.23\left(\mathrm{Si}_{\left(\mathrm{CH}_{3}\right.}\right)_{2} / \mathrm{Si}\left(\mathrm{CH}_{3}\right)_{2}\right),-5.6 /$ $0.25\left(\mathrm{Si}\left(\mathrm{CH}_{3}\right)_{2} / \mathrm{Si}\left(\mathrm{CH}_{3}\right)_{2}\right)$. GHMBC (benzene-d, $\left.150 / 600 \mathrm{MHz}\right): \delta\left({ }^{13} \mathrm{C}\right) / \delta\left({ }^{1} \mathrm{H}\right)=186.2 / 7.33,7.21$ (ipso-C of ${ }^{a} \mathrm{Ph} / \mathrm{o-}, \mathrm{m}-\mathrm{H}$ of ${ }^{\mathrm{a}} \mathrm{Ph}$ ), 181.7 / 7.53, 7.22 (ipso-C of ${ }^{b} \mathrm{Ph} / \mathrm{o}-, \mathrm{m}-\mathrm{H}$ of ${ }^{\mathrm{b}} \mathrm{Ph}$ ), $151.8 / 2.08,1.75$ (C10 / 9-H, $\left.\mathrm{Me}_{10}-\mathrm{H}\right), 138.5$ / 6.43, 5.61, 5.43, 2.09, 1.99 (C3 / 4-H, 5-H, 2-H, 6-H, 6-H'), 136.4 / 7.53, 7.14 (o-C of ${ }^{b} \mathrm{Ph} / \mathrm{o}-, \mathrm{p}-\mathrm{H}$ of $\left.{ }^{\mathrm{b}} \mathrm{Ph}\right), 136.2$ / 6.92, 6.46 (C12 / 14-H, 16-H), 134.3 / 7.33, 7.07 (o-C of ${ }^{\mathrm{a}} \mathrm{Ph}$ / o-, p-H of $\left.{ }^{\mathrm{a}} \mathrm{Ph}\right), 132.1 / 7.67,6.90,2.08$ (C12 / 13-H, 15-H, 9-H), 126.8 / 7.21 (m-C of ${ }^{\mathrm{a}} \mathrm{Ph} / \mathrm{m}-\mathrm{H}$ of 
$\left.{ }^{a} \mathrm{Ph}\right), 126.3 / 7.22\left(\mathrm{~m}-\mathrm{C}\right.$ of ${ }^{\mathrm{b}} \mathrm{Ph} / \mathrm{m}-\mathrm{H}$ of $\left.{ }^{\mathrm{b}} \mathrm{Ph}\right), 125.8 / 7.53\left(\mathrm{p}-\mathrm{C}\right.$ of ${ }^{\mathrm{b}} \mathrm{Ph} / \mathrm{o}-\mathrm{H}$ of $\left.{ }^{\mathrm{b}} \mathrm{Ph}\right), 125.6 / 7.33(\mathrm{p}-\mathrm{C}$ of ${ }^{\mathrm{a}} \mathrm{Ph} / \mathrm{o}-\mathrm{H}$ of $\left.{ }^{\mathrm{a}} \mathrm{Ph}\right), 124.5$ / 6.46 (C14 / 16-H), 124.2 / 7.67 (C15 / 13-H), 123.6 / 5.63 (C4' / 2-H'), 123.2 / 5.43, 1.99 (C4 / 2-H, 6-H'), 121.1 / 5.55 (C3' / 5-H'), 117.7 / 6.90 (C13 / 15-H), 114.8 / 6.43, 5.61, 2.09 (C2 / 4-H, 5-H, 6-H), 114.3 / 5.55 (C2' / 5-H'), 113.2 / 6.43, 5.43 (C5 / 4-H, 2-H), 112.1 / 5.63 (C5' / 2-H'), 110.0 / 6.92 (C16 / 14-H), 100.3 / 6.43, 5.63, 5.61, 5.55, 5.43, 0.25, 0.23 (C1, C1' / 4-H, 2-H', 2-H, 5-H', 5-H, Si( $\left.\left.\mathrm{CH}_{3}\right)_{2}\right)$, 33.2 / 1.59, 1.23, 1.15 (C6 / 8-H, 7-H, 7-H'), 28.9 / 2.09, 1.99, 1.59 (C7 / 6-H, 6-H', 8-H), 22.9 / 2.09, 1.99 (C8 / 6-H, 6-H'), -5.3 / $0.25\left(\mathrm{Si}\left(\mathrm{CH}_{3}\right)_{2} / \mathrm{Si}\left(\mathrm{CH}_{3}\right)_{2}\right),-5.6 / 0.23$ $\left(\mathrm{Si}\left(\mathrm{CH}_{3}\right)_{2} / \mathrm{Si}\left(\mathrm{CH}_{3}\right)_{2}\right)$.

Thermolysis of 19a in the presence of 1-methylbenzimidazole, formation of 20 . $(20 \mathrm{~A}: 20 \mathrm{~B}=$ 3:2), $\mathrm{mp} 282^{\circ} \mathrm{C}$ (decomp.). Anal. Calcd. for $\mathrm{C}_{49} \mathrm{H}_{39} \mathrm{~N}_{4} \mathrm{BF}_{10} \mathrm{SiZr}$ (1004.0): C 58.62, H 3.92, N 5.58; found C 59.35, H 4.04, N 4.03\%. IR (KBr): $\widetilde{v}=3042(\mathrm{w}), 2910(\mathrm{w}), 1644(\mathrm{~m}), 1539(\mathrm{~m}), 1504(\mathrm{~s}), 1456(\mathrm{~s})$, $1246(\mathrm{~m}), 1197(\mathrm{~m}), 1085(\mathrm{~m}), 952(\mathrm{~m}), 791(\mathrm{~m}), 742(\mathrm{~m}), 672(\mathrm{~m}), 463(\mathrm{w}) \mathrm{cm}^{-1}$.

Diastereoisomer 20A: ${ }^{1} \mathrm{H}-\mathrm{NMR}$ (benzene-d 6 , $600 \mathrm{MHz}$ ): $\delta=8.29,7.52,7.33$ (each m, 5H, o-, m-, p-

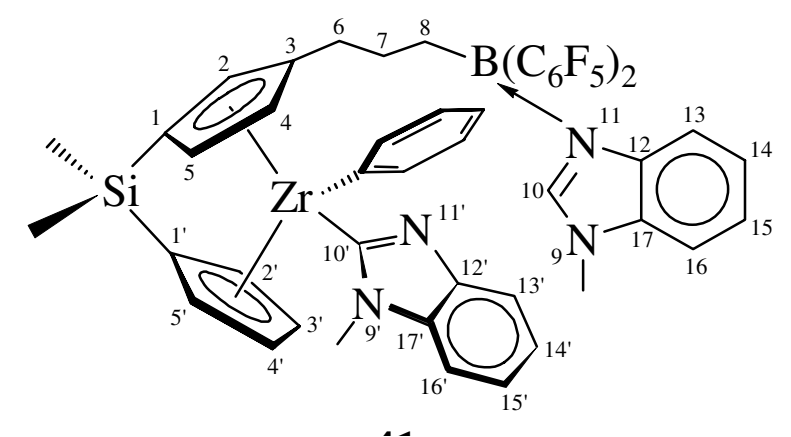

$\mathrm{H}$ of $\mathrm{Ph}), 8.01\left(\mathrm{~m}, 1 \mathrm{H}, 13-\mathrm{H}^{\prime}\right), 7.69(\mathrm{~m}, 1 \mathrm{H}, 13-\mathrm{H})$, $7.32(\mathrm{~s}, 1 \mathrm{H}, 10-\mathrm{H}), 7.14\left(\mathrm{~m}, 1 \mathrm{H}, 15-\mathrm{H}^{\prime}\right), 7.12(\mathrm{~m}, 1 \mathrm{H}$, 14-H'), 6.97 (m, 1H, 16-H'), 6.83 (m, 2H, 14-H, 15H), 6.57 (m, 1H, 3-H'), $6.46(\mathrm{~m}, 1 \mathrm{H}, 16-\mathrm{H}), 6.16$ (m, 1H, 5-H), $6.08\left(\mathrm{~m}, 1 \mathrm{H}, 5-\mathrm{H}^{\prime}\right), 5.84\left(\mathrm{~m}, 1 \mathrm{H}, 2-\mathrm{H}^{\prime}\right)$, $5.55(\mathrm{~m}, 1 \mathrm{H}, 2-\mathrm{H}), 5.13\left(\mathrm{~m}, 1 \mathrm{H}, 4-\mathrm{H}^{\prime}\right), 5.09(\mathrm{~m}, 1 \mathrm{H}, 4-\mathrm{H}), 3.37$ (s, 3H, 9-H'), 2.31 (s, 3H, 9-H), 1.97/1.94 (each m, each 1H, 6-H, 6-H'), 1.45 (m, 2H, 8-H), 1.14 (m, 2H, 7-H), 0.46/0.44 (each s, each $\left.3 \mathrm{H}, \mathrm{Si}\left(\mathrm{CH}_{3}\right)_{2}\right) .{ }^{13} \mathrm{C}\left\{{ }^{1} \mathrm{H}\right\}-\mathrm{NMR}$ (benzene-d $\mathrm{b}_{6}, 150 \mathrm{MHz}$ ): $\delta=181.4,142.0,127.0,124.1$ (ipso-, o-, m-, p-C of Ph), 202.9 (C10'), 143.6 (C17'), 141.2 (C10), 140.8 (C12'), 140.6 (C3), 136.4 (C12), 132.7 (C17), 127.3 (C14'), 125.4 (C14), 125.1 (C15), 122.9 (C3’), 122.7 (C15'), 118.4 (C13'), 116.7 (C13), 114.4 (C4), 114.0 (C4'), 110.9 (C16), 109.8 (C16'), 108.9 (C5), 108.8 (C2), 108.5 (C5'), 108.4 (C2'), 102.9 
(C1), 102.8 (C1'), 33.3 (C6), 32.6 (C9'), 30.8 (C9), 28.7 (C7), 21.6 (C8), -4.2/-5.9 ( $\left.\mathrm{Si}\left(\mathrm{CH}_{3}\right)_{2}\right) ; 148.5$ $\left({ }^{1} \mathrm{~J}_{\mathrm{CF}}=238.4 \mathrm{~Hz}, \mathrm{o}-\mathrm{C}\right.$ of $\left.\mathrm{C}_{6} \mathrm{~F}_{5}\right), 139.4\left({ }^{1} \mathrm{~J}_{\mathrm{CF}}=251.5 \mathrm{~Hz}, \mathrm{p}-\mathrm{C}\right.$ of $\left.\mathrm{C}_{6} \mathrm{~F}_{5}\right), 137.5\left({ }^{1} \mathrm{~J}_{\mathrm{CF}}=249.6 \mathrm{~Hz}, \mathrm{~m}-\mathrm{C}\right.$ of $\left.\mathrm{C}_{6} \mathrm{~F}_{5}\right), 121.7$ (ipso-C of $\left.\mathrm{C}_{6} \mathrm{~F}_{5}\right) .{ }^{11} \mathrm{~B}\left\{{ }^{1} \mathrm{H}\right\}-\mathrm{NMR}$ (benzene- $\left.\mathrm{d}_{6}, 64 \mathrm{MHz}\right): \delta=-5.10\left(v_{1 / 2}=341 \mathrm{~Hz}\right) .{ }^{19} \mathrm{~F}-\mathrm{NMR}$ (benzene- $\left.\mathrm{d}_{6}, 564 \mathrm{MHz}\right): \delta=-132.9\left(\mathrm{~m}, 2 \mathrm{~F}, \mathrm{o}-\mathrm{F}\right.$ of $\left.\mathrm{C}_{6} \mathrm{~F}_{5}\right),-133.2\left(\mathrm{~m}, 2 \mathrm{~F}, \mathrm{o}-\mathrm{F}\right.$ ' of $\left.\mathrm{C}_{6} \mathrm{~F}_{5}\right),-158.7\left(\mathrm{t},{ }^{3} \mathrm{~J}_{\mathrm{FF}}=\right.$ $20.8 \mathrm{~Hz}, 1 \mathrm{~F}, \mathrm{p}-\mathrm{F}$ of $\left.\mathrm{C}_{6} \mathrm{~F}_{5}\right),-158.8\left(\mathrm{t},{ }^{3} \mathrm{~J}_{\mathrm{FF}}=20.8 \mathrm{~Hz}, 1 \mathrm{~F}, \mathrm{p}-\mathrm{F}\right.$ ' of $\left.\mathrm{C}_{6} \mathrm{~F}_{5}\right),-164.0\left(\mathrm{~m}, 2 \mathrm{~F}, \mathrm{~m}-\mathrm{F}\right.$ of $\left.\mathrm{C}_{6} \mathrm{~F}_{5}\right),-$ $164.1\left(\mathrm{~m}, 2 \mathrm{~F}, \mathrm{~m}-\mathrm{F}^{\prime}\right.$ of $\left.\mathrm{C}_{6} \mathrm{~F}_{5}\right) .1 \mathrm{D}-\mathrm{TOCSY}\left(\right.$ benzene-d $\left.\mathrm{d}_{6}, 600 / 600 \mathrm{MHz}\right): \delta\left({ }^{1} \mathrm{H}\right)_{\text {irr. }} / \delta\left({ }^{1} \mathrm{H}\right)_{\text {resp. }}=8.29 /$ 7.52, 7.33 (o-H of Ph / m-, p-H of Ph), 8.01 / 7.14, 7.12, 6.97 (13-H' / 15-H', 14-H', 16-H'), 7.69 / 6.83, 6.46 (13-H / 14-H, 15-H, 16-H), 6.57 / 5.13, 6.08, 5.84 (3-H' / 4-H', 5-H', 2-H'), 5.55 / 5.19, 6.16 (2-H / 4-H, 5-H), 1.97, 1.94 / 1.45, 1.14 (6-H, 6-H' / 8-H, 7-H). NOE-Diff (benzene-d 6 , 600 / 600 $\mathrm{MHz}): \delta\left({ }^{1} \mathrm{H}\right)_{\text {irr. }} / \delta\left({ }^{1} \mathrm{H}\right)_{\text {resp. }}=8.29 / 8.01,7.52,6.57,5.84,5.55\left(\mathrm{o}-\mathrm{H}\right.$ of $\mathrm{Ph} / 13-\mathrm{H}^{\prime}, \mathrm{m}-\mathrm{H}$ of $\mathrm{Ph}, 3-\mathrm{H}^{\prime}, 2-$ H', 2-H), 8.01 / 7.12 (13-H' / 14-H'), 7.52 / 8.29, 7.33 (m-H of Ph / o-, p-H of Ph), 6.83 / 7.69, 6.46 (14-H, 15-H / 13-H, 16-H), 6.57 / 8.29, 5.84, 5.13 (3-H' / o-H of Ph, 2-H', 4-H'), 6.46 / 6.83, 2.31 (16H / 15-H, 9-H), 6.16 / 8.29, 5.09, 3.37, 0.46 (5-H / o-H of Ph, 4-H, 9-H', $\left.\mathrm{Si}\left(\mathrm{CH}_{3}\right)_{2}\right), 6.08$ / 5.13, 3.37, 0.46 (5-H' / 4-H', 9-H', $\left.\mathrm{Si}\left(\mathrm{CH}_{3}\right)_{2}\right), 5.84$ / 8.29, 6.57, 0.44 (2-H' / o-H of Ph, 3-H', $\left.\mathrm{Si}\left(\mathrm{CH}_{3}\right)_{2}\right), 5.55$ / 8.29, 1.97, 1.94, 0.44 (2-H / o-H of Ph, 6-H, 6-H', $\left.\left.\mathrm{Si}_{(} \mathrm{CH}_{3}\right)_{2}\right)$, 5.13 / 6.57, 6.08, 3.37 (4-H' / 3-H', 5-H', 9-H'), 5.09 / 6.16, 6.08, 3.37, 1.97, 1.94, 1.14 (4-H / 5-H, 5-H', 9-H', 6-H, 6-H', 7-H), 3.37 / 8.29, 6.97, 6.16, 6.08, 0.46 (9-H' / o-H of Ph, 16-H', 5-H, 5-H', $\left.\mathrm{Si}\left(\mathrm{CH}_{3}\right)_{2}\right), 1.97,1.94$ / 8.29, 5.55, 5.09, 1.14 (6-H, 6-H' / o-H of Ph, 2-H, 4-H, 7-H), 0.46 / 6.16, $\left.6.08\left(\mathrm{Si}^{(} \mathrm{CH}_{3}\right)_{2} / 5-\mathrm{H}, 5-\mathrm{H}^{\prime}\right), 0.44$ / 5.84, 5.55 $\left(\mathrm{Si}\left(\mathrm{CH}_{3}\right)_{2} / 2-\mathrm{H}^{\prime}, 2-\mathrm{H}\right)$. GCOSY (benzene-d $\left.{ }_{6}, 600 / 600 \mathrm{MHz}\right): \delta\left({ }^{1} \mathrm{H}\right) / \delta\left({ }^{1} \mathrm{H}\right)=8.29 / 7.52,7.33(\mathrm{o}-\mathrm{H}$ of $\mathrm{Ph} / \mathrm{m}-\mathrm{H}$ of $\mathrm{Ph}, \mathrm{p}-\mathrm{H}$ of Ph), 8.01 / 7.12 (13-H' / 14-H'), 7.69 / 6.83 (13-H / 15-H, 14-H), 7.52 / 8.29, 7.33 (m-H of Ph / o-H of Ph, p-H of Ph), 7.33 / 7.52 (p-H of Ph / m-H of Ph), 7.32 / 2.31 (10-H / 9-H),

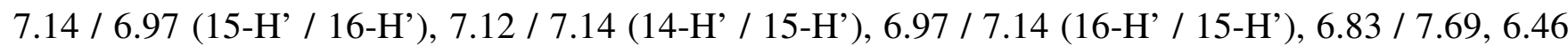
(14-H, 15-H / 13-H, 16-H), 6.57 / 5.84, 5.13 (3-H' / 2-H', 4-H'), 6.46 / 6.83 (16-H / 14-H, 15-H), 6.16 / 5.55, 5.09 (5-H / 2-H, 4-H), 6.08 / 6.57, 5.84, 5.13 (5-H' / 3-H', 2-H’, 4-H'), 5.84 / 6.57, 6.08, 5.13 (2-H' / 3-H', 5-H', 4-H'), 5.55 / 6.16, 5.09 (2-H / 5-H, 4-H), 5.13 / 6.57, 6.08 (4-H’ / 3-H', 5-H’), 5.09 
/ 6.16, 5.55 (4-H / 5-H, 2-H), 2.31 / 7.32 (9-H / 10-H), 1.97 / 1.94, 1.14 (6-H / 6-H', 7-H), 1.94 / 1.97 , 1.14 (6-H’ / 6-H, 7-H), 1.45 / 1.14 (8-H / 7-H), 1.14 / 1.97, 1.94, 1.45 ( 7-H / 6-H, 6-H’, 8-H). GHSQC (benzene-d $\left.{ }_{6}, 150 / 600 \mathrm{MHz}\right): \delta\left({ }^{13} \mathrm{C}\right) / \delta\left({ }^{1} \mathrm{H}\right)=142.0 / 8.29(\mathrm{o}-\mathrm{C}$ of $\mathrm{Ph} / \mathrm{o}-\mathrm{H}$ of $\mathrm{Ph}), 141.2 / 7.32(\mathrm{C} 10 /$ 10-H), 127.3 / 7.12 (C14' / 14-H'), 127.0 / 7.52 (m-C of Ph / m-H of Ph), 125.4, 125.1 / 6.83 (C14, C15 / 14-H, 15-H), 124.1 / 7.33 (p-C of Ph / p-H of Ph), 122.9 / 6.57 (C3' / 3-H'), 122.7 / 7.14 (C15' / 15-H'), 118.4 / 8.01 (C13' / 13-H'), 116.7 / 7.69 (C13 / 13-H), 114.4 / 5.09 (C4 / 4-H), 114.0 / 5.13 (C4' / 4-H'), 109.8 / 6.97 (C16' / 16-H'), 108.9 / 6.16 (C5 / 5-H), 108.8 / 5.55 (C2 / 2-H), 108.5 / 6.08 (C5' / 5-H'), 108.4 / 5.84 (C2' / 2-H'), 110.9 / 6.46 (C16 / 16-H), 33.3 / 1.97, 1.94 (C6 / 6-H, 6-H'), 32.6 / 3.37 (C9' /9-H'), 30.8 / 2.31 (C9 /9-H), 28.7 / 1.14 (C7 / 7-H), 21.6 / 1.45 (C8 / 8-H), -4.2 / 0.44 $\left(\mathrm{Si}\left(\mathrm{CH}_{3}\right)_{2} / \mathrm{Si}\left(\mathrm{CH}_{3}\right)_{2}\right),-5.9 / 0.46\left(\mathrm{Si}\left(\mathrm{CH}_{3}\right)_{2} / \mathrm{Si}\left(\mathrm{CH}_{3}\right)_{2}\right) . \mathrm{GHMBC}$ (benzene-d, $\left.150 / 600 \mathrm{MHz}\right): \delta\left({ }^{13} \mathrm{C}\right) /$ $\delta\left({ }^{1} \mathrm{H}\right)=202.9 / 3.37\left(\mathrm{C} 10^{\prime} /\right.$ 9-H'), $181.4 / 8.29,7.52($ ipso-C of $\mathrm{Ph} / \mathrm{o}-, \mathrm{m}-\mathrm{H}$ of $\mathrm{Ph}), 143.6 / 8.01$, 7.14, 3.37 (C17' / 13-H', 15-H', 9-H'), 142.0 / 8.29, 7.33 (o-C of Ph / o-, p-H of Ph), 141.2 / 2.31 (C10 / 9-H), 140.8 / 7.12, 6.97 (C12' / 14-H', 16-H'), 140.6 / 6.16, 5.09, 1.97, 1.94 (C3 / 5-H, 4-H, 6-H, 6H'), 136.4 / 7.32, 6.83, 6.46 (C12 / 10-H, 14-H, 15-H, 16-H), 132.7 / 7.32, 6.83, 2.31 (C12 / 10-H, 14H, 15-H, 9-H), 127.3 / 7.14 (C14' / 15-H'), 127.0 / 7.52 (m-C of Ph / m-H of Ph), 125.4 / 6.46 (C14 / 16-H), 125.1 / 7.69 (C15 / 13-H), 124.1 / 8.29 (p-C of Ph / o-H of Ph), 122.9 / 6.08 (C3' / 5-H'), 122.7 / 8.01 (C15' / 13-H'), 118.4 / 7.14, 7.12 (C13' / 15-H', 14-H'), 116.7 / 6.83 (C13 / 14-H, 15-H), 114.4 / 6.16, 5.55, 1.94 (C4 / 5-H, 2-H, 6-H'), 114.0 / 6.08 (C4' / 5-H'), 110.9 / 7.32, 6.83 (C16 / 10-H, $14-$ H, 15-H), 109.8 / 7.12 (C16' / 14-H'), 108.9 / 5.55, 5.09, 1.97 (C5 / 2-H, 4-H ,6-H), 108.8 / 6.16, 5.09 (C2 / 5-H, 4-H), 108.4 / 6.08 (C2' / 5-H'), 102.9 / 6.16, 5.55, 5.09, 0.46, 0.44 (C1 / 5-H, 2-H, 4-H, $\left.\mathrm{Si}\left(\mathrm{CH}_{3}\right)_{2}\right), 102.8$ / 6.08, 6.57, 5.84, 0.46, 0.44 (C1' / 5-H', 3-H', 2-H', $\left.\mathrm{Si}\left(\mathrm{CH}_{3}\right)_{2}\right), 33.3$ / 1.45 (C6 / 8-H), 30.8 / 7.32 (C9 / 10-H), 28.7 / 1.97, 1.94 (C7 / 6-H, 6-H'), 21.6 / 1.97, 1.94 (C8 / 6-H, 6-H'), -4.2 / $0.46\left(\mathrm{Si}\left(\mathrm{CH}_{3}\right)_{2} / \mathrm{Si}\left(\mathrm{CH}_{3}\right)_{2}\right),-5.9 / 0.44\left(\mathrm{Si}\left(\mathrm{CH}_{3}\right)_{2} / \mathrm{Si}\left(\mathrm{CH}_{3}\right)_{2}\right) .{ }^{19} \mathrm{~F}_{-}{ }^{19} \mathrm{~F}$ GCOSY (benzene-d 6 , $564 / 564$ $\mathrm{MHz}): \delta\left({ }^{19} \mathrm{~F}\right) / \delta\left({ }^{19} \mathrm{~F}\right)=-132.9 /-164.0(\mathrm{o}-\mathrm{F} / \mathrm{m}-\mathrm{F}),-133.2 /-164.1\left(\mathrm{o}-\mathrm{F}^{\prime} / \mathrm{m}-\mathrm{F}^{\prime}\right),-158.7 /-164.0(\mathrm{p}-$ F / m-F), -158.8 / -164.1 (p-F' / m-F'), -164.0 / -132.9, -158.7 (m-F / o-F, p-F), -164.1 / -133.2, $158.8\left(\mathrm{~m}-\mathrm{F}^{\prime} / \mathrm{o}-\mathrm{F}^{\prime}, \mathrm{p}-\mathrm{F}^{\prime}\right)$. 
Diastereoisomer 20B: ${ }^{1} \mathrm{H}-\mathrm{NMR}$ (benzene- $\mathrm{d}_{6}, 600 \mathrm{MHz}$ ): $\delta=8.30,7.52,7.33$ (each m, 5H, o-, m-, p-

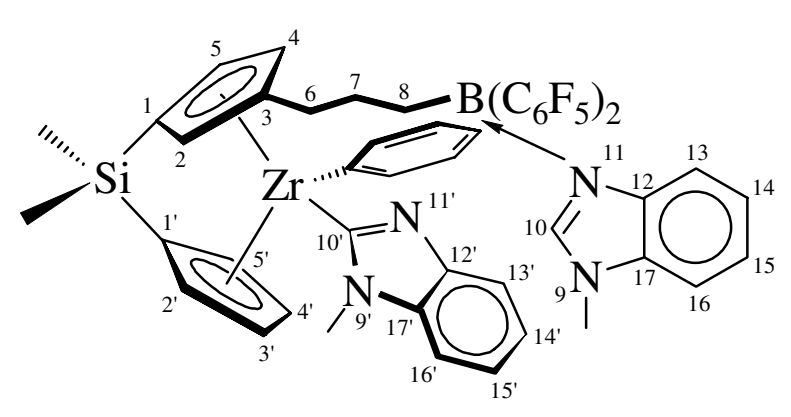

$\mathrm{H}$ of $\mathrm{Ph}), 8.01\left(\mathrm{~m}, 1 \mathrm{H}, 13-\mathrm{H}^{\prime}\right), 7.63(\mathrm{~m}, 1 \mathrm{H}, 13-\mathrm{H})$, $7.19(\mathrm{~s}, 1 \mathrm{H}, 10-\mathrm{H}), 7.14\left(\mathrm{~m}, 1 \mathrm{H}, 15-\mathrm{H}^{\prime}\right), 7.12(\mathrm{~m}, 1 \mathrm{H}$ 14-H'), 6.97 (m, 1H, 16-H'), $6.83(\mathrm{~m}, 2 \mathrm{H}, 14-\mathrm{H}$, 15-H), 6.48 (m, 1H, 4-H'), $6.46(\mathrm{~m}, 1 \mathrm{H}, 16-\mathrm{H}), 6.35$ (m, 1H, 4-H), $6.14\left(\mathrm{~m}, 1 \mathrm{H}, 2-\mathrm{H}^{\prime}\right), 6.09(\mathrm{~m}, 1 \mathrm{H}, 2-\mathrm{H})$ $5.83\left(\mathrm{~m}, 1 \mathrm{H}, 5-\mathrm{H}^{\prime}\right), 5.74(\mathrm{~m}, 1 \mathrm{H}, 5-\mathrm{H}), 5.17\left(\mathrm{~m}, 1 \mathrm{H}, 3-\mathrm{H}^{\prime}\right), 3.43\left(\mathrm{~s}, 3 \mathrm{H}, 9-\mathrm{H}^{\prime}\right), 2.29$ (s, 3H, 9-H), 1.55/1.15 (each m, each 1H, 6-H, 6-H'), 1.40/1.28 (each m, each 1H, 8-H, 8-H'), 0.90 (m, 2H, 7-H), 0.44/0.42 (each s, each 3H, $\left.\operatorname{Si}\left(\mathrm{CH}_{3}\right)_{2}\right) .{ }^{13} \mathrm{C}\left\{{ }^{1} \mathrm{H}\right\}-\mathrm{NMR}$ (benzene-d $6,150 \mathrm{MHz}$ ): $\delta=177.2,141.7,126.8$ 124.1 (ipso-, o-, m-, p-C of Ph), 202.7 (C10’), 143.6 (C17’), 141.2 (C10), 140.8 (C12'), 136.4 (C12), 132.7 (C17), 132.0 (C3), 127.3 (C14'), 125.4 (C14), 125.1 (C15), 124.5 (C4'), 124.4 (C4), 122.7 (C15'), 118.4 (C13’), 116.5 (C13), 112.9 (C3’), 110.9 (C16), 110.8 (C2), 109.8 (C16'), 109.1 (C2'), 107.0 (C5'), 106.9 (C5), 103.5 (C1'), 102.6 (C1), 32.6 (C9'), 31.6 (C6), 30.8 (C9), 28.1 (C7), 20.9 (C8), -4.2/-6.1 $\left(\mathrm{Si}\left(\mathrm{CH}_{3}\right)_{2}\right) ; 148.5\left({ }^{1} \mathrm{~J}_{\mathrm{CF}}=238.4 \mathrm{~Hz}, \mathrm{o}-\mathrm{C}\right.$ of $\left.\mathrm{C}_{6} \mathrm{~F}_{5}\right), 139.4\left({ }^{1} \mathrm{~J}_{\mathrm{CF}}=251.5 \mathrm{~Hz}, \mathrm{p}-\mathrm{C}\right.$ of $\left.\mathrm{C}_{6} \mathrm{~F}_{5}\right)$, $137.5\left({ }^{1} \mathrm{~J}_{\mathrm{CF}}=249.6 \mathrm{~Hz}, \mathrm{~m}-\mathrm{C}\right.$ of $\left.\mathrm{C}_{6} \mathrm{~F}_{5}\right), 121.7$ (ipso-C of $\left.\mathrm{C}_{6} \mathrm{~F}_{5}\right) .{ }^{11} \mathrm{~B}\left\{{ }^{1} \mathrm{H}\right\}-\mathrm{NMR}$ (benzene-d $6,64 \mathrm{MHz}$ ): $\delta=$ $-5.10\left(v_{1 / 2}=341 \mathrm{~Hz}\right) .{ }^{19} \mathrm{~F}-\mathrm{NMR}\left(\right.$ benzene-d $\left._{6}, 564 \mathrm{MHz}\right): \delta=-132.9\left(\mathrm{~m}, 2 \mathrm{~F}, \mathrm{o}-\mathrm{F}\right.$ of $\left.\mathrm{C}_{6} \mathrm{~F}_{5}\right),-133.4(\mathrm{~m}$, $2 \mathrm{~F}, \mathrm{o}-\mathrm{F}^{\prime}$ of $\left.\mathrm{C}_{6} \mathrm{~F}_{5}\right),-158.6\left(\mathrm{t},{ }^{3} \mathrm{~J}_{\mathrm{FF}}=20.8 \mathrm{~Hz}, 1 \mathrm{~F}, \mathrm{p}-\mathrm{F}\right.$ of $\left.\mathrm{C}_{6} \mathrm{~F}_{5}\right),-158.9\left(\mathrm{t},{ }^{3} \mathrm{~J}_{\mathrm{FF}}=20.8 \mathrm{~Hz}, 1 \mathrm{~F}, \mathrm{p}-\mathrm{F}^{\prime}\right.$ of $\left.\mathrm{C}_{6} \mathrm{~F}_{5}\right)$, $-163.9\left(\mathrm{~m}, 2 \mathrm{~F}, \mathrm{~m}-\mathrm{F}\right.$ of $\left.\mathrm{C}_{6} \mathrm{~F}_{5}\right),-164.0\left(\mathrm{~m}, 2 \mathrm{~F}, \mathrm{~m}-\mathrm{F}^{\prime}\right.$ of $\left.\mathrm{C}_{6} \mathrm{~F}_{5}\right)$. 1D-TOCSY (benzene-d, $\left.600 / 600 \mathrm{MHz}\right)$ : $\delta\left({ }^{1} \mathrm{H}\right)_{\text {irr. }} / \delta\left({ }^{1} \mathrm{H}\right)_{\text {resp. }}=8.30 / 7.52,7.33(\mathrm{o}-\mathrm{H}$ of $\mathrm{Ph} / \mathrm{m}-, \mathrm{p}-\mathrm{H}$ of $\mathrm{Ph}), 8.01 / 7.14,7.12,6.97\left(13-\mathrm{H}^{\prime} / 15-\right.$ H', 14-H', 16-H'), 7.63 / 6.83, 6.46 (13-H / 14-H, 15-H, 16-H), 5.74 / 6.09, 5.19 (5-H / 2-H, 4-H), 5.17 / 6.48, 5.83, 6.14 (3-H' / 4-H', 5-H', 2-H'), 1.55 / 1.40, 1.28, 1.15, 0.90 (6-H / 8-H, 8-H', 6-H', 7-H). NOE-Diff (benzene-d $6,600 / 600 \mathrm{MHz}): \delta\left({ }^{1} \mathrm{H}\right)_{\text {irr. }} / \delta\left({ }^{1} \mathrm{H}\right)_{\text {resp. }}=8.30 / 8.01,7.52,6.48,6.35,5.83,5.74$ (o-H of Ph / 13-H', m-H of Ph, 4-H', 4-H, 5-H', 5-H), 8.01 / 7.12 (13-H' / 14-H'), 7.52 / 8.30, 7.33 (mH of Ph / o-, p-H of Ph), 6.83 / 7.63, 6.46 (14-H, 15-H / 13-H, 16-H), 6.48 / 5.83, 5.17 (4-H' / 5-H', 3H'), 6.46 / 6.83, 2.29 (16-H / 15-H, 9-H), 6.35 / 5.74, 1.55 (4-H / 5-H, 6-H), 6.14 / 8.30, 5.17, 3.43, 
0.44 (2-H' / o-H of Ph, 3-H', 9-H', $\left.\mathrm{Si}\left(\mathrm{CH}_{3}\right)_{2}\right), 6.09$ / 8.30, 3.43, 0.44 (2-H / o-H of Ph, 9-H', $\left.\mathrm{Si}\left(\mathrm{CH}_{3}\right)_{2}\right)$, 5.74 / 8.30, 6.35, 0.42 (5-H / o-H of Ph, 4-H, Si( $\left.\left(\mathrm{CH}_{3}\right)_{2}\right), 5.83$ / 6.48, $0.42\left(5-\mathrm{H}^{\prime}\right.$ / 4-H', $\left.\mathrm{Si}\left(\mathrm{CH}_{3}\right)_{2}\right), 5.17$ / 8.30, 6.48, 6.14, 6.09 (3-H' / o-H of Ph, 4-H', 2-H', 2-H), 3.43 / 8.30, 8.01, 6.97, 6.14, 6.09, 0.44 (9H' / o-H of Ph, 13-H', 16-H', 2-H', 2-H, Si $\left.\left(\mathrm{CH}_{3}\right)_{2}\right), 1.55$ / 8.30, 8.01, 6.35, 1.15, 0.90 (6-H / o-H of Ph,

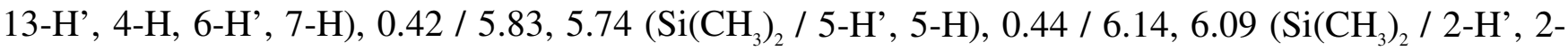
H). GCOSY (benzene-d $\left.{ }_{6}, 600 / 600 \mathrm{MHz}\right): \delta\left({ }^{1} \mathrm{H}\right) / \delta\left({ }^{1} \mathrm{H}\right)=8.30 / 7.52,7.33$ (o-H of Ph / m-H of Ph, pH of Ph), 8.01 / 7.12 (13-H' / 14-H'), 7.63 / 6.83 (13-H / 15-H, 14-H), 7.52 / 8.30, 7.33 (m-H of Ph / o$\mathrm{H}$ of $\mathrm{Ph}, \mathrm{p}-\mathrm{H}$ of $\mathrm{Ph}), 7.33$ / 7.52 (p-H of $\mathrm{Ph} / \mathrm{m}-\mathrm{H}$ of $\mathrm{Ph}), 7.19$ / 2.29 (10-H / 9-H), 7.14 / 6.97 (15-H' / 16-H'), 7.12 / 7.14 (14-H' / 15-H'), 6.97 / 7.14 (16-H' / 15-H’), 6.83 / 7.63, 6.46 (14-H, 15-H / 13-H, 16-H), 6.48 / 5.83, 5.17 (4-H' / 5-H', 3-H'), 6.46 / 6.83 (16-H / 14-H, 15-H), 6.35 / 5.74 (4-H / 5-H), 6.14 / 6.48, 5.83, 5.17 (2-H' / 4-H', 5-H', 3-H'), 6.09 / 6.35, 5.74 (2-H / 4-H, 5-H), 5.83 / 6.48, 6.14, 5.17 (5-H' / 4-H', 2-H’, 3-H’), 5.74 / 6.35, 6.09 (5-H / 4-H, 2-H), 5.17 / 6.48, 6.14, 5.83 (3-H' / 4-H', 2-H', 5-H'), 2.29 / 7.19 (9-H / 10-H), 1.55 / 1.15, 0.90 (6-H / 6-H', 7-H), 1.40 / 1.28, 0.90 (8-H / 8-H', 7-H), 1.28 / 1.40, 0.90 (8-H’ / 8-H, 7-H), 1.15 / 1.55, 0.90 (6-H' / 6-H, 7-H), 0.90 / 1.55, 1.40, 1.28, 1.15 ( 7-H / 8-H, 8-H', 6-H, 6-H'). GHSQC (benzene-d $\left.{ }_{6}, 150 / 600 \mathrm{MHz}\right): \delta\left({ }^{13} \mathrm{C}\right) / \delta\left({ }^{1} \mathrm{H}\right)=141.7 /$ 8.30 (o-C of Ph / o-H of Ph), 141.2 / 7.19 (C10 / 10-H), 127.3 / 7.12 (C14' / 14-H'), 126.8 / 7.52 (m-C of $\mathrm{Ph} / \mathrm{m}-\mathrm{H}$ of Ph), 125.4, 125.1 / 6.83 (C14, C15 / 14-H, 15-H), 124.5 / 6.48 (C4' / 4-H'), 124.4 / 6.35 (C4 / 4-H), 124.1 / 7.33 (p-C of Ph / p-H of Ph), 122.7 / 7.14 (C15' / 15-H'), 118.4 / 8.01 (C13' / 13H'), 116.5 / 7.63 (C13 / 13-H), 112.9 / 5.17 (C3' / 3-H'), 110.9 / 6.46 (C16 / 16-H), 110.8 / 6.09 (C2 / 2-H), 109.8 / 6.97 (C16' / 16-H'), 109.1 / 6.14 (C2' / 2-H'), 107.0 / 5.83 (C5' / 5-H'), 106.9 / 5.74 (C5 / 5-H), 32.6 / 3.43 (C9' / 9-H'), 31.6 / 1.55, 1.15 (C6 / 6-H, 6-H'), 30.8 / 2.29 (C9 /9-H), 28.1 / 0.90

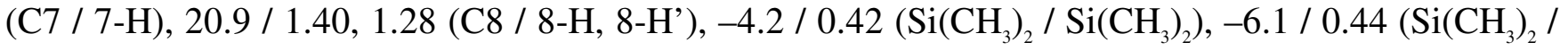
$\left.\operatorname{Si}\left(\mathrm{CH}_{3}\right)_{2}\right)$. GHMBC (benzene-d $\left.6,150 / 600 \mathrm{MHz}\right): \delta\left({ }^{13} \mathrm{C}\right) / \delta\left({ }^{1} \mathrm{H}\right)=202.7 / 3.43\left(\mathrm{C} 10^{\prime} / 9-\mathrm{H}^{\prime}\right), 177.2 /$ 8.30, 7.52 (ipso-C of Ph / o-, m-H of Ph), 143.6 / 8.01, 7.14, 3.43 (C17' / 13-H', 15-H', 9-H'), 141.7 / 8.30, 7.33 (o-C of Ph / o-, p-H of Ph), 141.2 / 2.29 (C10 / 9-H), 140.8 / 7.12, 6.97 (C12' / 14-H', 16H'), 136.4 / 7.19, 6.83, 6.46 (C12 / 10-H, 14-H, 15-H, 16-H), 132.7 / 7.19, 6.83, 2.29 (C12 / 10-H, 14- 
H, 15-H, 9-H), 132.0 / 6.35, 6.09, 5.74, 1.55, 1.15 (C3 / 4-H, 2-H, 5-H, 6-H, 6-H'), 127.3 / 7.14 (C14' / 15-H'), 126.8 / 7.52 (m-C of Ph / m-H of Ph), 125.4 / 6.46 (C14 / 16-H), 124.5 / 6.14, 5.83 (C4' / 2-H', 5-H'), 124.4 / 6.09, 5.74, 1.15 (C4 / 2-H, 5-H, 6-H'), 125.1 / 7.63 (C15 / 13-H), 124.1 / 8.30 (p-C of Ph / o-H of Ph), 122.7 / 8.01 (C15' / 13-H'), 118.4 / 7.14, 7.12 (C13' / 15-H', 14-H'), 116.5 / 6.83 (C13 / 14-H, 15-H), 112.9 / 6.14, 5.83 (C3' / 2-H', 5-H'), 110.9 / 6.83 (C16 / 14-H, 15-H), 110.8 / 6.35, 5.74, 1.55 (C2 / 4-H, 5-H, 6-H), 109.8 / 7.12 (C16' / 14-H'), 109.1 / 6.48, 5.83 (C2' / 4-H', 5-H'), 107.0 / 6.14 (C5' / 2-H'), 106.9 / 6.35, 6.09 (C5 / 4-H, 2-H), 103.5 / 6.14, 5.83, 0.42, 0.44 (C1' / 2-H', 5-H', $\left.\mathrm{Si}\left(\mathrm{CH}_{3}\right)_{2}\right), 102.6$ / 6.35, 6.09, 5.74, 0.42, 0.44 (C1 / 4-H, 2-H, 5-H, Si( $\left.\left.\mathrm{CH}_{3}\right)_{2}\right), 30.8$ / 7.19 (C9 / 10-H), 28.1 / 1.55, 1.40, 1.28, 1.15 (C7 / 6-H, 8-H, 8-H', 6-H'), 20.9 / 1.55, 1.28 (C8 / 6-H, 8-H'), -4.2 / 0.44 $\left.\left(\mathrm{Si}_{\left(\mathrm{CH}_{3}\right.}\right)_{2} / \mathrm{Si}\left(\mathrm{CH}_{3}\right)_{2}\right),-6.1 / 0.42\left(\mathrm{Si}\left(\mathrm{CH}_{3}\right)_{2} / \mathrm{Si}\left(\mathrm{CH}_{3}\right)_{2}\right) .{ }^{19} \mathrm{~F} /{ }^{19} \mathrm{~F} \mathrm{GCOSY}($ benzene-d 6 , $564 / 564 \mathrm{MHz}): \delta$ $\left({ }^{19} \mathrm{~F}\right) / \delta\left({ }^{19} \mathrm{~F}\right)=-132.9 /-163.9(\mathrm{o}-\mathrm{F} / \mathrm{m}-\mathrm{F}), \quad-133.4 /-164.0\left(\mathrm{o}-\mathrm{F}^{\prime} / \mathrm{m}-\mathrm{F}\right),-158.6 /-163.9(\mathrm{p}-\mathrm{F} /$ m-F), -158.9 / -164.0 (p-F' / m-F'), -163.9 / -132.9, -158.6 (m-F / o-F, p-F), -164.0 / -133.4, -158.9 (m-F'/ o-F', p-F'). 